Universidade de São Paulo

Instituto de Astronomia, Geofísica e Ciências Atmosféricas

Departamento de Astronomia

Viviane Salvador Alves

\title{
Calibração de metalicidades de estrelas
} subanãs $M$ pobres em metais baseada em companheiras binárias 



\section{Calibração de metalicidades de estrelas subanãs $M$ pobres em metais baseada em companheiras binárias}

Dissertação apresentada ao Departamento de Astronomia do Instituto de Astronomia, Geofísica e Ciências Atmosféricas da Universidade de São Paulo como requisito parcial para a obtenção do título de Mestre em Ciências.

"Versão Corrigida. O original encontra-se disponível na Unidade."

Área de Concentração: Astronomia Orientadora: Prof ${ }^{\mathrm{a}}$. Dra ${ }^{\mathrm{a}}$. Silvia Rossi 

Dedico este trabalho aos meus pais pelo apoio incondicional. 



\section{Agradecimentos}

Agradeço à minha família por todo apoio e compreensão.

Ao meu namorado, Leandro, pela ajuda que me prestou na execução deste trabalho, pela paciência e pelo carinho.

À minha orientadora, Silvia Rossi, pela oportunidade de enriquecer meus conhecimentos. Ao pesquisador Vinicius Placco por colaborar em muito na realização deste trabalho.

Ao professor Dr. Jorge Meléndez pelos conselhos valiosos.

Ao pessoal da seção de pós-graduação e secretaria por sempre serem prestativos e atenciosos.

E à FAPESP, pelo apoio financeiro, sob o projeto no: 2011/03658-1.

À Capes e ao CNPq, pelo apoio financeiro ao programa de pós-graduação.

Esta dissertação foi escrita em IATEX com a classe IAGTESE, para teses e dissertações do IAG. 

Deve seguir seu caminho, trabalhando além dos limites da esperança e talvez além dos limites da razão. Talvez tenha de continuar sem trégua, mesmo através do Grande Deserto Experimental, mas por fim chegará a seu destino. Robert Gilmore - O mágico dos quarks 



\section{Resumo}

Este trabalho envolve um estudo espectroscópico voltado para estimativas de metalicidades de uma amostra de estrelas anãs $M$. As estrelas $M$ de baixa massa constituem os objetos estelares mais numerosos na Galáxia e com tempos de vida de sequência principal que excedem a atual idade do Universo. Sendo assim, podem ser vistos como grandes laboratórios para estudo da estrutura e evolução da Galáxia. Os esforços deste trabalho se concentraram em traçar paralelos entre a força de algumas bandas moleculares presentes nos espectros dessas estrelas e a metalicidade. Seguiu-se para isso metodologias presentes na literatura. A motivação para o trabalho foi dar continuidade a um estudo iniciado por Sebastien Lépine e colaboradores, em 2007, utilizando, pela primeira vez uma amostra de anãs $\mathrm{M}$ do hemisfério sul. Além disso, uma reavaliação da calibração de metalicidade para anãs M norteou o estudo.

O trabalho utilizou o espectrógrafo Goodman do observatório SOAR para obtenção dos espectros estelares. As estimativas de metalicidade da amostra foram obtidas a partir do n-sspp, uma adaptação do Segue Stellar Parameters Pipeline (sspp), pipeline do SDSS. Os objetos considerados são sistemas binários constituídos por uma estrela de tipo espectral F ou G e outra de tipo espectral M. A estrela primária ( F ou G) é utilizada para se determinar a metalicidade da estrela M (secundária), diante da hipótese de que o sistema se formou a partir de uma mesma nuvem mãe.

Apesar de todos esforços empregados neste estudo, não foi possível refinar a calibração de metalicidades para as estrelas em questão. O índice calibrador desenvolvido por Lépine et al. (2007) mostrou-se um fraco indicador de metalicidades, resultado já documentado por eles em 2012. Com isso, os estudos para calibrações de metalicidade de estrelas anãs M devem ser incentivados. 



\section{Abstract}

The aim of this work is to obtain metallicity calibration for a sample of $\mathrm{M}$ dwarfs stars through spectroscopic studies. The low-mass M stars are the most numerous objects in the Galaxy and their main sequence lifetimes exceed the current age of the Universe. Thus, they reveal themselves as large laboratories for studying the structure and evolution of the Galaxy. The efforts of this work are focused in the construction of relations between the strengths of some molecular bands and metallicities for M stars. For that, methodologies in the literature were followed. The motivation of this work was to continue a study initiated by Lépine et al. (2007) using for the first time a sample of M dwarf from southern hemisphere. Besides that, there is yet no definitive metallicity calibration for M dwarfs.

Goodman spectrograph at SOAR was used to obtain the stellar spectra. Metallicities estimates for the sample were obtained from an adapted version of the Segue Stellar Parameters Pipeline (sspp), the SDSS's pipeline. The studied objects are binary systems consisting of a F or G spectral type star and a M stars. The primary star (F or G) is used to estimate the metallicity of the M star (secondary), since it is assumed that the system is formed from the same parent cloud.

Despite all efforts made in this work, it was not possible to refine the metallicities calibration for the analyzed sample. The index originally developed by Lépine et al. (2007) proved to be a poor metallicity indicator, as already documented by them in 2012. Thus, metallicity calibration studies for M dwarf stars should be encouraged. 



\section{Lista de Figuras}

1.1 Diagrama HR mostrando a localização das estrelas subanãs . . . . . . . . . .

1.2 Espectros de subanãs frias pobres em metais observadas por S. Lépine no telescópio de 4-m Mayall. Todos os espectros estão normalizados a 1 em $7500 \AA$, e deslocados verticalmente em passos de 1 unidade para clareza. O painel esquerdo mostra a sequência de subanãs M (sdM), o do meio apresenta as subanãs $M$ extremas (esdM) e o painel direito as subanãs ultra pobres em metais (usdM), segundo a classificação de Lépine et al. (2007) (seção 1.3.3). As duas bandas dominantes, fáceis de serem medidas, de CaH eTiO estão identificadas no espectro esquerdo inferior. As bandas são claramente mais fortes nos objetos mais tardios (mais frios). Note-se também como a razão TiO/CaH decresce com a metalicidade. . . . . . . . . . . . . . . . . . .

1.3 Diagrama de movimento próprio reduzido construido por Lépine et al. (2007) para anãs M e subanãs M mostrando a separação entre estrelas do disco e do halo galáctico . . . . . . . . . . . . . . . . . .

2.1 Estrela PM_I00426-2315 com indicação de algumas linhas atômicas. Configuração azul. Note que a ordenada está em unidades de contagens. . . . . 38

2.2 Estrela PM_I00426-2314 com indicação de algumas bandas moleculares. Configuração vermelha. Note que a ordenada é apresentada em densidade de fluxo. . . . . . . . . . . . . . . . . . .

$3.1[\mathrm{CaH} 2]+[\mathrm{CaH} 3]$ versus $[\mathrm{TiO} 5]$ para estrelas do disco e do halo para a amostra de Lépine et al. (2007) . . . . . . . . . . . . . . . . . . . 
$3.2[\mathrm{CaH} 2]+[\mathrm{CaH} 3]$ versus [TiO5] do trabalho de Woolf et al. 2009. Números próximos aos pontos indicam estrelas com seus respectivos valores de $[\mathrm{Fe} / \mathrm{H}]$. Círculos cheios são estrelas do trabalho de Woolf \& Wallerstein (2006) e quadrados cheios são estrelas do trabalho de Woolf et al. (2009) . . . . . . .

3.3 Comparação entre o índice de metalicidade $\zeta_{T i O / C a H}$ para componentes de sistemas binários resolvidos (de Lépine et al. 2007) . . . . . . . . . . . . .

3.4 Classificação de subanãs em subclasses de metalicidade de acordo com G97. sdM aparecem como quadrados cheios, esdM como triângulos cheios, sdK como círculos cheios e estrelas não classificadas como subanãs aparecem como círculos abertos. A barra de erro de \pm 0.03 aparece no canto inferior direito. . . . . . . . . . . . . . . . . . . . .

3.5 Distribuição dos valores dos índices de $\mathrm{TiO} 5$ e $\mathrm{CaH} 2+\mathrm{CaH} 3$ para pares de movimento próprio comum. As primárias e secundárias são conectadas por linhas retas. No antigo sistema de classificação (esquerda), alguns pares atravessavam os separadores de subclasses de forma que os membros do sistema apresentavam diferentes metalicidades. O novo sistema (direita) (Lépine et al. 2007) assegura que primária e secundária se localizem na mesma subclasse, que é o que se espera de pares que tiveram a mesma origem. . . . .

3.6 Calibração de Woolf et al. (2009) para uma amostra de sdM e esdM observada em alta resolução. Os pontos são as estrelas e a linha sólida o ajuste linear. $[\mathrm{Fe} / \mathrm{H}]=a+b \zeta_{\mathrm{TiO} / \mathrm{CaH}} \operatorname{com} a=-1.685 \pm 0.079$ e $b=1.632 \pm 0.096$.

4.1 Comparação entre este trabalho e o de Woolf et al. (2009) para a relação entre [CaH2]+[CaH3] e [CaH2]. A relação obtida por Woolf et al. (2009) foi $[\mathrm{CaH} 2]=(-0.282 \pm 0.014)+(0.635 \pm 0.010)([\mathrm{CaH} 2]+[\mathrm{CaH} 3])$ e a obtida neste trabalho foi $[\mathrm{CaH} 2]=(-0.261 \pm 0.020)+(0.619 \pm 0.015)([\mathrm{CaH} 2]+$

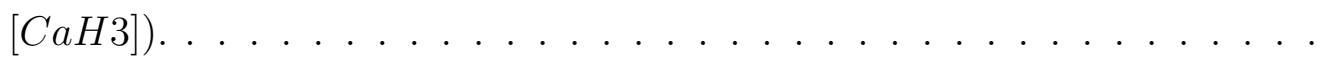

4.2 Efeito da calibração em fluxo: (a) Comparação entre o índice de TiO5 calculado com e sem calibração em fluxo. (b) Comparação entre a soma dos índices de $\mathrm{CaH} 2+\mathrm{CaH} 3$ calculado com e sem calibração em fluxo . . . . . 58

4.3 Exemplo de espectro com correção de franjas eficiente. . . . . . . . . . . 61

4.4 Exemplo de espectro com correção de franjas ineficiente. . . . . . . . . . . 61 
4.5 Efeito da correção de franjas: (a) Comparação entre o índice de TiO5 calculado com e sem correção de franjas. (b) Comparação entre a soma dos índices de $\mathrm{CaH} 2+\mathrm{CaH} 3$ calculado com e sem correção de franjas. . . . . . . 63

4.6 Variação nas bandas de $\mathrm{CaH}$ e TiO5 com a mudança dos subtipos espectrais 65

4.7 Diagrama $[\mathrm{CaH} 2]+[\mathrm{CaH} 3]$ versus $[\mathrm{TiO} 5]$ mostrando a localização da nossa amostra de anãs M (figura da esquerda) e as respectivas metalicidades delas (figura da direita).

$4.8[\mathrm{Fe} / \mathrm{H}]$ versus $\mathrm{K}_{\text {index }}$ : (a) Amostra de sistemas com anãs M com S/N>20. (b) Amostra com todos os sistemas observados. . . . . . . . . . . . 68

$4.9[\mathrm{Fe} / \mathrm{H}]$ versus $\zeta_{T i O / C a H}$ utilizando a equação para $[\mathrm{TiO} 5]_{Z_{\odot}}$ de Lépine et al. (2007)

$4.10[\mathrm{Fe} / \mathrm{H}]$ versus $\zeta_{T i O / C a H}$ utilizando a equação para $[\mathrm{TiO} 5]_{\odot}$ de Dithal et al. (2012)

$4.11[\mathrm{Fe} / \mathrm{H}]$ versus $\zeta_{T i O / C a H}$ utilizando a equação para $[\mathrm{TiO} 5]_{Z_{\odot}}$ de Lépine et al. (2012)

$4.12[\mathrm{Fe} / \mathrm{H}]$ versus $\zeta_{T i O / C a H}$ utilizando a equação para $[\mathrm{TiO} 5]_{Z_{\odot}}$ de Dithal et al. (2012). A reta em rosa mostra o ajuste para toda amostra válida: $[\mathrm{Fe} / \mathrm{H}]=$ $(-1.6 \pm 0.7)+(0.88 \pm 0.78) \zeta_{\mathrm{TiO} / \mathrm{CaH}}$. A reta em azul mostra o ajuste excluindo a estrela PM_02300-2402E: $[\mathrm{Fe} / \mathrm{H}]=(-1.9 \pm 0.7)+(1.2 \pm 0.7) \zeta_{\mathrm{TiO} / \mathrm{CaH}} . \mathrm{A}$ reta em verde mostra a calibração de Woolf et al. (2009). . . . . . . . . . .

4.13 Comparação entre $\zeta_{T i O / C a H}$ e metalicidades obtidas por métodos independentes para uma amostra de anãs M feita por Lépine et al. (2012). O painel de cima compara $\zeta_{T i O / C a H} \mathrm{com}[\mathrm{Fe} / \mathrm{H}]$ obtido através da calibração fotométrica de Neves et al. (2012). O painel de baixo compara $\zeta_{\mathrm{TiO} / \mathrm{CaH}} \mathrm{com}$ [Fe/H] obtido pela calibração no infravermelho de Rojas-Ayala (2010). . . .

4.14 Comparação feita por Lépine et al. (2012) entre índices espectrais medidos a partir de três diferentes observatórios (MDM, Palomar-MSU e UH), mostrando a dependência instrumental desses índices. . . . . . . . . . . . .

4.15 Teste feito por Lépine et al. (2012) do $\zeta_{T i O / C a H}$ em função do subtipo espectral mostrando que a imprecisão na medição de $\zeta_{T i O / C a H}$ aumenta para subtipos mais jovens. . . . . . . . . . . . . . . . . . . 74

$4.16[\mathrm{C} / \mathrm{Fe}]$ versus $[\mathrm{Fe} / \mathrm{H}]$ para a amostra de anãs M. . . . . . . . . . . . . 75 
$4.17[\mathrm{C} / \mathrm{Fe}]$ versus $\mathrm{K}_{\text {index }}$ : (a) $\zeta_{\text {TiO/CaH }}$ em acordo com Dithal et al. (2012). (b) $\zeta_{\mathrm{TiO} / \mathrm{CaH}}$ em acordo com Lépine et al. (2012). . . . . . . . . . . 76

B.1 $\operatorname{Par} 1 \ldots \ldots \ldots \ldots \ldots \ldots$

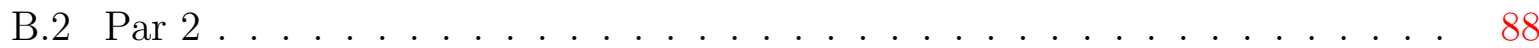

B.3 Par $3 \ldots \ldots \ldots \ldots \ldots \ldots$

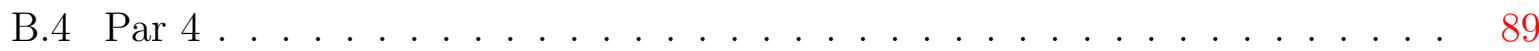

B.5 Par $5 \ldots \ldots \ldots \ldots \ldots \ldots \ldots$

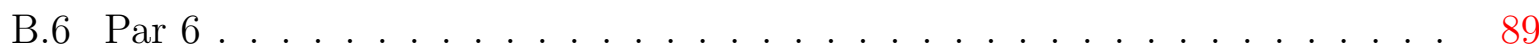

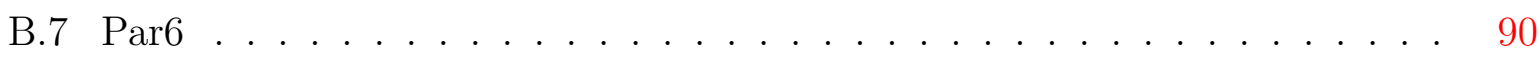

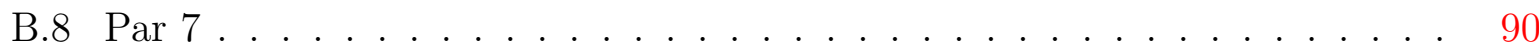

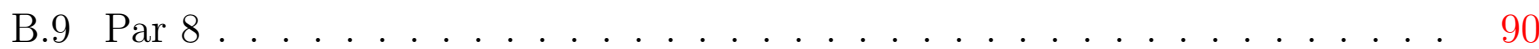

B.10 Par $9 \ldots \ldots \ldots \ldots \ldots$. . . . . . . . . . . . . . . . . . . . .

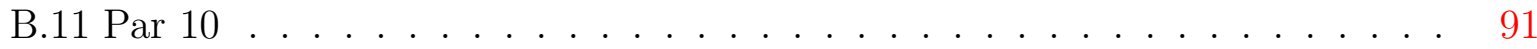

B.12 $\operatorname{Par} 11 \ldots \ldots \ldots \ldots \ldots \ldots$

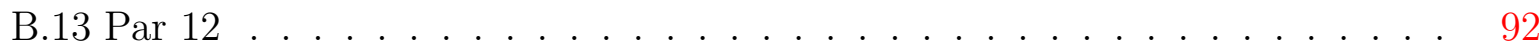

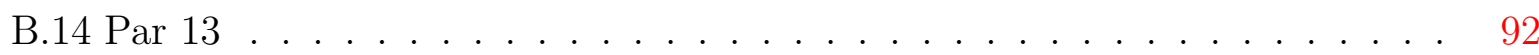

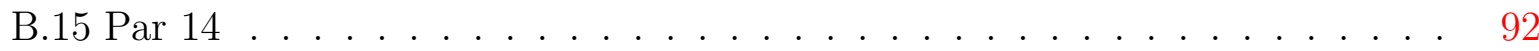

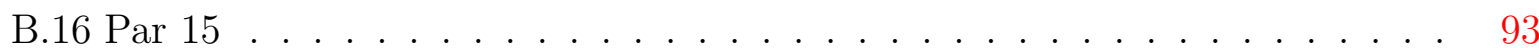

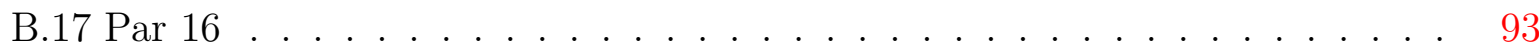

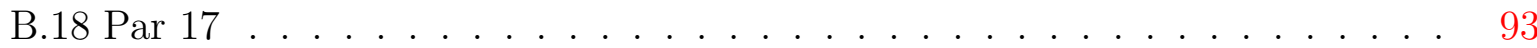

B.19 Par $18 \ldots \ldots \ldots \ldots . \ldots \ldots . \ldots \ldots$

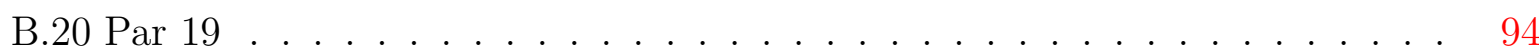

B.21 Par $19 \ldots \ldots \ldots \ldots . \ldots \ldots \ldots$

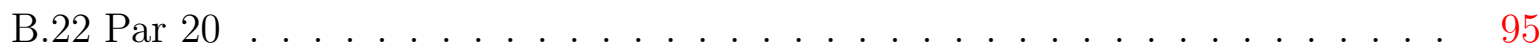

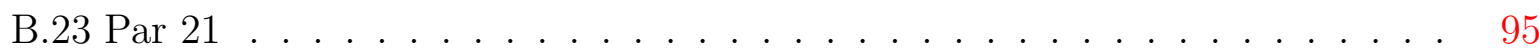

B.24 Par $22 \ldots \ldots \ldots \ldots \ldots \ldots$

B.25 Par $23 \ldots \ldots \ldots \ldots \ldots \ldots$

B.26 Par $24 \ldots \ldots \ldots \ldots \ldots \ldots$

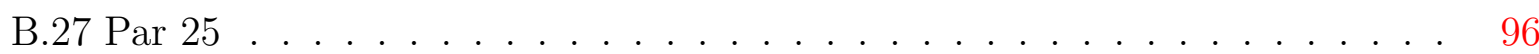

B.28 Par $26 \ldots \ldots \ldots \ldots \ldots \ldots \ldots \ldots$

B.29 Par $27 \ldots \ldots \ldots \ldots \ldots$. . . . . . . . . . . . . . . . . . . . . . . . . . 


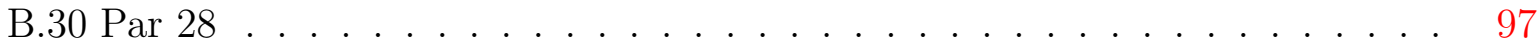

B.31 Par $36 \ldots \ldots \ldots \ldots \ldots$

B.32 Par $37 \ldots \ldots \ldots \ldots \ldots \ldots$

B.33 Par $38 \ldots \ldots \ldots \ldots \ldots \ldots$

B.34 Par $39 \ldots \ldots \ldots \ldots$

B.35 Par $40 \ldots \ldots \ldots \ldots \ldots \ldots$ 



\section{Lista de Tabelas}

2.1 Posições (J2000) e movimentos próprios para todas as estrelas observadas extraídas do catálogo LSPM-south. A terceira coluna indica se a estrela está presente no catálogo Hipparcos. A coluna 13 indica quais estrelas secundárias apresentam razão $\mathrm{S} / \mathrm{N}>20$ e as duas últimas colunas indicam o $K_{\text {index }}$ e a extinção EBVA para o sistema, respectivamente. Objetos em vermelho indicam sistemas triplos e em azul os pares removidos com a atualização do catálogo. . . . . . . . . . . . . . . . . . . . .

2.2 Fotometria para todas as estrelas observadas extraídas do catálogo LSPMsouth. A terceira coluna indica se a estrela está presente no catálogo Hipparcos. A coluna 13 indica quais estrelas secundárias apresentam razão $\mathrm{S} / \mathrm{N}>20$ e as duas últimas colunas indicam o $K_{\text {index }}$ e a extinção EBVA para o sistema, respectivamente. Objetos em vermelho indicam sistemas triplos e em azul os pares removidos com a atualização do catálogo. . . . . 40

2.3 Número de estrelas observadas em cada noite ao longo de 3 semestres. . . . 41

2.4 Estrelas padrões espectrofotométricas observadas para calibração em fluxo. 41

3.1 Índices espectrais originalmente definidos por Reid et al. (1995). Os índices são calculados a partir da razão do fluxo médio ao longo dos intervalos de comprimento de onda especificados. . . . . . . . . . . . . . .

3.2 Divisão em subclasses de metalicidades como apresentada em Woolf et al.(2009) e em acordo com as quatro subclasses propostas por Lépine et

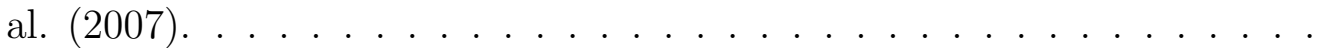


4.1 Índices espectroscópicos e metalicidades para as anãs M. A incerteza nas abundâncias é de 0.2 dex. . . . . . . . . . . . . . . . . 56

4.2 Comparação entre os índices espectroscópicos obtidos com e sem calibração em fluxo. . . . . . . . . . . . . . . . . . . . . . . 59

4.3 Comparação entre os índices espectroscópicos obtidos com e sem correção de franjas. . . . . . . . . . . . . . . . . . . . 62

4.4 Subtipos espectrais das estrelas secundárias da amostra desse trabalho de acordo com as calibrações de G97. As determinações foram feitas utilizando

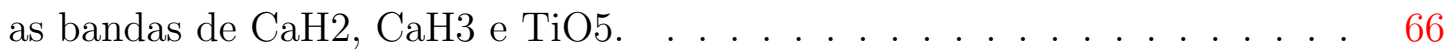

A.1 Resultados para $[\mathrm{Fe} / \mathrm{H}]$ e $[\mathrm{C} / \mathrm{Fe}]$ obtido pelo n-sspp para todos os pares observados. O incerteza nas abundâncias é de 0.2 dex $\ldots . . . .86$ 


\section{Sumário}

1. Introdução . . . . . . . . . . . . . . . . . . . . . . . . . . 23

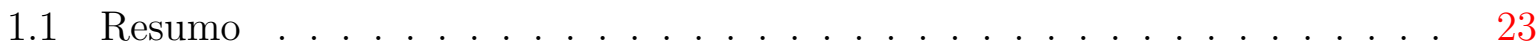

1.2 Estrelas de tipo espectral $\mathrm{M} \ldots \ldots \ldots$. . . . . . . . . . . . 24

1.2.1 Classificação de temperatura . . . . . . . . . . . . . . 25

1.2.2 Anãs versus Gigantes e o $\log (\mathrm{g})$. . . . . . . . . . . . . . . . 25

1.3 Estrelas subanãs $\mathrm{M} \ldots \ldots \ldots$. . . . . . . . . . . . . . . . . 26

1.3.1 Relação metalicidade/raio . . . . . . . . . . . . . . . . . 27

1.3.2 Características espectroscópicas . . . . . . . . . . . . . . . . 27

1.3.3 Classificação de metalicidade . . . . . . . . . . . . . 28

1.3.4 Como separar anãs do disco de subanãs do halo . . . . . . . . . . . 30

1.4 Sistemas binários: Detecção . . . . . . . . . . . . . . . . . . . . . 31

1.4.1 Subanãs em sistemas binários . . . . . . . . . . . . . . . . . . 32

1.5 Este trabalho . . . . . . . . . . . . . . . . . . . . . . . 32

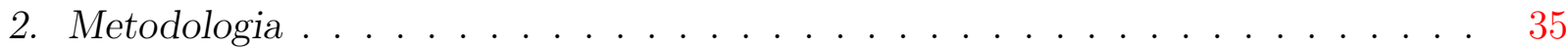

2.1 O catálogo e a seleção . . . . . . . . . . . . . . . . . . . . 35

2.2 Observações e redução dos dados . . . . . . . . . . . . . . . . 36

2.3 Como os dados foram obtidos . . . . . . . . . . . . . . . . 41

3. Calibrações de metalicidades . . . . . . . . . . . . . . . . . . . . . . 43

3.1 Calibrações fotométricas . . . . . . . . . . . . . . . . . . . . . . . 43

3.2 Calibrações espectroscópicas . . . . . . . . . . . . . . . . . 44

3.2.1 Força das bandas moleculares . . . . . . . . . . . . . 44

$3.2 .2 \quad$ O índice de metalicidade $\zeta_{\mathrm{TiO} / \mathrm{CaH}} \ldots \ldots \ldots \ldots . \ldots . \ldots . \ldots$ 
3.2.3 Subclasses de metalicidade . . . . . . . . . . . . . . . . . . 48

4. Resultados e discussões . . . . . . . . . . . . . . . . . . . . . . . 55

4.1 Validação dos índices espectroscópicos .......... . 57

4.2 A calibração em fluxo é necessária? . . . . . . . . . . . . . . . . . . . 57

4.3 Efeito das franjas no cálculo dos índices espectroscópicos . . . . . . . . . . 60

4.4 Os sistemas triplos . . . . . . . . . . . . . . . . . . 63

4.5 Subtipos espectrais . . . . . . . . . . . . . . . . . 64

4.6 Comparação com outros trabalhos . . . . . . . . . . . . . . . . 66

4.6.1 Diagrama $[\mathrm{CaH} 2]+[\mathrm{CaH} 3]$ versus $[\mathrm{TiO} 5] \ldots \ldots . \ldots . \ldots 66$

$4.6 .2 \mathrm{~K}_{\text {index }}$ e a metalicidade . . . . . . . . . . . . . . . 67

4.6.3 $\zeta_{\mathrm{TiO} / \mathrm{CaH}}$ como indicador de metalicidade . . . . . . . . . 68

4.7 Carbonicidade . . . . . . . . . . . . . . . . . 75

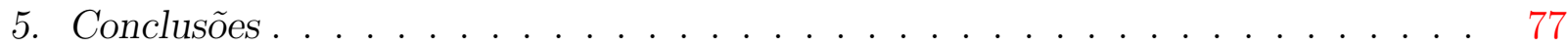

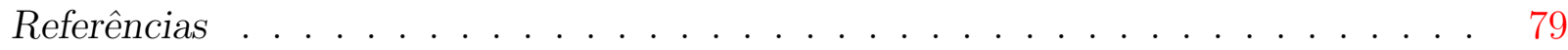

Apêndice $\quad 83$

A. Resultados para toda amostra observada . . . . . . . . . . . . . . . . 85

B. Espectros estelares . . . . . . . . . . . . . . . . . . . 87 
Capítulo 1

\section{Introdução}

\subsection{Resumo}

Pode-se dizer que na nossa Galáxia, nem todas as estrelas tem a mesma composição química. Tal constatação sugere épocas, locais e condições físicas distintas para uma classe de objetos. Em particular, encontra-se estrelas cujas abundâncias atmosféricas de elementos pesados (principalmente o Fe) são substancialmente menores que seu equivalente $\operatorname{solar}\left(Z_{\odot}=0.02\right)$.

Esses objetos são chamados pobres em metal e podem fornecer importantes evidências sobre o início da Galáxia, bem como do Universo. Porém, o termo "pobre em metal" não necessariamente reflete o conteúdo metálico total da estrela, e sim a abundância do elemento químico ferro (metalicidade), o qual não é o mais abundante, mas o mais fácil de ser medido com dados espectrais no visível.

A metalicidade de um objeto fornece uma indicação de sua idade. Quando o Universo se formou, de acordo com a teoria do Big Bang, ele consistia quase totalmente de hidrogênio, o qual via nuclessíntese primordial, criou uma quantidade significativa de hélio e traços de lítio e berílio. Não havia elementos mais pesados que esses. Assim, estrelas mais velhas tem metalicidades mais baixas que estrelas mais jovens.

A equação mais utilizada para a metalicidade é $[\mathrm{Fe} / \mathrm{H}]$ :

$$
[F e / H]=\log \left(\frac{N_{F e}}{N_{H}}\right)_{*}-\log \left(\frac{N_{F e}}{N_{H}}\right)_{\odot},
$$

onde $N_{F e}$ e $N_{H}$ correspondem às abundâncias por número de átomos dos elementos Fe e 
$\mathrm{H}$, respectivamente. Os índices $(*)$ e $(\odot)$ referem-se à estrela e ao Sol, respectivamente.

A maior parte das estrelas pobres em metais localiza-se no halo da Galáxia e o estudo de sua cinemática pode indicar se o objeto foi formado nesse ambiente ou se foi acretado ao halo como resultado de processos de interação com outras galáxias e aglomerados. O halo galáctico se estende por alguns milhares de parsecs em escala de altura, e as estrelas descrevem órbitas excêntricas com velocidade orbital média $\mathrm{V} \sim-200 \mathrm{~km} / \mathrm{s}$ (Chiappini 2004) e metalicidade $[\mathrm{Fe} / \mathrm{H}] \sim-2$. De outro lado, tem-se o disco fino com escala de altura entre 220pc e 330pc (Due et al. 2006), objetos de PopI ([Fe/H] 0) descrevendo órbitas mais circulares com velocidade orbital média $\mathrm{V} \sim 220 \mathrm{~km} / \mathrm{s}$ (Chiappini 2004). Já o disco espesso, apresenta escala de altura entre 580pc a 1600pc (Due et al. 2006) e mostra-se como uma estrutura intermediária entre o halo e o disco fino tanto em metalicidade quanto em sua cinemática com velocidade orbital média $\mathrm{V} \sim 150 \mathrm{~km} / \mathrm{s}$ (Chiappini 2004).

$\mathrm{Na}$ astronomia moderna, as estrelas são divididas em classes espectrais que dizem respeito a sua temperatura superficial. Estrelas anãs de classe espectral M são estrelas de sequência principal frias $\left(T_{\text {eff }} \sim 3500 K\right)$ com baixas massas $\left(M \sim 0.5 M_{\odot}\right)$, de modo que seus tempos de vida podem exceder a idade do Universo. Dessa forma, estrelas anãs M pobres em metais (antigas) se mostram com excelentes laboratórios para estudo da evolução química da Galáxia e do Universo.

\subsection{Estrelas de tipo espectral $M$}

Estrelas de tipo espectral M são conhecidas desde os tempos do padre Angelo Secchi. Secchi (1866) classificou-as em 4 tipos com base em suas aparências espectrais (I, II, III, IV). Mais tarde, esses tipos espectrais foram subdivididos e renomeados por Williamina Fleming durante sua classificação de 10351 estrelas para o Draper Memorial Catalogue (Pickering 1890). As estrelas de tipo III classificadas por Secchi tornaram-se as estrelas de tipo M de Fleming. Já naquela época, Secchi notou que os espectros fotográficos dessas estrelas eram dominados por linhas escuras e colunas estriadas, as quais mais tarde foram identificadas por Fowler (1904) como regiões de absorção por moléculas de óxido de titânio (TiO). 
A espectroscopia nos dias de Secchi era limitada aos objetos mais brilhantes, de forma que todas as estrelas de tipo espectral M observadas na época eram gigantes ou super gigantes M (Secchi 1868). A descobertas das anãs M se deu vários anos depois.

\subsubsection{Classificação de temperatura}

Usando as forças das bandas de $\mathrm{TiO}$ como um indicador primário de temperatura (Morgan 1938), foi possível classificar 138 anãs M dentro de um raio de 10pc do Sol (Kuiper 1942), em subclasses espectrais. Na mesma época, Morgan et al. (1943) anunciaram um sistema de classificação para estrelas K e M. No entanto, este último apenas permitia classificar anãs até M2.

Os dois principais sistemas de classificação foram o de Kuiper (1942) e Joy (1947), ambos baseados na força das bandas de TiO. Entretanto, esses sistemas eram discrepantes em até duas subclasses espectrais. Parte das discrepâncias estava no fato de que os espectros de Kuiper estavam na região amarela-vermelha do espectro fotográfico, enquanto os espectros de Joy estavam na região azul. Além disso, Kuiper assumiu que a banda molecular próxima de $6200 \AA$ era unicamente de $\mathrm{TiO}$ enquanto ela é, na verdade, contaminada por hidróxido de cálcio (CaOH). A discrepância foi retificada por Boeshaar (1976) que estabeleceu padrões espectrais de M0 até M6.5 no intervalo de comprimento de onda de 4400-6800 . Em um artigo posterior, Boeshaar \& Tyson (1985) publicaram padrões para M7, M8 e M9 a fim de estender a classificação para os tipos mais frios.

A classificação de toda a sequência de anãs M foi reanalizada para maiores comprimentos de onda (6300-9000^̊) por Kirkpatrick (1991), que estabeleceu padrões espectrais concordantes com os propostos por Boeshaar (1976) e Boeshaar \& Tyson (1985).

\subsubsection{Anãs versus Gigantes e o $\log (g)$}

Nos anos de 1920, tornou-se claro que as estrelas M se dividiam em pelo menos dois segmentos diferentes. Os dados de paralaxe disponíves sugeriam a existência de estrelas M (anãs) que eram de 50 mil a 100 mil vezes menores do que o outro segmento de estrelas M (gigantes) (Lundmark \& Luyten 1923, Luyten 1923b). 
Em vista do limitado número de paralaxes disponíveis na época, começou-se a questior se haveria características espectroscópicas que poderiam separar as duas classes de estrelas. Em 1923, Luyten (1923a) notou que o baixo potencial de ionização de NaI se traduzia em uma nítida diferença na força da linha deste elemento, dada a mudança na densidade do gás entre os dois tipos de objetos. Luyten obteve alguns espectros para testar sua hipótese e mostrou que as linhas de sódio D eram, de fato, mais fortes em anãs do que em gigantes. Burwell (1930) notou a mesma diferença em relação às linhas de cálcio. A força das bandas de hidretos também mostraram-se excelentes discriminantes de luminosidade (Öhman 1934, 1936a, 1936b e Iwanowska \& Wayman 1952).

Atualmente, as estrelas anãs M são definidas como objetos pequenos com raios tipicamente menores do que $0.7 R_{\odot}$ e massas abaixo de $0.6 M_{\odot}$ (López-Morales 2007), enquanto as gigantes $\mathrm{M}$ são objetos com massas $\sim 1-2 M_{\odot}$ e raios variando entre $40 R_{\odot}$ a algumas centenas de $R_{\odot}$ (Wittkowski et al. 2004, 2006b, 2006a). Essas diferenças resultam em $\log (\mathrm{g})$ (unidades do cgs) em torno de 4.5dex para anãs M tipo jovem ("early type") e log(g) em torno de 1.0dex para gigantes M tipo jovem. Essas três ordens e meia de magnitude na gravidade superficial provoca condições físicas muito diferentes na fotosfera desses dois tipos de objetos e, consequentemente, leva às diferenças espectrais observadas.

\subsection{Estrelas subanãs $M$}

Em 1913, Henry Norris Russel reconheceu a grande separação entre as estrelas gigantes e anãs de tipo espectral M. Mais tarde, em 1938, as estrelas anãs foram separadas das subanãs e a primeira subanã M foi catalogada por Gerald P. Kuiper (1938). Inicialmente, Kuiper as classificou como anãs brancas intermediárias. Posteriormente, sugeriu a denominação atual, já que as subanãs eram muito mais brilhantes que as anãs brancas e se assemelhavam mais com as estrelas da sequência principal. Kuiper (1940) também catalogou a primeira estrela subanã M que apresentava luminosidade com duas magnitudes abaixo daquelas de mesmo tipo espectral na sequência principal (anãs M). Hoje, as estrelas subanãs estão localizadas no diagrama HR cobrindo uma faixa de 3 magnitudes abaixo da sequência principal. Seus espectros apresentam anomalias associadas ao baixo conteúdo metálico e a análise cinemática comumente as associam ao disco espesso e halo da Galáxia. A Figura 
(1.1) mostra a localização das subanãs no diagrama HR.

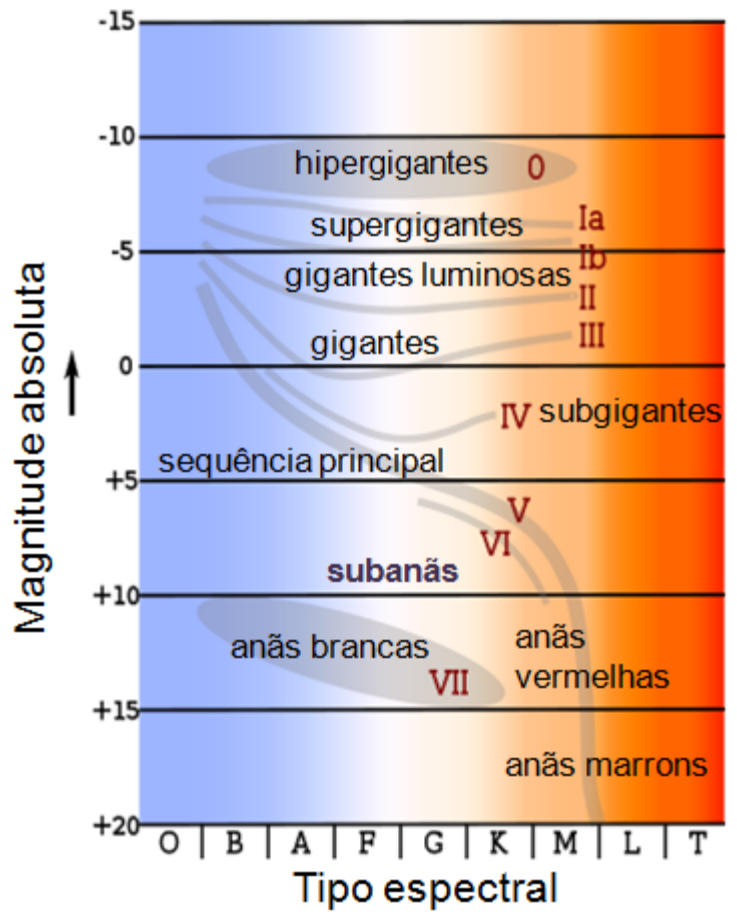

Figura 1.1: Diagrama HR mostrando a localização das estrelas subanãs

\subsubsection{Relação metalicidade/raio}

Quando se compara as subanãs com as anãs de mesma magnitude absoluta, as primeiras apresentam maiores temperaturas superficiais, o que indica que são menores em diâmetro. Além disso, apresentam uma forte emissão na região do ultravioleta, conhecida como excesso do ultravioleta. Essas características são explicadas justamente pela baixa abundância de elementos pesados, os quais, quando presentes nas atmosferas estelares, são responsáveis por aumentar sua opacidade e, consequentemente a pressão de radiação. Na ausência destes, a atmosfera se torna mais transparente, fazendo com que a pressão de radiação não seja tão eficiente para contrabalançar a força gravitacional. Assim, essas estrelas são menores e mais quentes que as anãs ricas em metais.

\subsubsection{Características espectroscópicas}

O espectro no visível e infravermelho das subanãs M (sdM) são dominados por bandas de CO, TiO, AlH, CaH, CrH, FeH, MgH e H2O (Mould \& Hyland 1976; Bessell 1982; Liebert \& Probst 1987; Gizis 1997; Leggett, Allard, \& Hauschildt 1998). Na região do óptico, o 
efeito da metalicidade é visualizado através das bandas dos óxidos e hidretos: à medida que a metalicidade abaixa, as bandas dos hidretos ficam mais fortes e dos óxidos mais fracas (Bidelman \& Smethells 1976; Mould \& Hyland 1976; Bessell 1982), conforme pode ser visto na Figura (1.2). Devido a este efeito, as subanãs vermelhas de alta velocidade, cinematicamente associadas ao halo galáctico (Lépine et al. 2007), consistentemente apresentam bandas de absorção de TiO mais estreitas que as anãs vermelhas mais comuns associadas com o disco galáctico. A razão entre a força das bandas de $\mathrm{TiO}$ e $\mathrm{CaH}$ é conhecida como um diagnóstico de metalicidade (Bessel 1982). No infravermelho próximo, as subanãs são caracterizadas pelo achatamento do espectro (Gray \& Corbally 2009).

\subsubsection{Classificação de metalicidade}

Gizis (1997) foi o primeiro a propor um sistema de classificação de metalicidades para estrelas frias de baixa massa (sub-tipos espectrais (K5-M6) e ultra-frias (M7-M9)). Ele distinguia três grandes classes de metalicidade: as anãs (K5-M9, ou dK5-dM9), as subanãs pobres em metais (sdK5-sdM9) e as subanãs extremamente pobres em metais (esdK5esdM9). A classificação de Gizis (G7) baseia-se em medições espectroscópicas de quatro índices (CaH1, CaH2, CaH3 e TiO5) definidos por Reid et al. (1995). As estrelas anãs $\mathrm{M}(\mathrm{dM})$ exibem bandas fortes de $\mathrm{TiO}$ e $\mathrm{CaH}$, enquanto as subanãs $\mathrm{M}$ pobres em metais (sdM) e subanãs M extremamente pobres (esdM) apresentam bandas relativamente mais fracas de $\mathrm{TiO}$, ao mesmo tempo que exibem absorção forte de $\mathrm{CaH}$ (p. ex. Lépine et al. 2003a; Phan-Bao \& Bessel 2006), comportamento apresentado na Figura(1.2).

Lépine et al. (2007) re-analisaram tal classificação, estudando estrelas com movimentos próprios $\mu>0.45^{\prime \prime}$ ano $^{-1}$, e apontaram uma deficiência na classificação de G7. Através de uma calibração empírica da razão $\mathrm{TiO} / \mathrm{CaH}$ para estrelas na vizinhança solar, eles propuseram uma revisão da classificação, apresentando as sequências de estrelas sdM, esdM e usdM (subanãs ultra pobres em metais) em ordem decrescente de metalicidade. 


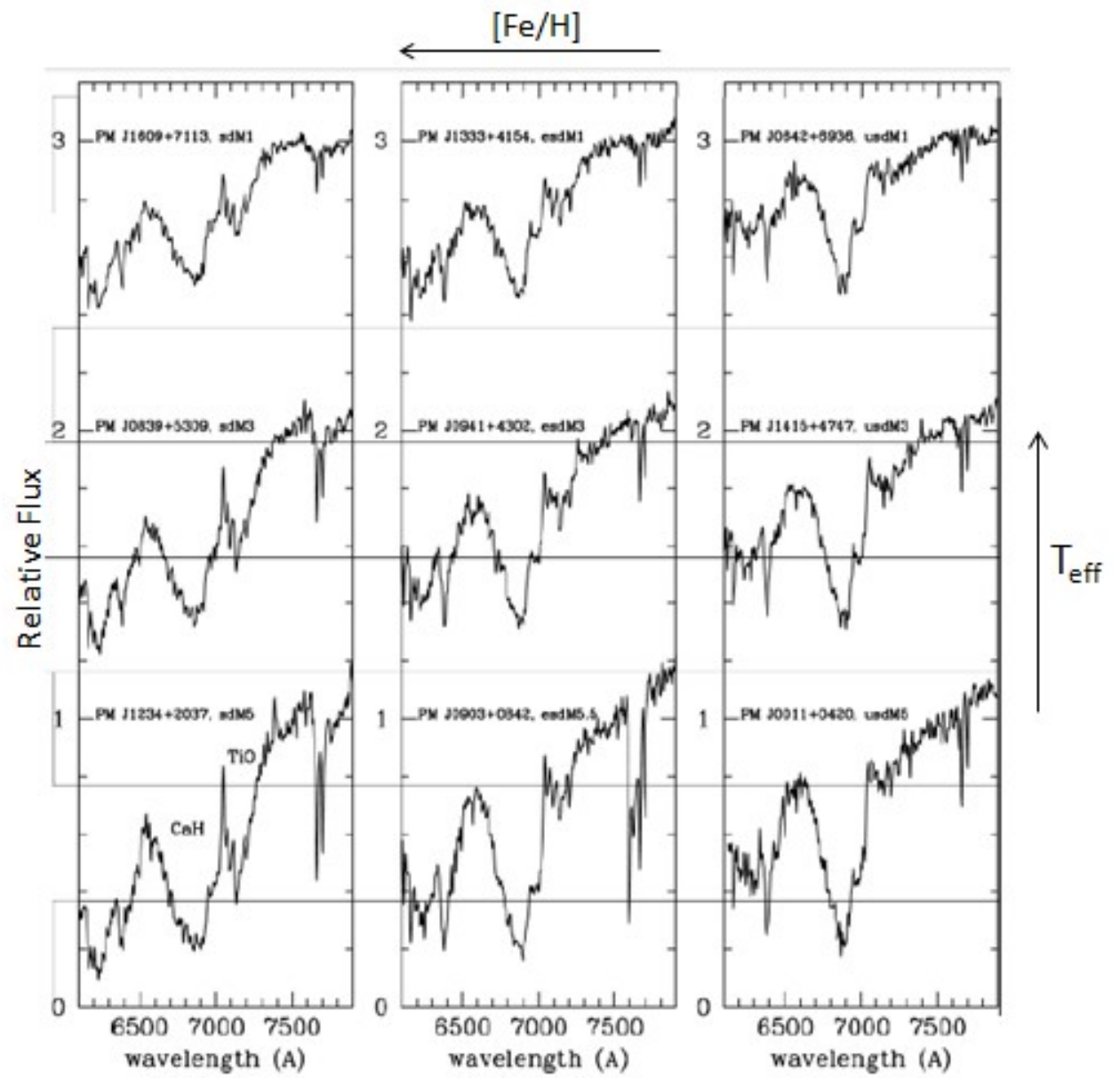

Figura 1.2: Espectros de subanãs frias pobres em metais observadas por S. Lépine no telescópio de 4-m Mayall. Todos os espectros estão normalizados a 1 em $7500 \AA$, e deslocados verticalmente em passos de 1 unidade para clareza. O painel esquerdo mostra a sequência de subanãs M (sdM), o do meio apresenta as subanãs M extremas (esdM) e o painel direito as subanãs ultra pobres em metais (usdM), segundo a classificação de Lépine et al. (2007) (seção 1.3.3). As duas bandas dominantes, fáceis de serem medidas, de $\mathrm{CaH}$ eTiO estão identificadas no espectro esquerdo inferior. As bandas são claramente mais fortes nos objetos mais tardios (mais frios). Note-se também como a razão $\mathrm{TiO} / \mathrm{CaH}$ decresce com a metalicidade. 


\subsubsection{Como separar anãs do disco de subanãs do halo}

As estrelas subanãs de alta velocidade na vizinhança solar, isto é, estrelas de população II, podem ser separadas de estrelas do disco galáctico (população I) usando um diagrama de movimento próprio reduzido, como o sugerido por Jones (1972). Um gráfico do movimento próprio reduzido em função das cores na região entre o óptico e o infravermelho pode fornecer uma clara separação relativa entre anãs $\mathrm{M}$ do disco e subanãs $\mathrm{M}$ do halo galáctico (Salim \& Gould 2002). Com a utilização de tais diagramas, é possível isolar amostras de população I e subanãs de população II e determinar de forma objetiva a separação entre anãs e subanãs $\mathrm{M}$.

Lépine et al. (2007) construiram um diagrama de movimento próprio reduzido utilizando o movimento próprio reduzido na banda V, (equação 1.2) e a cor V-J.

$$
H_{V}=V+5 \log (\mu)+5
$$

Na Figura (1.3), subanãs do halo são separadas das anãs do disco por (1) serem relativamente mais azuis que as anãs para uma dada magnitude absoluta (Monet et al. 1992) e (2) por apresentarem maiores velocidades tranversais. Ambos efeitos se combinam para traçar uma clara separação entre elas.

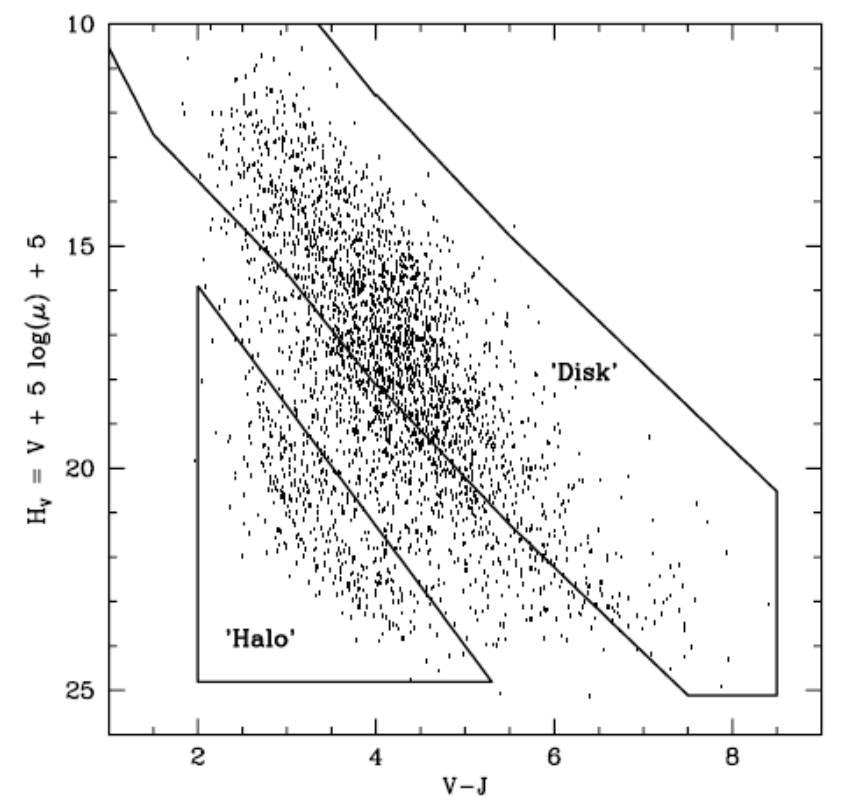

Figura 1.3: Diagrama de movimento próprio reduzido construido por Lépine et al. (2007) para anãs M e subanãs M mostrando a separação entre estrelas do disco e do halo galáctico 


\subsection{Sistemas binários: Detecção}

Sistemas estelares compostos de duas ou mais estrelas (binários e múltiplos) são conhecidos em uma grande variedade de configurações e representam em torno de $50 \%$ das estrelas do Universo. Tais configurações vão de binárias espectroscópicas, fortemente ligadas, com períodos orbitais de dias a anos e separações inferiores a 10 u.a. (Halbwachs et al. 2003) a binárias extensas, fracamente ligadas, com períodos orbitais maiores que $10^{5}$ anos e separações superiores a 20.000 u.a (0,1 pc) (Chanamé \& Gould 2004).

Observações da distribuição de binárias extensas são de particular interesse porque a fraca energia de ligação entre elas faz o sistema ser mais vulnerável a uma ruptura. Modelos teóricos sugerem que sistemas fracamente ligados no disco galáctico irão se desfazer em uma escala de tempo comparável à idade da Galáxia, através de repetidos encontros com outros corpos (Retterer \& King 1982; Weinberg, Shapiro, \& Wasserman 1987; Mallada \& Fernandez 2001).

Existem dois métodos de identificação para detecção destes sistemas na literatura. O primeiro deles visa identificá-las em surveys de imageamento de campo profundo utilizando argumentos baseados na separação angular apenas. A utilização deste método por Bahcall \& Soneira (1981), no entanto, forneceu apenas um número limitado de pares genuínos após confirmação de velocidades radiais por Latham et al. (1984). Surveys adicionais também conduziram a um número restrito de pares.

O segundo método, e de maior sucesso, é baseado na busca por pares CPM (pares de estrelas separadas por alguns segundos ou minutos de arco e que possuem o mesmo movimento próprio) em catálogos de estrelas com grandes movimentos próprios. Nesses catálogos, espera-se encontrar binárias extensas como sendo pares CPM.

Este método de busca foi expandido consideravelmente por W. J. Luyten (1988). Dois catálogos de movimentos próprios foram publicados por Luyten: o LDS (1987) com movimentos próprios pequenos e o LDSS $(1979,1980)$ com altos movimentos próprios $(\mu>$ $\left.0.1^{\prime \prime} / a_{n o}-1\right)$. Um catálogo completo, porém, nunca foi publicado por ele. 
Este trabalho é baseado neste método de seleção de candidatas a pares com altos movimentos próprios. A vantagem na busca por altos movimentos próprios está no fato destas estrelas tenderem a se situar relativamente próximas $(d \leq 100 u . a$.$) , tornando mais fácil a$ identificação de companheiras pouco brilhantes como anãs e subanãs M.

O censo de estrelas com alto movimento próprio $\left(\mu>0.15^{\prime \prime} /\right.$ ano $\left.^{-1}\right)$ aumentou com a publicação do catálogo LSPM-north (95\% completo) por Lépine e Shara (2005). O presente trabalho utiliza candidatas a pares CPM do catálogo complementar LSPM-south (Lépine 2005 e Lépine 2008).

\subsubsection{Subanãs em sistemas binários}

A frequência de anãs M binárias já foi muito estudada. Dentre todas as anãs M conhecidas, de 30\% a 40\% (Jao et al. 2009) delas estão em sistemas binários. Jao et al. (2009) encontraram que 26\% das subanãs $\mathrm{K}$ e M são encontradas em sistemas binários. No entanto, seu estudo ficou incompleto devido à amostra incompleta de subanãs $\mathrm{K}$ e M. Algumas descobertas de subanãs M foram documentadas durante os últimos anos (ex., Gizis 1998; Monet et al. 2000), mas o número total de subanãs primárias ainda permanece pequeno.

\subsection{Este trabalho}

Estrelas anãs e subanãs vermelhas são as estrelas mais abundantes na Gálaxia - representando uma fração > 70\% das estrelas de sequência principal (Bochanski et al. 2010) - e seus tempos de vida são muito maiores que o tempo de Hubble (Laughlin et al. 1997). Com isso, são consideradas extraordinárias traçadoras da evolução química e dinâmica da Galáxia. No entanto, todo este potencial é prejudicado pelas complexas bandas moleculares presentes em seus espectros (seção 1.3.2). Métodos tradicionais de síntese em alta resolução somente são aplicáveis com telescópios de diâmetro da ordem de 10m e para estrelas da vizinhança solar $(d \leq 25 p c$ - PopI). Essa restrição reduz o número de anãs $\mathrm{M}$ pobres em metais do halo galáctico que podem ser estudadas por tais métodos. 
Em vista dessas limitações, técnicas alternativas em baixa/média resolução foram desenvolvidas a fim de estimar as abundâncias químicas dessas estrelas. Em 1995, Reid et al. desenvolveram índices espectroscópicos para medir a força de várias bandas moleculares presentes na região entre $6345 \AA-7120 \AA$, a qual inclui bandas de CaH e TiO. Através desses índices Gizis et al. (1997) classificaram anãs e subanãs M em subclasses de metalicidade (seção 1.3.3). Em 2007, utilizando esses mesmos índices, Lépine et al. desenvolveram um novo índice, que seria um indicador de metalicidade, e reclassificaram as estrelas em novas subclasses de metalicidade.

Observe que, até o momento, falou-se apenas de classes ou subclasses de metalicidades. Um novo desafio para estudo de abundâncias químicas de subanãs M é a calibração de metalicidades, isto é, obter uma equação que correlacione a metalicidade dessas estrelas a um índice extraído do espectro estelar ou de dados fotométricos. Com isso, seria possível estimar a metalicidade de uma subanã M com base apenas em seu espectro em baixa/média resolução ou em sua fotometria. Entretanto, para se obter uma equação de calibração é preciso (1) possuir uma base de dados de estrelas subanãs M e (2) conhecer previamente a metalicidade de todas elas.

É neste ponto que as estrelas subanãs $\mathrm{M}$ em sistemas binários (ou múltiplos) se tornam importantes. Quando se admite que estes sistemas se formaram a partir da mesma nuvem mãe está-se automaticamente afirmando que os membros do sistema compartilham as mesmas abudâncias químicas, de tal forma que se obtém a metalicidade de um deles, temse também a metalicidade do outro membro. A frequência de associações entre estrelas subanãs M com outras estrelas é alta (seção 1.4.1) e um grande número dessas associações com estrelas de tipo espectral F ou G foi catalogado por Lépine et al. (2005 e 2008). Estrelas $\mathrm{F}$ e $\mathrm{G}$ apresentam vários métodos em baixa/média resolução para estimativa de suas metalidades, de forma que essas associações $\mathrm{F} / \mathrm{G}$ - subanã M mostram-se promissoras para a calibração de metalicidades de subanãs $\mathrm{M}$.

O objetivo deste trabalho é, justamente, a recalibração de relações de metalicidades documentadas por Woolf et al. (2009) e Lépine et al. (2012). Enquanto que Woolf et al. (2009) utilizaram uma base de dados pequena em alta resolução que atingia apenas 
$[\mathrm{Fe} / \mathrm{H}] \geq-1.5$ e Lépine et al. (2012) utilizaram apenas pares do hemisfério norte (catálogo LSPM-north), a amostra em estudo é de pares do hemisfério sul.

No Capítulo 2 descreve-se a metodologia utilizada na execução deste trabalho e a seleção da base de dados. No Capítulo 3 apresentam-se as calibrações de metalicidades já documentadas na literatura, ressaltando a escolhida para recalibrar neste trabalho. No Capítulo 4 são expostos e discutidos os resultados para a base de dados e no Capítulo 5 são apresentadas as conclusões deste trabalho. 
Capítulo 2

\section{Metodologia}

\subsection{O catálogo e a seleção}

Os objetos de interesse deste trabalho foram extraídos do catálogo LSPM-south (Lépine, 2005 e Lépine, 2008), atualizado pela última vez em setembro de 2012. Este catálogo é composto por pares extensos de estrelas constituídos de uma estrela primária de tipo espectral F ou G e de outra estrela, secundária, mais fria de tipo espectral M, podendo esta se enquadrar em qualquer classificação de metalicidade descrita no capítulo 1.3.3 (sdM, esdM, usdM) ou mesmo ser uma anã M. Além disso, esses pares apresentam altos movimentos próprios ( $\mu>40$ mas/anos) comuns (CPM - commom proper motion). A detecção destes pares é feita comparando-se suas posições em dois momentos distintos, sendo que o algorítimo está descrito em detalhes em Lépine et al. (2002). Todos os objetos detectados no survey são, então, verificados visualmente e detecções errôneas são excluídas. Para a geração do catálogo foi realizado um survey de estrelas com grandes movimentos próprios, utilizando dados do Digitalized Sky Survey (DSS). As imagens escaneadas do DSS cobrem todo o céu em múltiplas bandas e em várias épocas, sendo o intervalo temporal entre a época mais recente e a mais antiga entre 15 e 45 anos. Os erros estimados para os movimentos próprios do catálogo LSPM-south são de aproximadamente 15 mas/ano.

Com o catálogo em mãos, selecionamos para observação os pares com maior probabilidade de pertencerem ao halo galáctico, já que este é rico em estrelas pobres em metais. Essa seleção foi feita através de um índice cinemático, chamado $K_{\text {index }}$, presente no próprio catálogo e que é diretamente proporcional ao movimento próprio e, segundo os autores, deve estar ligado à metalicidade. A classificação de acordo com este índice é a seguinte: 
- $K_{\text {index }}<0$ 0: Provavelmente indica um sistema muito jovem membro de um aglomerado próximo (Hyades) ou de um grupo de estrelas.

- $0<K_{\text {index }}<1$ : Provavelmente estrelas do disco fino ricas em metal.

- $1<K_{\text {index }}<2$ : Provavelmente estrelas pertencentes ao disco espesso e moderadamente pobres em metal.

- $K_{\text {index }}>2$ : Indica provavelmente estrelas associadas ao halo e pobres em metal.

Com o objetivos de estudar as estrelas com maior probabilidade de serem pobres em metais, selecionamos apenas pares com $K_{\text {index }}>1$. Infelizmente, uma grande parte dos sistemas com $K_{\text {index }}>2$ apresentavam a estrela secundária com magnitude aparente $V \geq 19$, cujos espectros não puderam ser obtidos com razão S/N mínima ( $\geq 20)$ adotada para análise. Assim, nossa amostra é caracterizada por sistemas com $K_{\text {index }}>1$ e $V<19$.

\subsection{Observações e redução dos dados}

Este trabalho utilizou o espectrógrafo Goodman do telescópio SOAR de $4 \mathrm{~m}$ para obtenção dos espectros. O espectrógrafo Goodman fornece espectros com fator de placa típico de $1 \AA /$ pixel. Através de pedidos de tempo, obtivemos 6 noites além de duas doadas pelo grupo colaborador da Michigan State University, totalizando 8 noites de observações. A observação de um par foi feita em duas etapas: (1) observou-se a estrela primária do sistema de tipo F ou G e, imediatamente em seguida, (2) a estrela secundária de tipo M. A seguir descrevem-se as configurações instrumentais para cada uma delas.

\section{Estrela primária:}

As estrelas primárias foram observadas no intervalo de $3500 \AA$ a $6000 \AA$ com uma fenda de 1.35 " de largura e com uma rede de difração de $600 \mathrm{l} / \mathrm{mm}$ para o azul, sem utilização de filtros. O tempo de exposição de cada objeto variou de 3 minutos a 30 minutos de acordo com a magnitude de cada objeto. Após exposição do objeto, uma lâmpada de $\mathrm{Hg}$ e outra de $\mathrm{Cu}$ eram obtidas imediatamente para posterior calibração em comprimento de onda. A razão S/N média para esses objetos ficou acima de 50 com uma média de $\mathrm{S} / \mathrm{N}=150$. 


\section{Estrela secundária:}

As estrelas secundárias foram observadas no intervalo de $6400 \AA$ a $9000 \AA$ com a mesma fenda de $1.35 "$ e com a rede de $600 \mathrm{l} / \mathrm{mm}$ para o vermelho, porém com um filtro vermelho. O tempo de exposição de cada objeto foi em média de 30 minutos, pois todos apresentavam $V>15$. Assim como para a estrela primária, lâmpadas de $\mathrm{Hg}$ e $\mathrm{Cu}$ também foram feitas para calibração. A razão S/N média para esses objetos teve um mínimo de 10 e uma média de $\mathrm{S} / \mathrm{N}=25$.

Em todas as noites de observação o seeing se mostrou menor que 1.0" que era o máximo permitido pelos pedidos de tempo. Todos os objetos também foram observados com massa de ar $<1.2$ a fim de otimizar as observações (mais eficientes e com maior razão $\mathrm{S} / \mathrm{N}$ ).

A redução das estrelas primárias foi feita através de rotinas padrões do IRAF para correção de bias, flatfield e calibração em comprimento de onda. A figura Figura (2.1) apresenta um exemplo de estrela primária da nossa amostra com algumas linhas atômicas indicadas. Para as estrelas secundárias também foram usadas rotinas padrões do IRAF para correção de bias, calibração em comprimento de onda, correção de franjas e calibração em fluxo. Para a correção das franjas utilizamos o procedimento descrito em Goudfrooij et al (1998). A Figura (2.2) mostra a estrela secundarária de PM_I00426-2315 com indicação de algumas bandas moleculares. A Tabela (2.1) mostra os pares observados, suas posições e movimentos próprios e a Tabela (2.2) apresenta as magnitudes 2MASS (Skrutskie et al. 2006). Na Tabela (2.3) encontra-se o número de estrelas observadas em cada noite.

Para efeito de precisão, seguindo a metologia de Lépine et al. (2012), descartamos da análise os pares cuja estrela secundária apresentou $S / N<20$. Com isso, de um total de 37 pares observados, 27 pares apresentaram a secundária com $S / N \geq 20$. Entretanto 5 deles ficaram fora do catálogo LSPM-south com a última atualização feita em setembro de 2012. Portanto, restaram 22 pares para análise neste trabalho. Além dos pares contidos no catálogo, estrelas padrões espectrofotométricas também foram observadas para calibração em fluxo. Essas estrelas estão listadas na Tabela (2.4). 


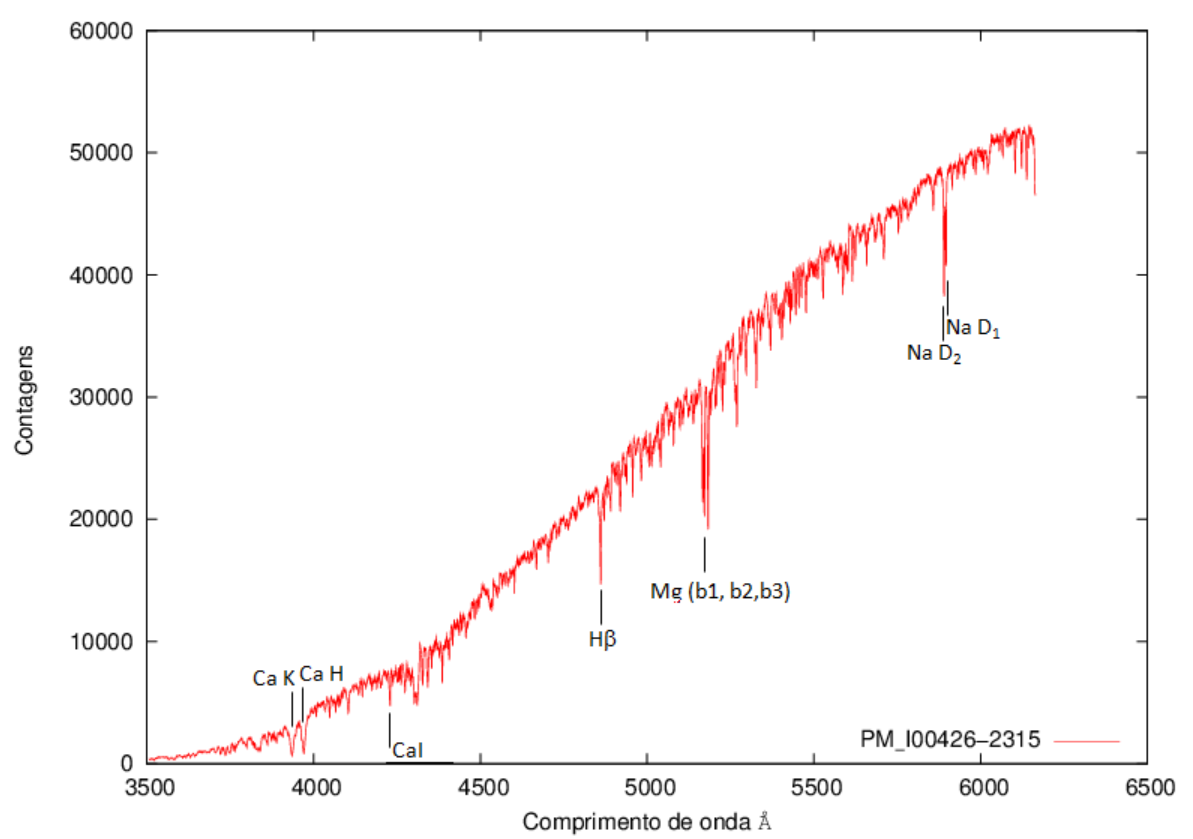

Figura 2.1: Estrela PM_I00426-2315 com indicação de algumas linhas atômicas. Configuração azul. Note que a ordenada está em unidades de contagens.

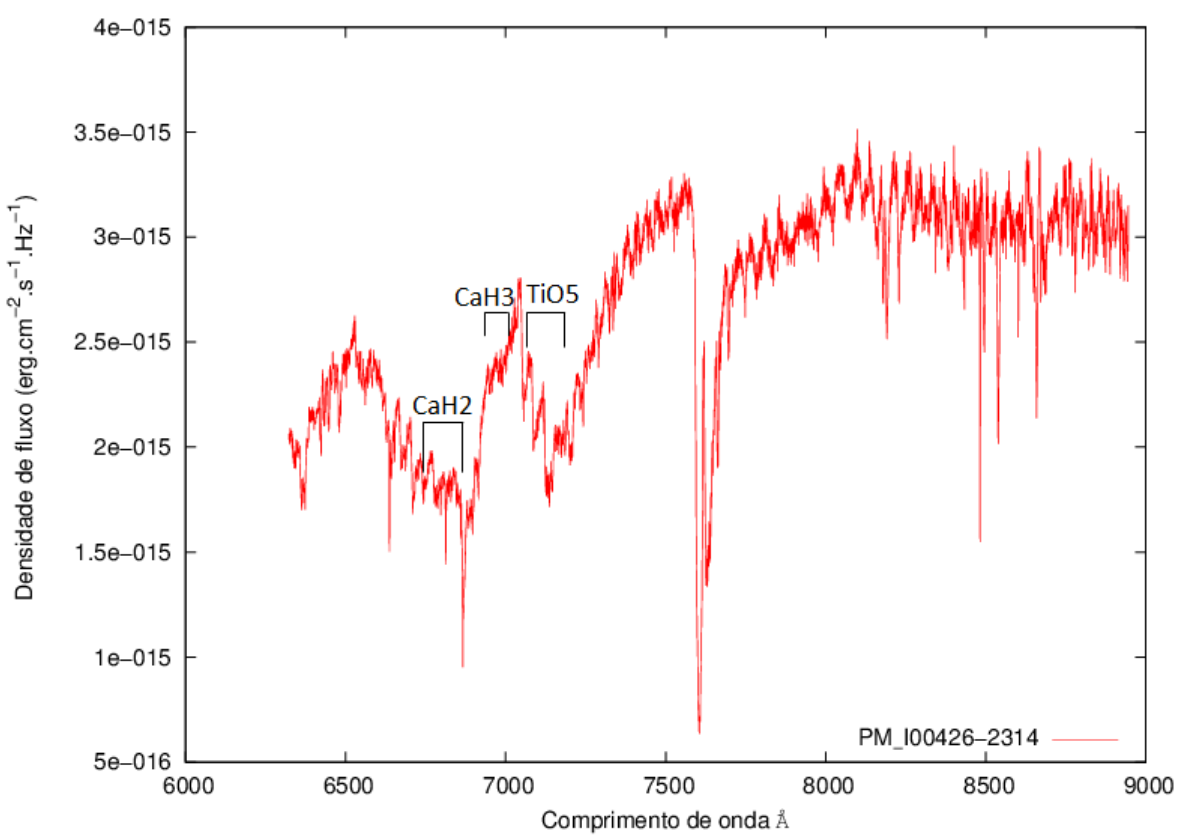

Figura 2.2: Estrela PM_I00426-2314 com indicação de algumas bandas moleculares. Configuração vermelha. Note que a ordenada é apresentada em densidade de fluxo. 
Tabela 2.1 - Posições (J2000) e movimentos próprios para todas as estrelas observadas extraídas do catálogo LSPM-south. A terceira coluna indica se a estrela está presente no catálogo Hipparcos. A coluna 13 indica quais estrelas secundárias apresentam razão $\mathrm{S} / \mathrm{N}>20$ e as duas últimas colunas indicam o $K_{\text {index }}$ e a extinção EBVA para o sistema, respectivamente. Objetos em vermelho indicam sistemas triplos e em azul os pares removidos com a atualização do catálogo.

\begin{tabular}{|c|c|c|c|c|c|c|c|c|c|c|c|c|c|c|}
\hline Par & \multicolumn{6}{|c|}{ Primária } & \multicolumn{6}{|c|}{ Secundária } & \multicolumn{2}{|c|}{ Sistema } \\
\hline Identificação & Nome & HIP & RA(deg) & DEC(deg) & pmRA("/yr) & pmDEC ("/yr) & Nome & RA(deg) & DEC(deg) & pmRA("/yr) & pmDEC ("/yr) & $S / N>20$ & $K_{\text {index }}$ & EBVA \\
\hline 1 & $\mathrm{PM}_{-} \mathrm{I} 00471-2512 \mathrm{~W}$ & - & 11.7755 & -25.20072 & -0.107 & 0.028 & PM_I00471-2514N & 11.7922 & -25.2353 & -0.096 & 0.034 & não & 1.8 & 0.019 \\
\hline 2 & PM_I01008-2125 & - & 15.2006 & -21.43292 & 0.117 & 0.028 & PM_I01008-2126 & 15.2036 & -21.4345 & 0.117 & 0.028 & $\operatorname{sim}$ & 1.1 & 0.019 \\
\hline 3 & $\mathrm{PM}_{-} \mathrm{I} 00426-2315$ & - & 10.6570 & -23.2508 & 0.122 & -0.027 & PM_I00426-2314 & 10.6604 & -23.2429 & 0.132 & -0.024 & $\operatorname{sim}$ & 1.9 & 0.018 \\
\hline 4 & PM_I01266-4842W & - & 21.6555 & -48.71417 & 0.205 & -0.05 & $\mathrm{PM}_{-} \mathrm{I} 01266-4842 \mathrm{E}$ & 21.6595 & -48.7153 & 0.205 & -0.05 & $\operatorname{sim}$ & 1.4 & 0.010 \\
\hline 5 & $\mathrm{PM}_{-} 101543-2249 \mathrm{~S}$ & - & 28.5784 & -22.826 & -0.019 & -0.171 & $\mathrm{PM}_{-} \mathrm{I01543-2249 \textrm {N }}$ & 28.5756 & -22.8232 & -0.008 & -0.167 & $\operatorname{sim}$ & 1.6 & 0.010 \\
\hline 6 & $\mathrm{PM}_{-} \mathrm{I} 02300-2402 \mathrm{~W}$ & - & 37.5022 & -24.03886 & -0.144 & -0.148 & PM_I02299-2401Ss & 37.4935 & -24.0324 & -0.156 & -0.157 & não & 1.8 & 0.019 \\
\hline 6 & $\mathrm{PM}_{-} \mathrm{I} 02300-2402 \mathrm{~W}$ & - & 37.5022 & -24.03886 & -0.144 & -0.148 & $\mathrm{PM}_{-} \mathrm{I} 02300-2402 \mathrm{E}$ & 37.5195 & -24.0456 & -0.171 & -0.153 & $\operatorname{sim}$ & 2.5 & 0.019 \\
\hline 7 & $\mathrm{PM}_{-} \mathrm{I} 02463-2312$ & - & 41.5751 & -23.2074 & -0.058 & -0.111 & PM_I02462-2311 & 41.5707 & -23.1932 & -0.066 & -0.105 & $\operatorname{sim}$ & 2.2 & 0.029 \\
\hline 8 & $\mathrm{PM}_{-} \mathrm{I} 02542-6620$ & - & 43.5694 & -66.34064 & 0.114 & 0.013 & $\mathrm{PM}_{-} \mathrm{I} 02542-6619$ & 43.5712 & -66.3215 & 0.12 & 0.016 & $\operatorname{sim}$ & 1.1 & 0.029 \\
\hline 9 & PM_I04325-5657N & - & 68.1352 & -56.95119 & 0.124 & -0.133 & $\mathrm{PM}_{-} \mathrm{I} 04325-5657 \mathrm{~S}$ & 68.1325 & -56.9541 & 0.124 & -0.133 & $\operatorname{sim}$ & 1.3 & 0.010 \\
\hline 10 & $\mathrm{PM}_{-} \mathrm{I} 04477-3044 \mathrm{~W}$ & - & 71.9277 & -30.73422 & 0.157 & -0.142 & PM_I04477-3044E & 71.9342 & -30.7341 & 0.143 & -0.143 & $\operatorname{sim}$ & 2.2 & 0.023 \\
\hline 11 & PM_I05418-5000 & 26830 & 85.4554 & -50.00436 & 0.062 & 0.079 & PM_I05418-5002 & 85.4650 & -50.0345 & 0.065 & 0.062 & não & 1.5 & 0.032 \\
\hline 12 & PM_I $05425-4305 E$ & - & 85.6496 & -43.09861 & -0.025 & -0.118 & PM_I05425-4305W & 85.6449 & -43.1000 & -0.025 & -0.118 & $\operatorname{sim}$ & 1.3 & 0.055 \\
\hline 13 & PM_I05442-2416 & - & 86.0640 & -24.27628 & -0.086 & 0.062 & $\mathrm{PM}_{-} \mathrm{I} 05442-2417 \mathrm{~N}$ & 86.0651 & -24.2839 & -0.093 & 0.064 & $\operatorname{sim}$ & 1 & 0.029 \\
\hline 14 & $\mathrm{PM}_{-} \mathrm{I0559-4511}$ & 28062 & 88.9984 & -45.18956 & 0.093 & 0.208 & PM_I05560-4511 & 89.0073 & -45.1898 & 0.098 & 0.189 & $\operatorname{sim}$ & 1.2 & 0.065 \\
\hline 15 & PM_I06276-2355W & - & 96.9116 & -23.929 & -0.011 & 0.099 & PM_I06276-2356 & 96.9008 & -23.9442 & -0.008 & 0.099 & $\operatorname{sim}$ & 1.2 & 0.061 \\
\hline 16 & $\mathrm{PM}_{-} \mathrm{I} 08239-7549 \mathrm{~W}$ & - & 125.9790 & -75.82625 & -0.045 & 0.202 & PM_I08239-7549E & 125.9939 & -75.8257 & -0.03 & 0.216 & $\operatorname{sim}$ & 1.6 & 0.155 \\
\hline 17 & PM_I08386-3856 & - & 129.6530 & -38.94881 & 0.101 & -0.126 & PM_I08386-3857 & 129.6572 & -38.9540 & 0.101 & -0.126 & $\operatorname{sim}$ & 1.5 & 1.087 \\
\hline 18 & PM_I13133-4153N & - & 198.3354 & -41.88722 & -0.145 & -0.044 & PM_I13133-4153S & 198.3381 & -41.8914 & -0.145 & -0.044 & $\operatorname{sim}$ & 1.8 & 0.103 \\
\hline 19 & PM_I18240-5107N & - & 276.0035 & -51.12936 & 0.039 & -0.154 & PM_I18241-5107 & 276.0292 & -51.1180 & 0.036 & -0.154 & $\operatorname{sim}$ & 1 & 0.077 \\
\hline 19 & PM_I18240-5107S & 90171 & 276.0030 & -51.13036 & 0.039 & -0.154 & PM_I18241-5107 & 276.0292 & -51.1180 & 0.036 & -0.154 & $\operatorname{sim}$ & 1 & 0.077 \\
\hline 20 & PM_I20345-2219 & - & 308.6312 & -22.32344 & 0.143 & -0.108 & PM_I20345-2217 & 308.6395 & -22.3000 & 0.133 & -0.099 & não & 1.5 & 0.048 \\
\hline 21 & PM_I20371-5815 & - & 309.2913 & -58.26625 & 0.134 & -0.105 & PM_I $20371-5816$ & 309.2952 & -58.2681 & 0.134 & -0.105 & $\operatorname{sim}$ & 1 & 0.074 \\
\hline 22 & PM_I21175-4142E & - & 319.3845 & -41.7048 & 0.013 & -0.157 & PM_I21175-4142W & 319.3809 & -41.7059 & 0.013 & -0.157 & $\operatorname{sim}$ & 2.3 & 0.031 \\
\hline 23 & PM_I21229-7141 & - & 320.7376 & -71.69842 & 0.055 & -0.1 & PM_I $121229-7142$ & 320.7267 & -71.7154 & 0.055 & -0.1 & não & 2.4 & 0.048 \\
\hline 24 & PM_I21545-7102 & - & 328.6482 & -71.03567 & 0.096 & -0.011 & PM_I $21547-7103$ & 328.6838 & -71.0598 & 0.096 & -0.011 & não & 1.3 & 0.026 \\
\hline 25 & $\mathrm{PM}_{-} \mathrm{I} 22487-5613 \mathrm{~W}$ & - & 342.1847 & -56.22694 & 0.145 & -0.067 & $\mathrm{PM}_{-} \mathrm{I} 22487-5613 \mathrm{E}$ & 342.1897 & -56.2289 & 0.145 & -0.067 & $\operatorname{sim}$ & 1.3 & 0.013 \\
\hline 26 & PM_I23033-5311 & - & 345.8478 & -53.18975 & 0.148 & -0.107 & PM_I23034-5311 & 345.8575 & -53.1954 & 0.153 & -0.098 & $\operatorname{sim}$ & 2 & 0.006 \\
\hline 27 & PM_I23099-2057 & - & 347.4956 & -20.9564 & 0.212 & -0.14 & PM_I23099-2056 & 347.4998 & -20.9438 & 0.186 & -0.136 & $\operatorname{sim}$ & 2.6 & 0.031 \\
\hline 28 & PM_I $23324-2154 \mathrm{~W}$ & - & 353.1128 & -21.91492 & 0.115 & -0.251 & PM_I $23324-2154 \mathrm{E}$ & 353.1158 & -21.9142 & 0.13 & -0.257 & não & 1.6 & 0.029 \\
\hline 29 & $\mathrm{PM}_{-} \mathrm{I00251-2354 \textrm {E }}$ & - & 6.2928 & -23.90364 & 0.065 & -0.129 & $\mathrm{PM}_{-} \mathrm{I} 00251-2354 \mathrm{~W}$ & 6.2829 & -23.9124 & 0.065 & -0.129 & $\operatorname{sim}$ & 1.8 & 0.016 \\
\hline 30 & $\mathrm{PM}_{-} \mathrm{I} 07365-4751 \mathrm{Sn}$ & - & 114.1275 & -47.86211 & 0.159 & -0.169 & PM_I07365-4751Ss & 114.1256 & -47.8643 & 0.159 & -0.169 & não & 1.4 & 0.203 \\
\hline 31 & PM_I08152-6337 & - & 123.8233 & -63.62178 & -0.152 & 0.24 & PM_I08153-6337 & 123.8251 & 63.6179 & -0.152 & 0.24 & $\operatorname{sim}$ & 1.8 & 0.174 \\
\hline 32 & PM_I14568-2704N & - & 224.2059 & -27.06925 & -0.174 & 0.005 & PM_I14568-2704S & 224.2041 & -27.0712 & -0.174 & 0.005 & não & 1.4 & 0.145 \\
\hline 33 & PM_I19145-7109 & - & 288.6420 & -71.16331 & -0.003 & -0.223 & PM_I19146-7109N & 288.6650 & -71.1584 & -0.036 & -0.22 & $\operatorname{sim}$ & 1.4 & 0.058 \\
\hline 34 & PM_I20295-5757 & 101091 & 307.3856 & -57.95775 & 0.287 & -0.091 & PM_I20297-5757 & 307.4428 & -57.9647 & 0.318 & -0.119 & $\operatorname{sim}$ & 1.2 & 0.071 \\
\hline 35 & PM_I22287-4915N & 110952 & 337.1947 & -49.26539 & 0.19 & -0.092 & PM_I $22288-4914$ & 337.2195 & -49.2366 & 0.165 & -0.071 & não & 1.3 & 0.013 \\
\hline $36^{*}$ & PM_I01147-4536 & - & 18.6775 & -45.6096 & -0.109 & -0.126 & não observada & - & - & - & - & - & - & - \\
\hline $37^{*}$ & PM_I03317-2119 & - & 52.9458 & -21.32275 & 0.077 & -0.04 & não observada & - & - & - & - & - & - & - \\
\hline $38^{*}$ & PM_I03533-2313 & - & 58.3283 & -23.2209 & -0.122 & -0.018 & não observada & - & - & - & - & - & - & - \\
\hline $39 *$ & $\mathrm{PM}_{-} \mathrm{I} 06291-2305 \mathrm{Wn}$ & - & 97.2851 & -23.08714 & -0.102 & 0.021 & não observada & - & - & - & - & - & - & - \\
\hline $40^{*}$ & PM_I07194-7422 & - & 109.8504 & -74.37881 & 0.012 & -0.123 & não observada & - & - & - & - & - & . & . \\
\hline
\end{tabular}

* A companheira vermelha não foi observada ou por erro de identificação no céu ou em vista do amanhecer. 
Tabela 2.2 - Fotometria para todas as estrelas observadas extraídas do catálogo LSPM-south. A terceira coluna indica se a estrela está presente no catálogo Hipparcos. A coluna 13 indica quais estrelas secundárias apresentam razão $\mathrm{S} / \mathrm{N}>20$ e as duas últimas colunas indicam o $K_{\text {index }}$ e a extinção EBVA para o sistema, respectivamente. Objetos em vermelho indicam sistemas triplos e em azul os pares removidos com a atualização do catálogo.

\begin{tabular}{|c|c|c|c|c|c|c|c|c|c|c|c|c|c|c|}
\hline \multirow{2}{*}{$\begin{array}{c}\text { Par } \\
\text { Identificação }\end{array}$} & \multicolumn{6}{|c|}{ Primária } & \multicolumn{6}{|c|}{ Secundária } & \multicolumn{2}{|c|}{ Sistema } \\
\hline & Nome & HIP & $\mathrm{V}$ & $\mathrm{J}$ & $\mathrm{H}$ & $\mathrm{K}$ & Nome & $\mathrm{V}$ & $\mathrm{J}$ & $\mathrm{H}$ & $\mathrm{K}$ & $S / N>20$ & $K_{\text {index }}$ & EBVA \\
\hline 1 & $\mathrm{PM}_{-} \mathrm{I} 00471-2512 \mathrm{~W}$ & - & 13.33 & 11.8 & 11.48 & 11.44 & $\mathrm{PM}_{-} \mathrm{I} 00471-2514 \mathrm{~N}$ & 18.06 & 15.05 & 14.38 & 14.19 & não & 1.8 & 0.019 \\
\hline 2 & PM_I01008-2125 & - & 12.9 & 11.36 & 10.92 & 10.83 & PM_I01008-2126 & 16.46 & 13.17 & 12.65 & 12.45 & $\operatorname{sim}$ & 1.1 & 0.019 \\
\hline 3 & PM_I00426-2315 & - & 13.17 & 11.73 & 11.4 & 11.33 & $\mathrm{PM}_{-} \mathrm{I} 00426-2314$ & 17.36 & 14.43 & 13.87 & 13.61 & $\operatorname{sim}$ & 1.9 & 0.018 \\
\hline 4 & PM_I01266-4842W & - & 13.3 & 11.54 & 10.86 & 10.73 & $\mathrm{PM}_{-} \mathrm{I} 01266-4842 \mathrm{E}$ & 18.76 & 14.43 & 13.94 & 13.7 & $\operatorname{sim}$ & 1.4 & 0.010 \\
\hline 5 & $\mathrm{PM}_{-} \mathrm{I} 01543-2249 \mathrm{~S}$ & - & 11.22 & 9.91 & 9.5 & 9.44 & $\mathrm{PM}_{-} \mathrm{I} 01543-2249 \mathrm{~N}$ & 16.96 & 13.68 & 13.11 & 12.93 & $\operatorname{sim}$ & 1.6 & 0.010 \\
\hline 6 & $\mathrm{PM}_{-} \mathrm{I} 02300-2402 \mathrm{~W}$ & - & 13.86 & 10.65 & 12.08 & 11.98 & $\mathrm{PM} \_\mathrm{I} 02299-2401 \mathrm{Ss}$ & 18.64 & 14.66 & 14.02 & 13.71 & não & 1.8 & 0.019 \\
\hline 6 & $\mathrm{PM}_{-} \mathrm{I} 02300-2402 \mathrm{~W}$ & - & 13.86 & 12.39 & 12.08 & 11.98 & $\mathrm{PM}_{-} \mathrm{I} 02300-2402 \mathrm{E}$ & $\mid 18.7$ & 15.36 & 14.88 & 14.44 & $\operatorname{sim}$ & 2.5 & 0.019 \\
\hline 7 & PM_I02463-2312 & - & 13.45 & 12.09 & 11.72 & 11.65 & PM_I02462-2311 & 18.43 & 15.47 & 14.74 & 14.63 & $\operatorname{sim}$ & 2.2 & 0.029 \\
\hline 8 & $\mathrm{PM}_{-} \mathrm{I} 02542-6620$ & - & 9.85 & 8.59 & 8.32 & 8.21 & $\mathrm{PM}_{-} \mathrm{I} 02542-6619$ & 17.4 & 13.74 & 13.13 & 13.01 & $\operatorname{sim}$ & 1.1 & 0.029 \\
\hline 9 & $\mathrm{PM}_{-} \mathrm{I} 04325-5657 \mathrm{~N}$ & - & 12.16 & 10.88 & 10.51 & 10.45 & PM_I04325-5657S & 17.38 & 13.65 & 13.18 & 12.89 & $\operatorname{sim}$ & 1.3 & 0.010 \\
\hline 10 & PM_I04477-3044W & - & 11.51 & 10.28 & 9.92 & 9.90 & PM_I $04477-3044 \mathrm{E}$ & 18.48 & 15.04 & 14.53 & 14.35 & $\operatorname{sim}$ & 2.2 & 0.023 \\
\hline 11 & PM_I05418-5000 & 26830 & 8.82 & 7.2 & 6.82 & 6.74 & PM_I05418-5002 & 18.87 & 15.28 & 14.8 & 14.6 & não & 1.5 & 0.032 \\
\hline 12 & $\mathrm{PM}_{-} 105425-4305 \mathrm{E}$ & - & 12.16 & 10.72 & 10.23 & 10.13 & $\mathrm{PM}_{-} 105425-4305 \mathrm{~W}$ & 18.05 & 14.33 & 13.78 & 13.56 & $\operatorname{sim}$ & 1.3 & 0.055 \\
\hline 13 & PM_I05442-2416 & - & 11.65 & 10.01 & 9.51 & 9.44 & $\mathrm{PM}_{-} \mathrm{I} 05442-2417 \mathrm{~N}$ & 17.02 & 13.47 & 12.88 & 12.65 & $\operatorname{sim}$ & 1 & 0.029 \\
\hline 14 & PM_I0559-4511 & 28062 & 10.51 & 8.9 & 8.51 & 8.40 & PM_I05560-4511 & 17.29 & 13.28 & 12.7 & 12.42 & $\operatorname{sim}$ & 1.2 & 0.065 \\
\hline 15 & PM_I06276-2355W & - & 13.29 & 11.91 & 11.61 & 11.56 & PM_I06276-2356 & 17.53 & 14.15 & 13.57 & 13.36 & $\operatorname{sim}$ & 1.2 & 0.061 \\
\hline 16 & PM_I08239-7549W & - & 10.96 & 9.53 & 9.08 & 8.99 & $\mathrm{PM}_{-} \mathrm{108239-7549 \textrm {E }}$ & 16.5 & 13.23 & 12.68 & 12.39 & $\operatorname{sim}$ & 1.6 & 0.155 \\
\hline 17 & PM_I08386-3856 & - & 12.59 & 10.73 & 10.21 & 10.15 & $\mathrm{PM}_{-} \mathrm{I} 08386-3857$ & 17.36 & 13.85 & 13.35 & 13.16 & $\operatorname{sim}$ & 1.5 & 1.087 \\
\hline 18 & PM_I13133-4153N & - & 13.58 & 11.58 & 10.95 & 10.89 & $\mathrm{PM}_{-} \mathrm{I} 13133-4153 \mathrm{~S}$ & 18.15 & 14.7 & 14.2 & 13.98 & $\operatorname{sim}$ & 1.8 & 0.103 \\
\hline 19 & $\mathrm{PM}_{-} \mathrm{I} 18240-5107 \mathrm{~N}$ & - & 11.99 & 9.96 & 8.7 & 9.38 & PM_I18241-5107 & 18.13 & 13.87 & 13.31 & 13.06 & $\operatorname{sim}$ & 1 & 0.077 \\
\hline 19 & PM_I18240-5107S & 90171 & 10.28 & 9.32 & 8.62 & 8.89 & PM_I18241-5107 & 18.13 & 13.87 & 13.31 & 13.06 & $\operatorname{sim}$ & 1 & 0.077 \\
\hline 20 & PM_I20345-2219 & - & 11.07 & 9.88 & 9.54 & 9.52 & PM_I $20345-2217$ & 18.92 & 14.79 & 14.22 & 14.03 & não & 1.5 & 0.048 \\
\hline 21 & PM_I20371-5815 & - & 13.3 & 11.26 & 10.64 & 10.47 & PM_I20371-5816 & 16.84 & 13.05 & 12.44 & 12.2 & $\operatorname{sim}$ & 1 & 0.074 \\
\hline 22 & PM_I $121175-4142 \mathrm{E}$ & - & 13.83 & 12.15 & 11.78 & 11.68 & PM_I $121175-4142 \mathrm{~W}$ & 17.57 & 14.8 & 14.22 & 13.95 & $\operatorname{sim}$ & 2.3 & 0.031 \\
\hline 23 & PM_I21229-7141 & - & 15.21 & 13.77 & 13.43 & 13.37 & PM_I21229-7142 & 19.53 & 16.39 & 15.65 & 15.57 & não & 2.4 & 0.048 \\
\hline 24 & PM_I21545-7102 & - & 15.41 & 13.23 & 12.66 & 12.48 & PM_I21547-7103 & 18.23 & 14.69 & 13.91 & 13.77 & não & 1.3 & 0.026 \\
\hline 25 & $\mathrm{PM}_{-} \mathrm{I} 22487-5613 \mathrm{~W}$ & - & 12.51 & 10.99 & 10.51 & 10.42 & $\mathrm{PM}_{-} \mathrm{I} 22487-5613 \mathrm{E}$ & 16.66 & 13.26 & 12.69 & 12.44 & $\operatorname{sim}$ & 1.3 & 0.013 \\
\hline 26 & PM_I23033-5311 & - & 13.35 & 12.25 & 11.91 & 11.85 & PM_I23034-5311 & 19 & 15.31 & 14.81 & 14.5 & $\operatorname{sim}$ & 2 & 0.006 \\
\hline 27 & PM_I23099-2057 & - & 12.32 & 10.69 & 10.4 & 10.39 & PM_I23099-2056 & 17.49 & 14.75 & 14.2 & 13.96 & $\operatorname{sim}$ & 2.6 & 0.031 \\
\hline 28 & PM_I $23324-2154 \mathrm{~W}$ & - & 10.93 & 9.63 & 9.29 & 9.20 & PM_I $23324-2154 \mathrm{E}$ & 20.23 & 15.23 & 14.83 & 14.5 & não & 1.6 & 0.029 \\
\hline 29 & $\mathrm{PM}_{-} \mathrm{I} 00251-2354 \mathrm{E}$ & - & 14.55 & 12.47 & 11.99 & 11.86 & $\mathrm{PM}_{-} \mathrm{100251-2354 \textrm {W }}$ & 18.13 & 14.74 & 14.22 & 14.091 & $\operatorname{sim}$ & 1.8 & 0.016 \\
\hline 30 & PM_I07365-4751Sn & - & 11.96 & 9.66 & 9.08 & 8.92 & PM_I07365-4751Ss & 19 & 14.43 & 13.86 & 13.35 & não & 1.4 & 0.203 \\
\hline 31 & $\mathrm{PM}_{-} \mathrm{I} 08152-6337$ & - & 12.16 & 9.96 & 9.37 & 9.25 & PM_I08153-6337 & 16.66 & 13.28 & 12.67 & 12.402 & $\operatorname{sim}$ & 1.8 & 0.174 \\
\hline 32 & PM_I14568-2704N & - & 12.53 & 10.23 & 9.61 & 9.47 & PM_I14568-2704S & 17.77 & 14.06 & 13.46 & 13.189 & não & 1.4 & 0.145 \\
\hline 33 & PM_I19145-7109 & - & 10.18 & 8.69 & 8.34 & 8.25 & PM_I19146-7109N & 17.9 & 13.81 & 13.24 & 12.937 & $\operatorname{sim}$ & 1.4 & 0.058 \\
\hline 34 & PM_I20295-5757 & 101091 & 9.39 & 8.07 & 7.73 & 7.59 & PM_I20297-5757 & 16.4 & 12.38 & 11.79 & 11.557 & $\operatorname{sim}$ & 1.2 & 0.071 \\
\hline 35 & PM_I $122287-4915 \mathrm{~N}$ & 110952 & 10.59 & 8.83 & 8.31 & 8.22 & PM_I22288-4914 & 19.79 & 15.11 & 14.57 & 14.3 & não & 1.3 & 0.013 \\
\hline $36^{*}$ & PM_I01147-4536 & - & 14.04 & 12.43 & 12.1 & 12.07 & não observada & - & - & - & - & - & - & - \\
\hline $37^{*}$ & PM_I03317-2119 & - & 10.41 & 9.25 & 8.97 & 8.93 & não observada & - & - & - & - & - & - & 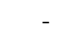 \\
\hline $38^{*}$ & PM_I03533-2313 & - & 14.36 & 13.05 & 12.68 & 12.62 & não observada & - & - & - & - & - & - & - \\
\hline $39 *$ & PM_I06291-2305Wn & - & 15.19 & 13.11 & 12.8 & 12.71 & não observada & - & - & - & - & - & 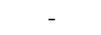 & - \\
\hline $40^{*}$ & PM_I07194-7422 & - & 11.84 & 10.47 & 9.98 & 9.87 & não observada & . & - & - & - & - & - & - \\
\hline
\end{tabular}

* A companheira vermelha não foi observada ou por erro de identificação no céu ou em vista do amanhecer. 
Tabela 2.3 - Número de estrelas observadas em cada noite ao longo de 3 semestres.

\begin{tabular}{|c|c|c|c|c|}
\hline & \multicolumn{2}{|c|}{$2011 \mathrm{~B}$} & $2012 \mathrm{~A}$ & $2012 \mathrm{~B}$ \\
\hline \hline Mês & Outubro & Dezembro & Julho & Outubro \\
\hline Número de noites & 2 (doadas pela MSU) & 3 & 2 & 1 \\
\hline \hline Estrelas observadas & 19 & 34 & 19 & 10 \\
\hline Total de estrelas & \multicolumn{3}{|c|}{82} \\
\hline \hline Pares completos & 9 & 16 & $8+1$ sistema triplo & 4 \\
\hline Pares utilizados na análise & 2 & 12 & $3+1$ sistema triplo & 4 \\
\hline Total de pares para análise & \multicolumn{3}{|c|}{$21+1$ sistema triplo } \\
\hline
\end{tabular}

Tabela 2.4 - Estrelas padrões espectrofotométricas observadas para calibração em fluxo.

\begin{tabular}{|c|c|c|c|}
\hline \multicolumn{2}{|c|}{ 2011B } & 2012A & 2012B \\
\hline \hline Outubro & Dezembro & Julho & Outubro \\
\hline- & HD49798 & $\begin{array}{c}\text { LTT377 } \\
\text { LTT7377 }\end{array}$ & $\begin{array}{c}\text { LTT2415 } \\
\text { - }\end{array}$ \\
\hline
\end{tabular}

\subsection{Como os dados foram obtidos}

Como descrito anteriormente, os pares estelares presentes no catálogo LSPM-south são formados por uma estrela de tipo F ou G e outra de tipo M. Para obtenção e interpretação dos resultados considerou-se que esses pares foram gerados a partir da mesma nuvem mãe, de tal forma que compartilham a mesma metalicidade. Assim, obtendo-se a metalicidade de uma delas, automaticamente determina-se a metalicidade da companheira. É com essa suposição que se estima a metalicidades para as subanãs $\mathrm{M}$ observadas neste trabalho. Mesmo não tendo certeza se o par se originou de uma mesma nuvem, pode-se confirmar espectroscopicamente se o sistema realmente forma um par físico. Como esses sistemas são extensos, os membros devem compartilhar dentro de certa precisão a mesma velocidade radial e assim pode-se confirmar se o sistema realmente constitui um par estelar. 
Para estimativa de metalicidade da estrela primária utilizou-se o n-sspp, uma revisão modificada do SEGUE Stellar Parameter Pipeline (SSPP, ver Lee et al. 2008a, Lee et al. 2008b, Prieto et al. 2008, Smolinski et al. 2011, Lee et al. 2011 para uma descrição detalhada dos procedimentos empregados). O n-sspp é uma ferramenta bem desenvolvida e testada para estimativa de parâmetros atmosféricos de estrelas com espectros disponíveis em baixa e média resolução. Foi especificamente desenhado para processar grandes quantidades de dados de forma automática, tornando-o ideal para os dados deste projeto. O n-sspp também fornece velocidades radiais através do efeito Doppler, além de estimar distâncias. Se o par é físico, a estimativa da metalicidade da estrela primária via n-sspp, fornece também a metalicidade da secundária M.

A calibração de metalicidades das estrelas subanãs é feita via correlação entre a metalicidade obtida para a estrela primária com índices de linhas extraídos dos espectros dessas estrelas. Neste trabalho, tal correlação foi feita com um índice descrito em Lépine et al. (2007), chamado $\zeta_{\mathrm{TiO} / \mathrm{CaH}}$, que se baseia nas forças das bandas de TiO5, CaH2 e CaH3. Ao correlacionar a metalicidade $\operatorname{com} \zeta_{\mathrm{TiO} / \mathrm{CaH}}$ pode-se verificar quão bem este índice funciona como um indicador de metalicidade. 
Capítulo 3

\section{Calibrações de metalicidades}

Neste capítulo apresenta-se as calibrações tanto fotométricas quanto espectroscópicas para calibração de metalicidades de estrelas subanãs M existentes na literatura, bem como, adicionais sobre a relação escolhida.

\subsection{Calibrações fotométricas}

Na literatura, existem muitas propostas de calibrações fotométricas para subanãs M. A seguir, apresenta-se algumas delas.

(a) Bonfils et al. (2005):

A primeira calibração realizada pelos autores utilizou a correlação entre a posição de uma estrela anã M num diagrama cor-magnitude $V-K_{S}-M_{K_{S}}$ e metalicidade. A relação obtida foi:

$$
[F e / H]=0.196-1.527 M_{K}+0.091 M_{K}^{2}+1.886\left(V-K_{S}\right)-0.142\left(V-K_{S}\right)^{2}
$$

onde $K_{S}$ é a magnitude aparente $\mathrm{K}$ e $M_{K}$ é a magnitude $K$ absoluta. A segunda calibração obtida por eles baseou-se nas relações de massa-luminosidade de Delfosse et al. (2000) para bandas $\mathrm{V}$ e $K_{S}$. O resultado segue abaixo.

$$
[F e / H]=-0.149-6.508 \Delta M_{K}, \Delta M=M_{a s s}-M a s s_{K} .
$$

\section{(b) Johnson \& Apps (2009):}

Segundo esses autores, a metalicidade pode ser inferida através da relação:

$$
[F e / H]=0.56 \Delta M_{K}-0.05, \Delta M_{K}=M S-M_{K}
$$

onde $\Delta M_{K}$ é a diferença entre a média de $[\mathrm{Fe} / \mathrm{H}]$ de estrelas FGK de sequência principal do catálogo de Valenti \& Fischer (2005), definido como o polinômio de quinta ordem 
$M S=\Sigma a_{i}\left(V-K_{S}\right)^{i}$, onde a $=-9.58933,17.3952,2.22598,-0.258854,0.0113399$, e a magnitude absoluta da banda $K_{s}$.

\section{(c) Schalauffman \& Laughlin (2010):}

A calibração dos autores acima resultou na seguinte relação de metalicidade:

$$
[F e / H]=0.79 \Delta\left(V-K_{S}\right)-0.17,\left(V-K_{S}\right)=\Delta\left(V-K_{S}\right)_{o b s}-\left(V-K_{S}\right)_{i s o}
$$

onde $\left(V-K_{S}\right)_{o b s}$ é a cor $V-K_{S}$ observada e $\left(V-K_{S}\right)_{i s o}$ é uma função polinomial de quinta ordem de $M_{K_{S}}$ que descreve a sequência principal da vizinhança solar a partir do catálogo de Valenti \& Fischer (2005).

\section{(d) Neves et al. (2012):}

Esses autores também se basearam nas diferenças entre as cores $\left(V-K_{S}\right)$ observadas e aquelas descritas para a vizinhança solar de acordo com o catálogo de Valenti \& Fischer (2005). A calibração obtida por eles foi:

$$
[F e / H]=0.57 \Delta\left(V-K_{S}\right)-0.17
$$

Infelizmente, não pudemos testar nenhuma dessas calibrações neste trabalho, pois todas utilizam magnitudes absolutas e não possuimos medidas de paralaxe para nossa amostra.

\subsection{Calibrações espectroscópicas}

\subsubsection{Força das bandas moleculares}

O sistema atualmente usado para classificação de subanãs M é baseado na força das bandas moleculares $\mathrm{TiO}$ e $\mathrm{CaH}$ próximas a $7000 \AA$, quantificadas através dos índices espectrais CaH2, CaH3 e TiO5, como definidos por Reid et al. (1995):

$$
R_{\text {int }}=\frac{F_{W}}{F_{\text {cont }}}
$$

onde $F_{\text {cont }}$ é o fluxo do pseudo-contínuo definido no intervalo entre $7042 \AA$ e $7046 \AA$, e $F_{W}$ é o fluxo médio nos intervalos definidos para cada banda como mostra a Tabela (3.1). Os 
Tabela 3.1 - Índices espectrais originalmente definidos por Reid et al. (1995). Os índices são calculados a partir da razão do fluxo médio ao longo dos intervalos de comprimento de onda especificados.

\begin{tabular}{ccc}
\hline Nome do índice & $F_{W}(\AA)$ & $F_{\text {cont }}(\AA)$ \\
\hline TiO1 & $6718-6723$ & $7042-7046$ \\
TiO2 & $7058-7061$ & $7042-7046$ \\
TiO3 & $7092-7097$ & $7042-7046$ \\
TiO4 & $7130-7135$ & $7042-7046$ \\
TiO5 & $7126-7135$ & $7042-7046$ \\
$\mathrm{CaH} 1$ & $6380-6390$ & $7042-7046$ \\
$\mathrm{CaH} 2$ & $6814-6846$ & $7042-7046$ \\
$\mathrm{CaH} 3$ & $6960-6990$ & $7042-7046$ \\
$\mathrm{CaOH}$ & $6230-6240$ & $7042-7046$ \\
$\mathrm{H} \alpha$ & $6560-6566$ & $7042-7046$ \\
\hline
\end{tabular}

índices CaH2 e CaH3 medem o nível do fluxo médio da principal banda de CaH em relação ao ponto de referência em $7044 \AA$ (que define o pseudo-contínuo local). O índice TiO5 mede o nível do fluxo médio em uma região dentro das bandas de $\mathrm{TiO}$ em relação ao mesmo pseudo-contínuo.

O índice CaH2 correlaciona-se com a temperatura das anãs-M, enquanto o índice TiO5 varia tanto com a temperatura quanto com a metalicidade (Lépine et al. 2007).

Reid et al. (1995) calibraram também uma relação (equação 3.7) entre o índice [TiO5] e o tipo espectral para anãs válida no intervalo espectral entre K7 e M6.5:

$$
\text { tipo espectral }=-10.775[\text { TiO5 }]+8.2, \quad \sigma= \pm 0.5 \text { classe } .
$$

A amostra para calibração de Reid et al. (1995) continha 88 anãs frias a uma distância $r<8 p c$ que foram espectroscopicamente classificadas por Henry et al (1994) e Kirkpatrick et al. (1995).

\subsubsection{O índice de metalicidade $\zeta_{\mathrm{TiO} / \mathrm{CaH}}$}

A seleção cinemática (seção 1.3.4) de anãs do disco (com $[\mathrm{Fe} / \mathrm{H}] \sim 0.0$ ) feita por Lépine et al. (2007) permitiu calibrar uma correlação entre o índice TiO5 e a soma dos índices CaH2 e CaH3 para essas estrelas. O método se baseia em analisar a relação existente entre 
[TiO5] e [CaH2]+[CaH3] para estrelas do disco e do halo galáctico. A Figura (3.1), obtida de Lépine et al. (2007) para anãs M, mostra como estrelas do disco se agrupam em uma estreita faixa no centro do gráfico, enquanto as estrelas do halo aparecem mais dispersas. Lépine et al. (2007) calibraram uma relação entre [TiO5] e [CaH2]+[CaH3] para as estrelas
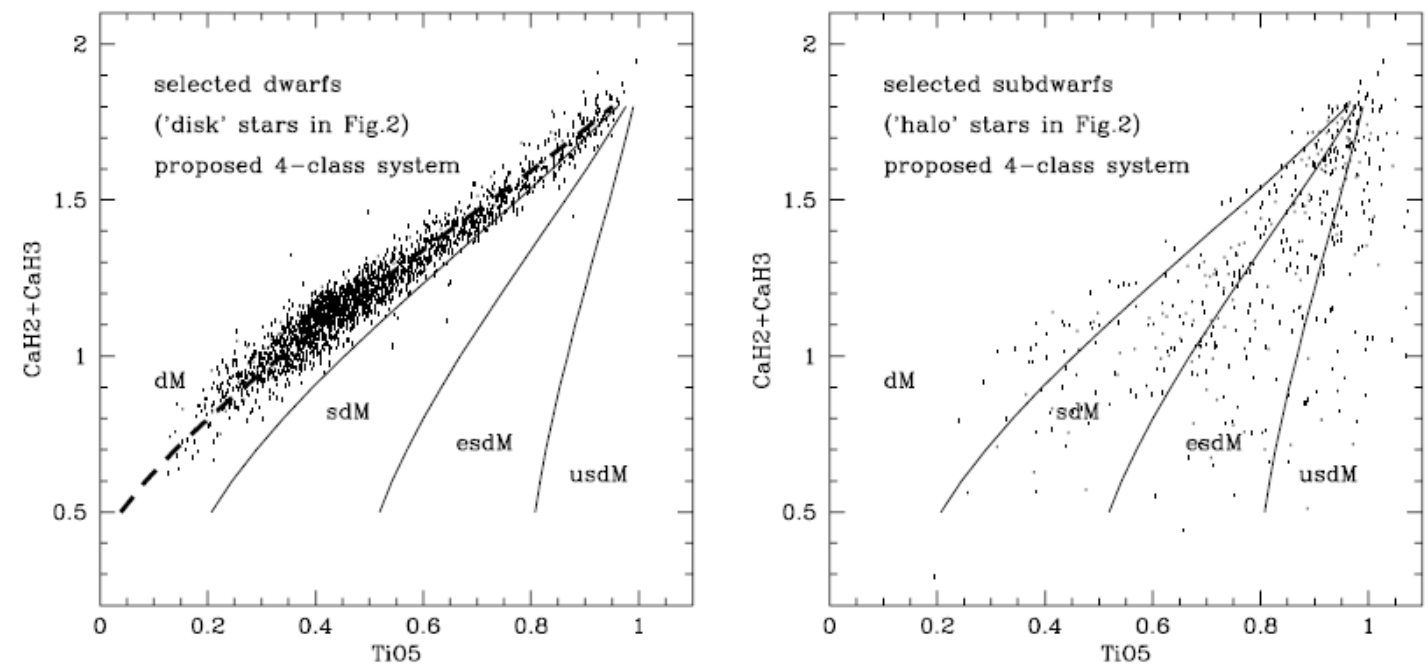

Figura 3.1: $[\mathrm{CaH} 2]+[\mathrm{CaH} 3]$ versus $[\mathrm{TiO} 5]$ para estrelas do disco e do halo para a amostra de Lépine et al. (2007)

do disco mostrada abaixo:

$$
[\mathrm{TiO} 5]_{Z_{\odot}}=-0.164(\mathrm{CaH})^{3}+0.670(\mathrm{CaH})^{2}-0.118(\mathrm{CaH})-0.050
$$

onde $(\mathrm{CaH})=[\mathrm{CaH} 2]+[\mathrm{CaH} 3]$. Esta relação, no entanto, foi refinada por Dithal et al. (2012) e escrita como:

$$
[\mathrm{TiO} 5]_{Z_{\odot}}=-0.047-0.127(\mathrm{CaH})+0.694(\mathrm{CaH})^{2}-0.183(\mathrm{CaH})^{3}-0.005(\mathrm{CaH})^{4} .
$$

A diferença entre as duas expressões está no tratamento de estrelas de tipo $\mathrm{K}$ tardia ("late") e M-jovem ("early"). Entretanto, o trabalho de Lépine et al. (2012) mostrou que a equação (3.8) superestima a metalicidade para os subtipos mais jovens ("earlier"), enquanto a equação (3.9) subestima a metalicidade para estes mesmos subtipos espectrais. A relação foi novamente reconsiderada por Lépine et al. (2012) passando a ser:

$$
[\mathrm{TiO} 5]_{Z_{\odot}}=0.622-1.906(\mathrm{CaH})+2.211(\mathrm{CaH})^{2}-0.588(\mathrm{CaH})^{3}
$$

De acordo com essas relações, estrelas do disco apresentam $\mathrm{TiO} 5 \simeq[\mathrm{TiO} 5]_{Z_{\odot}}$, enquanto estrelas do halo se espalham entre valores de $[\mathrm{TiO} 5]_{Z_{\odot}} \lesssim \mathrm{TiO} 5 \lesssim 1$. A Figura (3.2) de 
Woolf et al. (2009) mostra como estrelas mais pobres em metais se afastam da curva (formada por uma diagonal central) caracterizada pelas estrelas do disco ricas em metais. Baseando-se nesta ideia, Lépine et al. (2007) construiram um índice para mensurar a

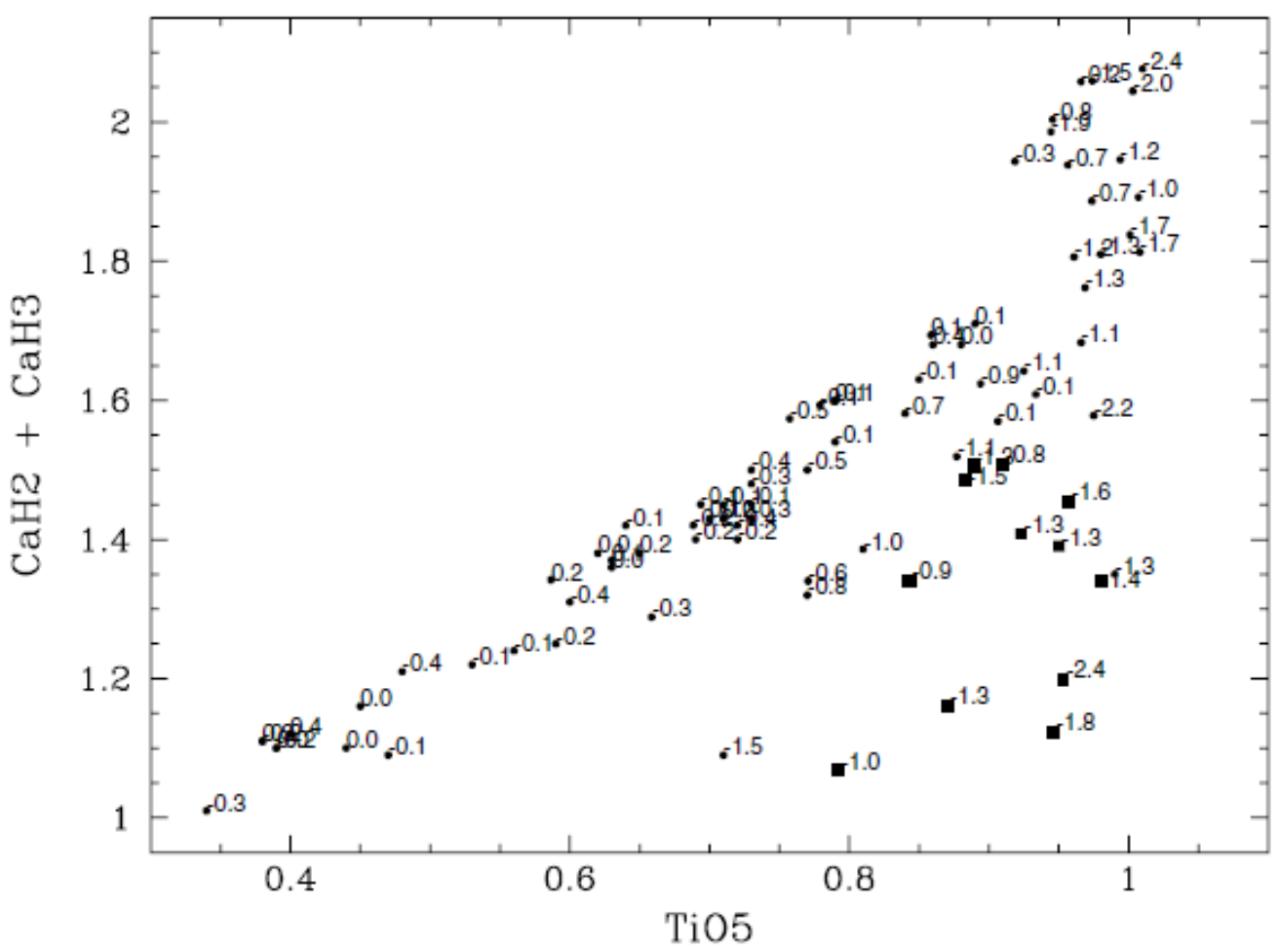

Figura 3.2: $[\mathrm{CaH} 2]+[\mathrm{CaH} 3]$ versus $[\mathrm{TiO} 5]$ do trabalho de Woolf et al. 2009. Números próximos aos pontos indicam estrelas com seus respectivos valores de $[\mathrm{Fe} / \mathrm{H}]$. Círculos cheios são estrelas do trabalho de Woolf \& Wallerstein (2006) e quadrados cheios são estrelas do trabalho de Woolf et al. (2009).

distância da curva definida por estrelas do disco de determinada estrela. Este índice, chamado $\zeta_{\mathrm{TiO} / \mathrm{CaH}}$, baseia-se na força relativa entre as bandas de $\mathrm{TiO}$ e $\mathrm{CaH}$ expressa em relação à banda de $\mathrm{TiO}$ calibrada para estrelas do disco (equações 3.8, 3.9, 3.10), sendo definido como:

$$
\zeta_{T i O / C a H}=\frac{1-T i O 5}{1-[T i O 5]_{Z_{\odot}}},
$$

onde $[\mathrm{TiO} 5]_{Z_{\odot}}$ é dada pelas equações (3.8, 3.9 ou 3.10). O parâmetro $\zeta_{\mathrm{TiO} / \mathrm{CaH}}$ pode, então, ser calculado para qualquer estrela com base nos índices TiO5, CaH2 e CaH3. Todas as estrelas com metalicidade igual ao do disco galáctico apresentarão $\zeta_{\mathrm{TiO} / \mathrm{CaH}}=1$, enquanto estrelas com metalicidade maior que a do disco apresentarão $\zeta_{\mathrm{TiO} / \mathrm{CaH}}>1$ e estrelas com metalicidade menor que a do disco, $\zeta_{\mathrm{TiO} / \mathrm{CaH}}<1$. 
Lépine et al. (2007) testaram a consistência do índice $\zeta_{T i O / C a H}$ como um indicador de metalicidade comparando o valor obtido de $\zeta_{\mathrm{TiO} / \mathrm{CaH}}$ entre componentes de sistemas binários resolvidos (onde espera-se que as duas estrelas compartilhem as mesmas abundâncias) no intervalo espectral K5-M9. O resultado obtido por eles está apresentado na Figura (3.3). Pode-se notar que os valores de $\zeta_{\mathrm{TiO} / \mathrm{CaH}}$ para as componentes estão fortemente correlacionados; isto somente é possível se as componentes compartilham a mesma metalicidade e se o parâmetro $\zeta_{\mathrm{TiO} / \mathrm{CaH}}$ realmente for uma medida consistente de metalicidade para estrelas de baixa massa.

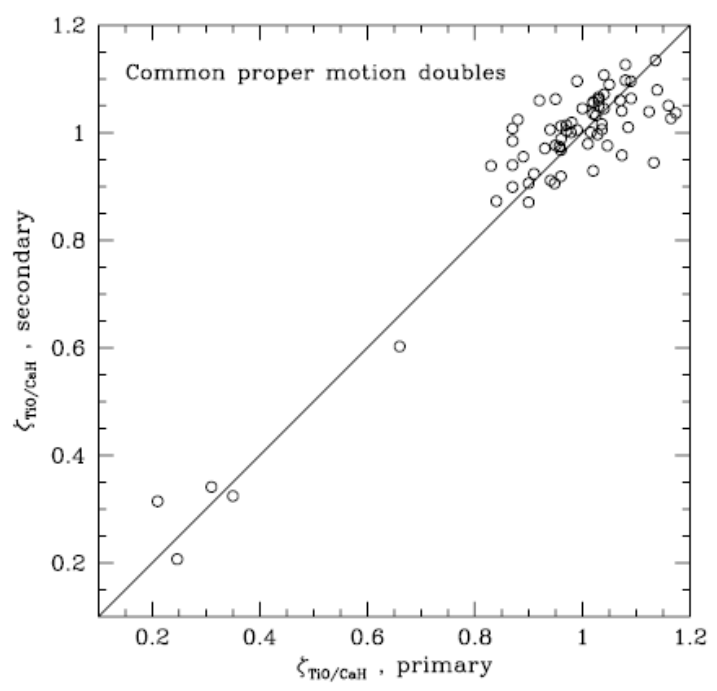

Figura 3.3: Comparação entre o índice de metalicidade $\zeta_{\mathrm{TiO} / \mathrm{CaH}}$ para componentes de sistemas binários resolvidos (de Lépine et al. 2007).

Este trabalho baseia-se justamente em avaliar o índice $\zeta_{\mathrm{TiO} / \mathrm{CaH}}$ como um indicador de metalicidade para a amostra de estrelas do hemisfério sul. Nossos resultados são apresentados no capítulo (4).

\subsubsection{Subclasses de metalicidade}

Além de classificar as estrelas em pobres ou ricas em metais pode-se considerar uma dessas classes e subdividi-la em intervalos menores de metalicidade, isto é, em subclasses de metalicidade. O trabalho de agrupar anãs M pobres em metais em subclasses foi iniciado por Gizis et al. (1997). A seguir, apresenta-se os trabalhos mais importantes sobre esse tema. 


\section{(a) Gizis et al. (1997): G97}

Como já apresentado na seção (1.3.3), Gizis et al. (1997) foram os primeiros a propor uma classificação de metalicidades para anãs e subanãs M. A classificação de G97 dividia anãs $M$ em três subclasses de metalicidade: as dM que são anãs $M$ de metalicidade aproximadamente solar, as sdM que são subanãs M podres em metais e as esdM que são subanãs M extremamente pobres em metais. Entretanto, nenhuma equação para estimar a metalicidades dessas estrelas foi calibrada. O sistema de G97 foi inicialmente definido para sub-tipos espectrais mais jovens que sdM5/esdM5, sendo posteriormente expandido para os objetos mais frios (Schweitzer et al. 1999; Lépine et al. 2003c, 2004; Scholz et al. 2004) chegando até a subanãs de tipo espectral L (Lépine et al. 2003b; Burgasser et al. 2003; Gizis \& Harvin 2006).

A classificação de G97 era feita somente com base na força das bandas de CaH1, CaH2, CaH3 com relação a força da banda de TiO5, analisando-se separadamente os diagramas de [CaH1] versus [TiO5], [CaH2] versus [TiO5] e [CaH3] versus [TiO5], como mostrado na Figura (3.4). Contudo, a partir do trabalho de Lépine et al. (2003c) tornou-se comum representar as sdM confirmadas espectroscopicamentes em um único diagrama de [CaH2]+[CaH3] versus [TiO5] (Lépine et al. 2004; Scholz et al. 2004a; Reid \& Gizis 2005), conforme mostrado na Figura (3.1).

Uma simplificação do sistema de G97 foi proposta por Burgasser \& Kirkpatrick (2006) usando somente duas relações para definir a separação entre dM, sdM e esdM no diagrama $[\mathrm{CaH} 2]+[\mathrm{CaH} 3]$ versus [TiO5]. Estes separadores foram validados utilizando objetos previamente classificados, sendo essencialmente equivalente ao sistema original de G97.

\section{(b) Lépine et al. (2007)}

Em 2007, Lépine et al. propuseram-se a re-examinar o sistema de classificação de metalicidades para estrelas anãs e subanãs vermelhas sob a luz de novas medidas espectroscópicas de 1983 estrelas com altos movimentos próprios obtidas do catálogo LSPM-north (Lépine \& Shara 2005). Através de observações de estrelas duplas com movimentos próprios comuns, eles redefiniram as subclasses de metalicidade baseados na calibração empírica da razão entre $\mathrm{TiO} / \mathrm{CaH}$ para estrelas de metalicidade aproximadamente solar como discutido 

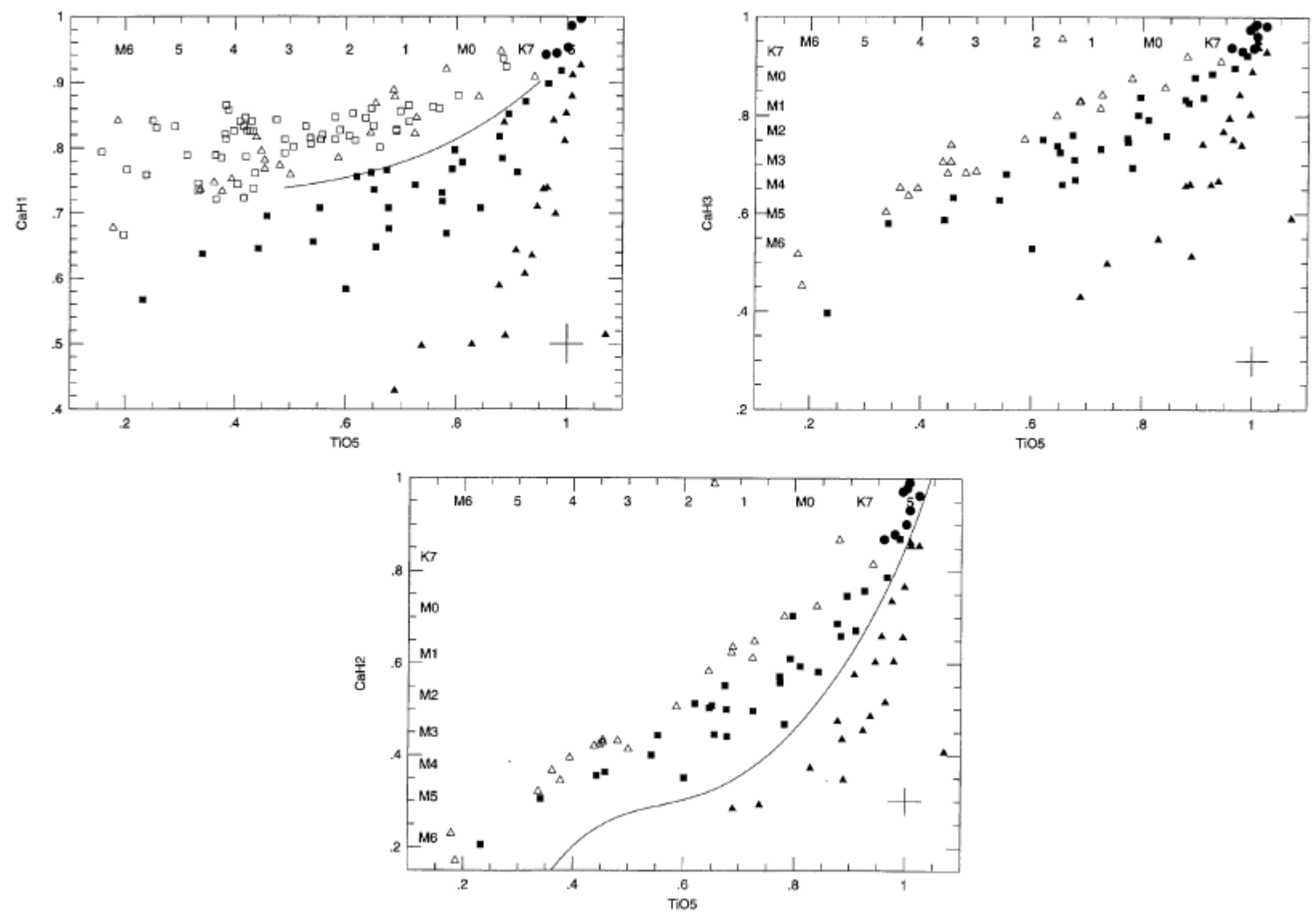

Figura 3.4: Classificação de subanãs em subclasses de metalicidade de acordo com G97. sdM aparecem como quadrados cheios, esdM como triângulos cheios, sdK como círculos cheios e estrelas não classificadas como subanãs aparecem como círculos abertos. A barra de erro de \pm 0.03 aparece no canto inferior direito.

na seção (3.2.2).

Com a definição do índice $\zeta_{T i O / C a H}$, eles reclassificaram o sistema de G97 introduzindo uma nova subclasse, as usdK e usdM, subanãs ultra pobres em metais. Mas o novo sistema de Lépine et al. (2007) não se limita a apenas introduzir uma nova subclasse para abranger estrelas mais pobres em metais. Ao mostrar que $\zeta_{\mathrm{TiO} / \mathrm{CaH}}$ poderia ser um indicador consistente de metalicidade (Figura 3.3), eles mostraram que na classificação de G97, estrelas de sistemas binários resolvidos que devem compartilhar a mesma metalicidade pertenciam a subclasses diferentes, ou seja, os contornos de isometalicidades não eram seguidos adequadamente no plano $[\mathrm{CaH} 2]+[\mathrm{CaH} 3]$ versus [TiO5] (Figura 3.5). A partir daí, eles redefiniram novas classes para que essas estrelas pertencessem às mesmas subclasses de metalicidade. Consequentemente, os contornos de isometalicidades respeita- 
ram bem melhor os limites de metalicidade (Figura 3.5). Assim, o sistema de Lépine et al. (2007) conta com quatro subclasses de metalicidades para anãs K e M: dK/dM, sdK/sdM, esdK/esdM e usdK/usdM.
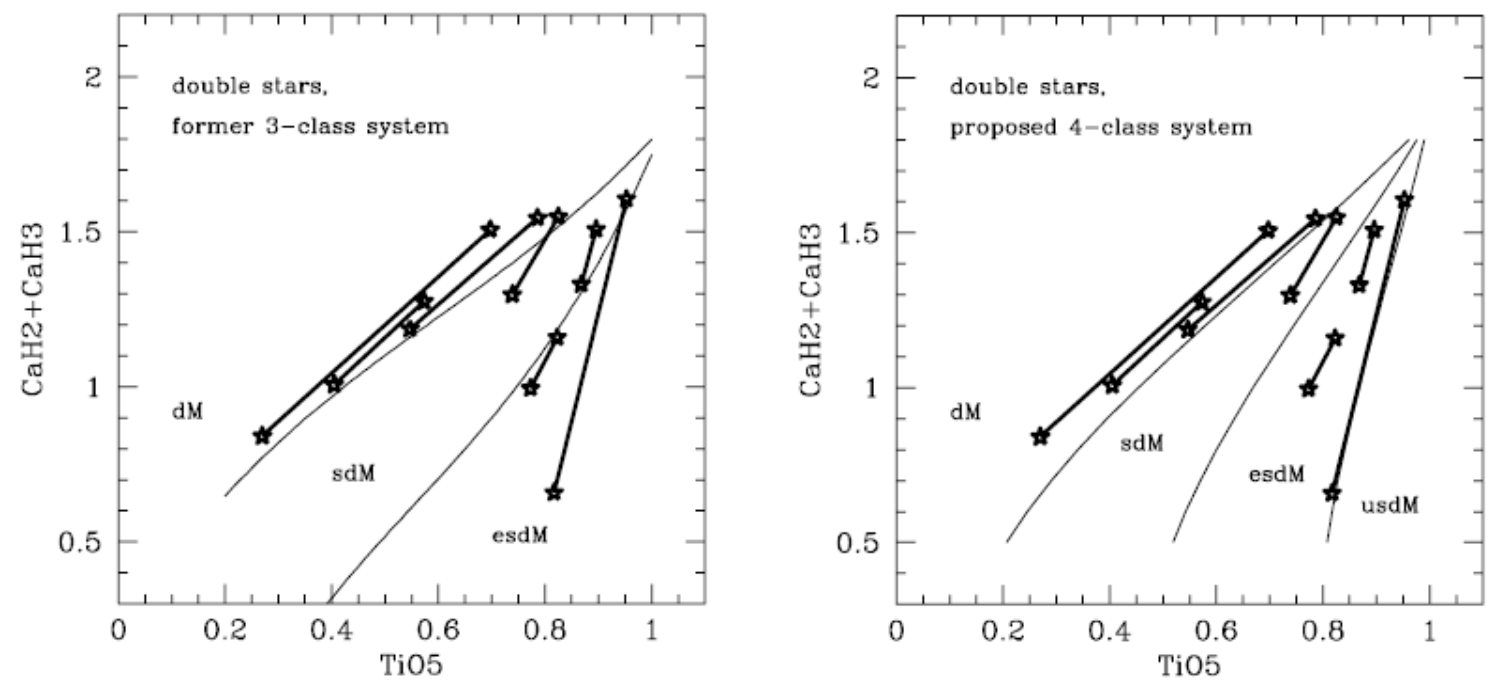

Figura 3.5: Distribuição dos valores dos índices de TiO5 e CaH2+CaH3 para pares de movimento próprio comum. As primárias e secundárias são conectadas por linhas retas. No antigo sistema de classificação (esquerda), alguns pares atravessavam os separadores de subclasses de forma que os membros do sistema apresentavam diferentes metalicidades. O novo sistema (direita) (Lépine et al. 2007) assegura que primária e secundária se localizem na mesma subclasse, que é o que se espera de pares que tiveram a mesma origem.

\section{(c) Woolf et al. (2009)}

Em 2009, Woolf et al. analisaram uma amostra de 12 sdM e esdM com espectroscopia em alta resolução. Calibraram, pela primeira vez, uma equação para $[\mathrm{Fe} / \mathrm{H}]$ em função de $\zeta_{\mathrm{TiO} / \mathrm{CaH}}$ para estimar as metalicidades dos objetos, com precisão de \pm 0.3 dex para estrelas com $[\mathrm{Fe} / \mathrm{H}]$ no intervalo de -1.5 dex a +0.05 dex e temperatura entre $3500 \mathrm{~K}$ a 4000K. A equação abaixo mostra a calibração obtida por eles:

$$
[\mathrm{Fe} / \mathrm{H}]=-1.685+1.632 \zeta_{\mathrm{tiO} / \mathrm{CaH}}
$$

A Figura (3.6) apresenta o ajuste para a amostra que inclui as 12 estrelas para calibração e outras com $[\mathrm{Fe} / \mathrm{H}]$ previamente conhecidos. De acordo com a calibração de Woolf et al. (2009), as subclasses de metalicidades definidas por Lépine et al. (2007) se encaixam no intervalos de metalicidades apresentados na Tabela (3.2). 


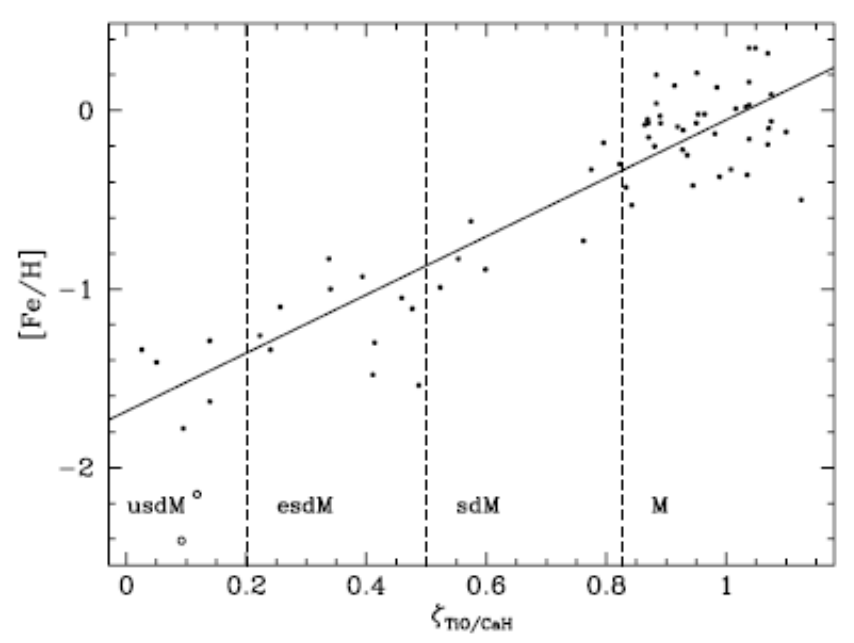

Figura 3.6: Calibração de Woolf et al. (2009) para uma amostra de sdM e esdM observada em alta resolução. Os pontos são as estrelas e a linha sólida o ajuste linear. $[\mathrm{Fe} / \mathrm{H}]=$ $a+b \zeta_{\text {TiO/CaH }} \operatorname{com} a=-1.685 \pm 0.079$ e $b=1.632 \pm 0.096$.

Tabela 3.2 - Divisão em subclasses de metalicidades como apresentada em Woolf et al.(2009) e em acordo com as quatro subclasses propostas por Lépine et al. (2007).

\begin{tabular}{ccc}
\hline Tipo espectral & $\zeta_{\mathrm{TiO} / \mathrm{CaH}}$ & {$[\mathrm{Fe} / \mathrm{H}]$} \\
\hline $\mathrm{M}$ & $>0.825$ & $>-0.34$ \\
$\mathrm{sdM}$ & 0.500 a 0.825 & $-0.87 \mathrm{a}-0.34$ \\
esdM & 0.200 a 0.500 & $-1.36 \mathrm{a}-0.87$ \\
usdM & $<0.200$ & $<-1.36$ \\
\hline
\end{tabular}

\section{(d) Rojas-Ayala et al. (2010)}

Em 2010, Rojas-Ayala et al. publicaram uma nova técnica para medir a metalicidade de anãs M. A técnica é baseada apenas em medições de índices espectrais em resolução moderada $(R \sim 2700)$ na banda $\mathrm{K}$ do espectro eletromagnético, não necessitando nem de magnitudes na banda $\mathrm{V}$ nem de medidas de paralaxe, o que permite analisar estrelas mais fracas ou mais distantes. Nesse trabalho, eles analisaram 17 anãs M em sistemas binários com primárias $\mathrm{F} / \mathrm{G} / \mathrm{K}$ para que servissem como calibradores de metalicidade. Com medidas de larguras equivalentes do dubleto de NaI $(2206 \mu \mathrm{m}$ e $2209 \mu \mathrm{m})$, do tripleto de CaI ( $2261 \mu \mathrm{m}, 2263 \mu \mathrm{m}$ e $2265 \mu \mathrm{m})$ e com o índice espectral de absorção de água sensível a temperatura estelar, eles construiram uma escala de metalicidade com coeficiente de correlação maior que $R^{2}=0.63$ (que foi o obtido por Schalauffman \& Laughlin 2010) e incerteza de 0.15dex. No entanto, a calibração de Rojas-Ayala et al. (2010) somente é válida para anãs M com metalicidade no intervalo de -0.5 dex a +0.5 dex. 
Como esta técnica trabalha apenas na banda $\mathrm{K}$, utilizando linhas em comprimentos de onda em torno de $2200 \mu \mathrm{m}$, não foi possível testá-la a nossa amostra de anãs M em questão, pois ela consiste apenas de espectros na banda $\mathrm{V}$ (entre $0.6 \mu \mathrm{m}$ a $0.9 \mu \mathrm{m}$ aproximadamente).

Pode-se notar que houve grandes esforços para separar e calibrar a metalicidade das estrelas subanãs M ao longo dos anos e que, ainda assim, é necessário um trabalho de refinamento para cada um desses trabalhos. O presente trabalho é focado na calibração de metalicidade através do índice $\zeta_{\mathrm{TiO} / \mathrm{CaH}}$ definido em Lépine et al. (2007) e na comparação com a calibração obtida por Woolf et al. (2009). 
Capítulo 4

\section{Resultados e discussões}

No capítulo anterior, discutiu-se as calibração de metalicidade presentes na literatura para anãs M. Mostrou-se que ao longo dos anos houve importantes avanços neste estudo, tanto com publicações de novas calibrações quanto com refinamentos das já existentes. Neste capítulo analisa-se a capacidade de $\zeta_{\mathrm{TiO} / \mathrm{CaH}}$ atuar como um medidor de metalicidade.

Na Tabela (4.1) apresenta-se os valores para os índices espectroscópicos e as abundâncias $[\mathrm{Fe} / \mathrm{H}]$ e $[\mathrm{C} / \mathrm{Fe}]$. Uma tabela com as abundâncias para toda a amostra observada pode ser encontrada no Apêndice (A), enquanto todos os espectros obtidos e reduzidos podem visualizados no Apêndice (B). As abundâncias foram obtidas pelo n-sspp através das respectivas companheiras primárias. O erro tanto para $[\mathrm{Fe} / \mathrm{H}]$ como para $[\mathrm{C} / \mathrm{Fe}]$ é de 0.2 dex. Como discutido anteriormente, somente estão presentes na Tabela (4.1) as estrelas secundárias com razão $\mathrm{S} / \mathrm{N} \geq 20$. 
Tabela 4.1 - Índices espectroscópicos e metalicidades para as anãs M. A incerteza nas abundâncias é de 0.2 dex.

\begin{tabular}{|c|c|c|c|c|c|c|c|c|}
\hline \multicolumn{2}{|c|}{ Anãs M } & \multicolumn{5}{|c|}{ Índices espectroscópicos } & \multicolumn{2}{|c|}{ Abundâncias } \\
\hline Identificação & Nome & {$[\mathrm{CaH} 2]+[\mathrm{CaH} 3]$} & [TiO5] & $\zeta_{\mathrm{TiO} / \mathrm{CaH}}(\mathrm{L} 07)^{*}$ & $\zeta_{\mathrm{TiO} / \mathrm{CaH}}(\mathrm{D} 12)^{*}$ & $\zeta_{\mathrm{TiO} / \mathrm{CaH}}(\mathrm{L} 12)^{*}$ & {$[\mathrm{Fe} / \mathrm{H}]$} & {$[\mathrm{C} / \mathrm{Fe}]$} \\
\hline 2 & PM_I01008-2126 & 1.38 & 0.65 & 0.96 & 0.88 & 1.03 & -0.92 & 0.81 \\
\hline 3 & PM_I00426-2314 & 1.52 & 0.66 & 1.30 & 1.10 & 1.44 & -0.88 & 0.75 \\
\hline 4 & PM_I01266-4842E & 1.10 & 0.36 & 1.08 & 1.06 & 1.09 & -1.37 & 1.21 \\
\hline 5 & PM_I01543-2249N & 1.16 & 0.42 & 1.07 & 1.04 & 1.09 & -0.61 & 0.66 \\
\hline 6 & PM_I02300-2402E & 1.52 & 0.74 & 1.00 & 0.84 & 1.11 & 0.32 & -0.48 \\
\hline 7 & PM_I02462-2311 & 1.78 & 0.83 & 2.70 & 1.07 & 2.05 & -0.45 & 0.35 \\
\hline 8 & PM_I02542-6619 & 1.25 & 0.48 & 1.10 & 1.06 & 1.15 & -0.57 & 1.09 \\
\hline 9 & PM_I04325-5657S & 1.42 & 0.73 & 0.81 & 0.73 & 0.87 & -0.92 & 0.79 \\
\hline 10 & $\mathrm{PM}_{-} \mathrm{I} 04477-3044 \mathrm{E}$ & 1.06 & 0.52 & 0.78 & 0.77 & 0.78 & -0.84 & 0.80 \\
\hline 12 & PM_I05425-4305W & 1.17 & 0.40 & 1.12 & 1.09 & 1.14 & -0.58 & 0.45 \\
\hline 13 & PM_I05442-2417N & 1.17 & 0.48 & 0.97 & 0.95 & 0.99 & -0.56 & 0.43 \\
\hline 14 & PM_I05560-4511 & 1.08 & 0.38 & 1.04 & 1.02 & 1.04 & -0.16 & 0.11 \\
\hline 15 & PM_I06276-2356 & 1.37 & 0.62 & 1.00 & 0.93 & 1.07 & -0.22 & 0.16 \\
\hline 16 & PM_I08239-7549E & 0.98 & 0.34 & 0.98 & 0.97 & 0.98 & -0.29 & 0.20 \\
\hline 17 & PM_I08386-3857 & 1.23 & 0.49 & 1.04 & 1.00 & 1.07 & -0.75 & 0.63 \\
\hline 18 & PM_I13133-4153S & 1.18 & 0.60 & 0.76 & 0.74 & 0.77 & -1.40 & 1.24 \\
\hline 19 & PM_I18241-5107 & 1.08 & 0.40 & 1.00 & 0.98 & 1.01 & -0.54 & 0.83 \\
\hline 19 & PM_I18241-5107 & 1.08 & 0.40 & 1.00 & 0.98 & 1.01 & -0.33 & 0.22 \\
\hline 21 & PM_I20371-5816 & 1.37 & 0.57 & 1.14 & 1.05 & 1.22 & -1.62 & 1.90 \\
\hline 22 & $\mathrm{PM}_{-} \mathrm{I} 21175-4142 \mathrm{~W}$ & 1.36 & 0.58 & 1.08 & 1.01 & 1.15 & -0.93 & 0.86 \\
\hline 25 & PM_I22487-5613E & 1.27 & 0.54 & 1.02 & 0.98 & 1.07 & -0.38 & 0.20 \\
\hline 26 & PM_I23034-5311 & 1.38 & 0.70 & 0.82 & 0.75 & 0.88 & -1.19 & 1.27 \\
\hline 27 & PM_I23099-2056 & 1.63 & 0.84 & 0.94 & 0.68 & 1.03 & -1.43 & 1.51 \\
\hline
\end{tabular}

* L07 é em referência a Lépine et al. (2007), D12 Dithal et al. (2012) e L12 Lépine et al. (2012). 


\subsection{Validação dos índices espectroscópicos}

Neste trabalho, chama-se validação ao ato de admitir que os cálculos para obtenção dos índices espectroscópicos foram executados corretamente através da comparação com outro trabalho. Para isso, compara-se a relação entre os índices [CaH2] e [CaH2]+[CaH3] obtida para a amostra de anãs M deste estudo com a obtida por Woolf et al. (2009). O acordo entre elas está apresentada na Figura (4.1) e valida os cálculos para estes índices. Como os índices de TiO5 foram obtidos atráves da mesma metodologia que os índices de CaH2 e CaH3, pode-se considerar que os índices de TiO5 também foram calculados corretamente.

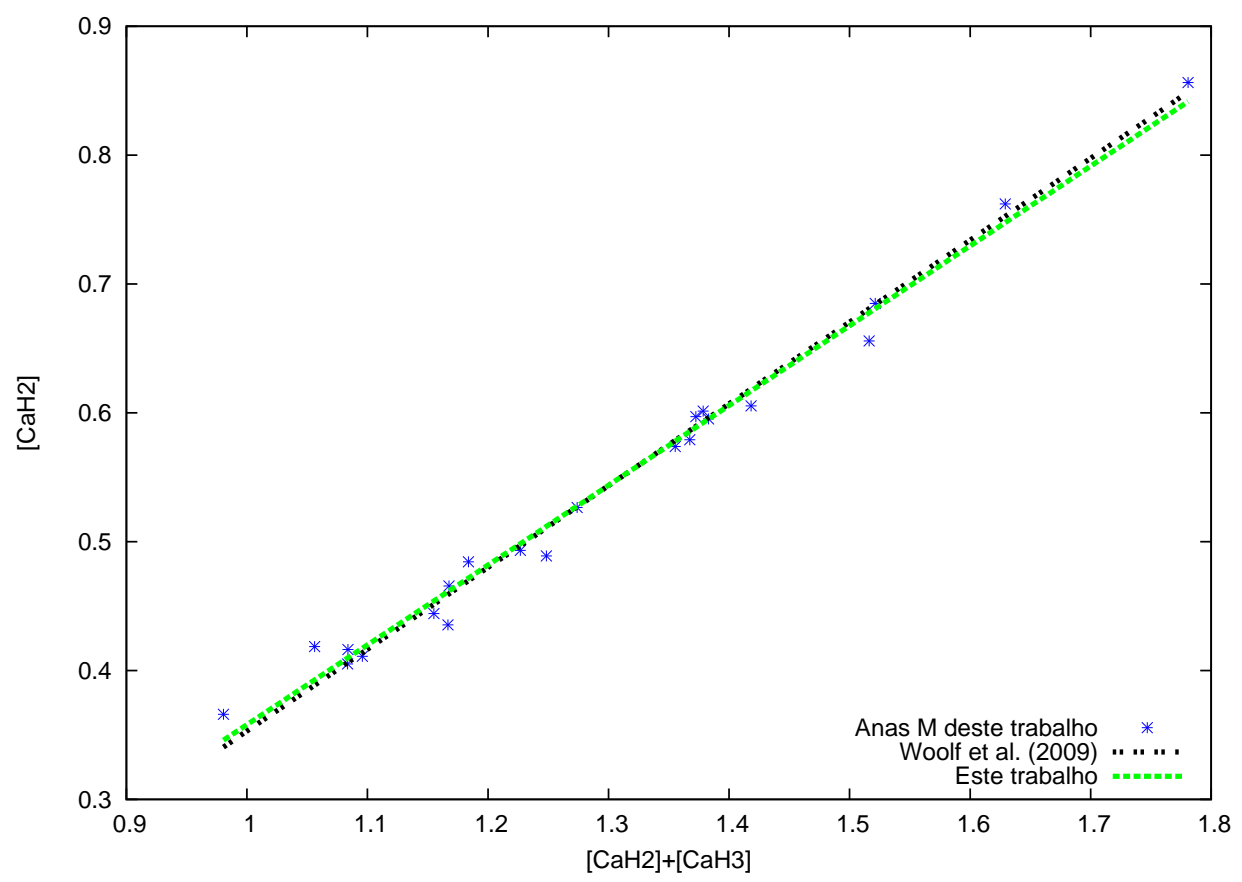

Figura 4.1: Comparação entre este trabalho e o de Woolf et al. (2009) para a relação entre $[\mathrm{CaH} 2]+[\mathrm{CaH} 3]$ e $[\mathrm{CaH} 2]$. A relação obtida por Woolf et al. (2009) foi $[\mathrm{CaH} 2]=$ $(-0.282 \pm 0.014)+(0.635 \pm 0.010)([\mathrm{CaH} 2]+[\mathrm{CaH} 3])$ e a obtida neste trabalho foi $[\mathrm{CaH} 2]=(-0.261 \pm 0.020)+(0.619 \pm 0.015)([\mathrm{CaH} 2]+[\mathrm{CaH} 3])$.

\subsection{A calibração em fluxo é necessária?}

A metodologia descrita por Reid et al. (1995) para o cálculo dos índices espectroscópicos utiliza a razão entre os fluxos médios absolutos nos intervalos especificados na Tabela (3.1). Tal metodologia é a mesma utilizada por Lépine et al. (2012) e neste trabalho. No entanto, nota-se que os índices calculados a partir dos espectros calibrados em fluxo são os mesmo 
que aqueles calculados a partir de espectros sem a calibração em fluxo, isto é, espectros em contagens versus comprimento de onda. A Figura (4.2) e a Tabela (4.2) mostram a comparação entre os índices [TiO5] e [CaH2]+[CaH3] obtidos com e sem a calibração em fluxo. As diferenças encontradas podem ser atribuidas à pequenas mudanças no perfil dos espectros causados pela calibração em fluxo. De fato, como os índices trabalham com razões de fluxos, sendo que o fluxo absoluto é diretamente proporcional às contagens, espera-se que os índices calculados das duas formas sejam iguais. Com isso, se detetou nenhuma razão para utilização da calibração em fluxo em trabalhos que dependem exclusivamente destes índices. Eliminando-se a calibração em fluxo da metologia, pode-se otimizar o tempo de observação que, de outra forma, seria parcialmente gasto para se observar as estrelas padrões espectrofotométricas, além de facilitar o processo de redução de dados. Mesmo assim, quando não indicado, utilizou-se os índices com a calibração em fluxo para manter a concordância com a metodologia de Lépine et al. (2012).

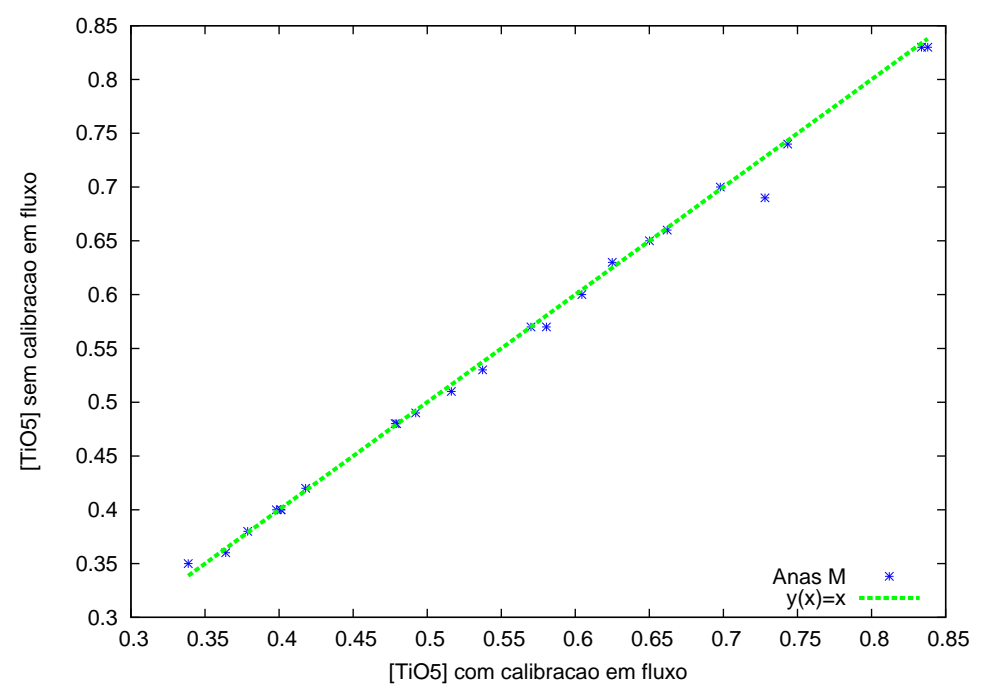

(a)

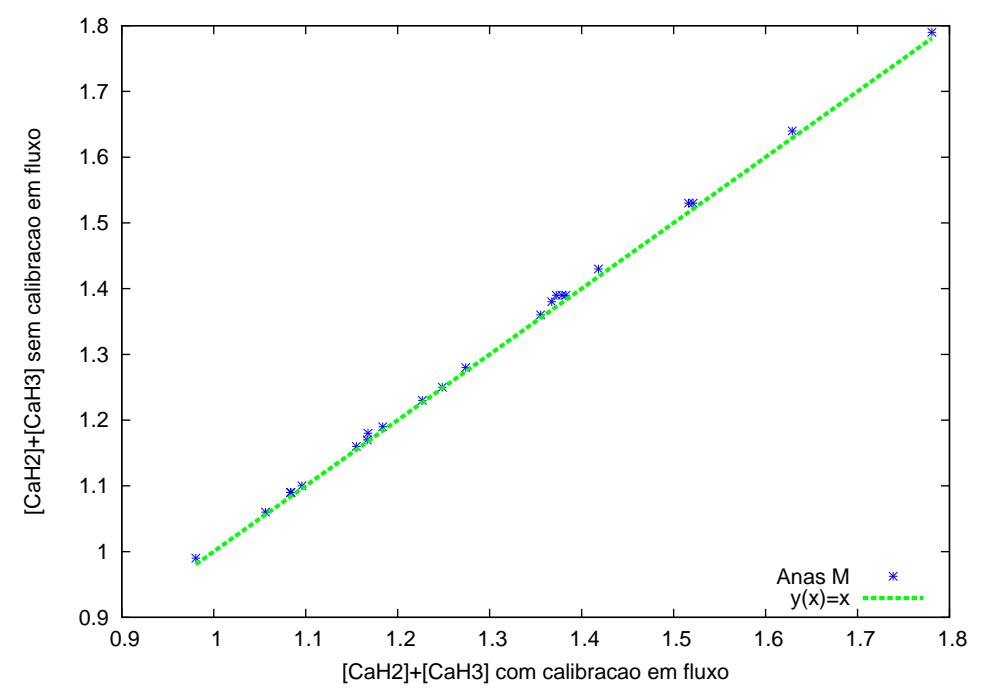

(b)

Figura 4.2: Efeito da calibração em fluxo: (a) Comparação entre o índice de TiO5 calculado com e sem calibração em fluxo. (b) Comparação entre a soma dos índices de $\mathrm{CaH} 2+\mathrm{CaH} 3$ calculado com e sem calibração em fluxo 
Tabela 4.2 - Comparação entre os índices espectroscópicos obtidos com e sem calibração em fluxo.

\begin{tabular}{|c|c|c|c|c|c|c|c|c|c|c|}
\hline \multicolumn{2}{|r|}{ Anãs M } & \multicolumn{3}{|c|}{ Com calibração em fluxo } & \multicolumn{3}{|c|}{ Sem calibração em fluxo } & \multicolumn{3}{|c|}{ Diferença } \\
\hline Identificação & Nome & {$[\mathrm{CaH} 2]+[\mathrm{CaH} 3]$} & [TiO5] & $\zeta_{\mathrm{TiO} / \mathrm{CaH}}(\mathrm{L} 12)$ & {$[\mathrm{CaH} 2]+[\mathrm{CaH} 3]$} & [TiO5] & $\zeta_{\mathrm{TiO} / \mathrm{CaH}}(\mathrm{L} 12)$ & {$[\mathrm{CaH} 2]+[\mathrm{CaH} 3]$} & [TiO5] & $\zeta_{\mathrm{TiO} / \mathrm{CaH}}$ \\
\hline 2 & PM_I01008-2126 & 1.52 & 0.66 & 1.44 & 1.53 & 0.66 & 1.54 & -0.02 & 0.00 & -0.10 \\
\hline 3 & PM_I00426-2314 & 1.38 & 0.65 & 1.03 & 1.39 & 0.65 & 1.06 & -0.01 & 0.00 & -0.03 \\
\hline 4 & $\mathrm{PM}_{-} \mathrm{I} 01266-4842 \mathrm{E}$ & 1.10 & 0.36 & 1.09 & 1.10 & 0.36 & 1.10 & 0.00 & 0.00 & -0.01 \\
\hline 5 & $\mathrm{PM}_{-} \mathrm{I} 01543-2249 \mathrm{~N}$ & 1.16 & 0.42 & 1.09 & 1.16 & 0.42 & 1.10 & 0.00 & 0.00 & -0.01 \\
\hline 6 & $\mathrm{PM}_{-} \mathrm{I} 02300-2402 \mathrm{E}$ & 1.52 & 0.74 & 1.11 & 1.53 & 0.74 & 1.16 & -0.01 & 0.00 & -0.05 \\
\hline 7 & PM_I02462-2311 & 1.78 & 0.83 & 2.05 & 1.79 & 0.83 & 2.25 & -0.01 & 0.01 & -0.21 \\
\hline 8 & PM_I02542-6619 & 1.25 & 0.48 & 1.15 & 1.25 & 0.48 & 1.16 & -0.01 & 0.00 & -0.02 \\
\hline 9 & $\mathrm{PM}_{-} \mathrm{I} 04325-5657 \mathrm{~S}$ & 1.42 & 0.73 & 0.87 & 1.43 & 0.69 & 1.02 & -0.01 & 0.03 & -0.15 \\
\hline 10 & PM_I04477-3044E & 1.06 & 0.52 & 0.78 & 1.06 & 0.51 & 0.79 & -0.01 & 0.00 & -0.01 \\
\hline 12 & PM_I05425-4305W & 1.17 & 0.40 & 1.14 & 1.17 & 0.40 & 1.16 & -0.01 & 0.00 & -0.01 \\
\hline 13 & PM_I05442-2417N & 1.17 & 0.48 & 0.99 & 1.18 & 0.48 & 1.01 & -0.01 & 0.00 & -0.02 \\
\hline 14 & PM_I05560-4511 & 1.08 & 0.38 & 1.04 & 1.09 & 0.38 & 1.05 & -0.01 & 0.00 & -0.01 \\
\hline 15 & PM_I06276-2356 & 1.37 & 0.62 & 1.07 & 1.39 & 0.63 & 1.11 & -0.02 & 0.00 & -0.04 \\
\hline 16 & $\mathrm{PM}_{-} \mathrm{I} 08239-7549 \mathrm{E}$ & 0.98 & 0.34 & 0.98 & 0.99 & 0.35 & 0.97 & -0.01 & -0.01 & 0.01 \\
\hline 17 & PM_I08386-3857 & 1.23 & 0.49 & 1.07 & 1.23 & 0.49 & 1.09 & -0.01 & 0.00 & -0.02 \\
\hline 18 & PM_I13133-4153S & 1.18 & 0.60 & 0.77 & 1.19 & 0.60 & 0.80 & -0.01 & 0.01 & -0.03 \\
\hline 19 & PM_I18241-5107 & 1.08 & 0.40 & 1.01 & 1.09 & 0.40 & 1.02 & -0.01 & 0.00 & -0.02 \\
\hline 21 & PM_I20371-5816 & 1.37 & 0.57 & 1.22 & 1.38 & 0.57 & 1.28 & -0.02 & 0.00 & -0.07 \\
\hline 22 & $\mathrm{PM}_{-} \mathrm{I} 21175-4142 \mathrm{~W}$ & 1.36 & 0.58 & 1.15 & 1.36 & 0.57 & 1.20 & -0.01 & 0.01 & -0.04 \\
\hline 25 & PM_I22487-5613E & 1.27 & 0.54 & 1.07 & 1.28 & 0.53 & 1.10 & -0.01 & 0.01 & -0.03 \\
\hline 26 & PM_I23034-5311 & 1.38 & 0.70 & 0.88 & 1.39 & 0.70 & 0.89 & -0.01 & 0.00 & -0.02 \\
\hline 27 & PM_I23099-2056 & 1.63 & 0.84 & 1.03 & 1.64 & 0.83 & 1.13 & -0.01 & 0.01 & -0.10 \\
\hline
\end{tabular}




\subsection{Efeito das franjas no cálculo dos índices espectroscópicos}

Como discutido no Capítulo 2, utilizou-se os procedimentos descritos em Goudfrooij et al (1998) para corrigir as franjas causadas pelo CCD nos espectros das anãs M. Para isso, a equipe de astrônomos residentes do SOAR forneceu rotinas automatizadas em Pyraf em fase de conclusão. No entanto, o procedimento não se mostrou eficiente para todas estrelas. A Figura (4.3) mostra um exemplo de um espectro que teve praticamente $100 \%$ de suas franjas eliminadas e a Figura (4.4) mostra outro cuja correção não surtiu nenhum efeito. A fim de determinar a influência das franjas para o cálculo dos índices, comparou-se os valores obtidos para $[\mathrm{CaH} 2]+[\mathrm{CaH} 3]$ e $[\mathrm{TiO} 5]$ utilizando-se os espectros com a correção das franjas e sem a correção. O resultado está apresentado na Tabela (4.3) e na Figura $(4.5)$.

A estrela PM_I00426-2314 (Figura 4.3) obteve o melhor resultado para a correção de franjas e a diferença entre os índices calculados com e sem a correção foi de 0.07 para [CaH2]+[CaH3] e 0.03 para o [TiO5]. Pode-se então, admitir que o erro cometido nos valores dos índices para os espectros cuja correção foi ineficiente não deve ultrapassar esses limites. Todas as diferenças mostradas na Tabela (4.3) são muito pequenas quando se leva em conta a razão $\mathrm{S} / \mathrm{N}$ média em torno de 25 desses espectros e a precisão do pipeline nsspp de 0.2 dex. Dessa forma, conclui-se que mesmo sem uma correção eficiente de franjas o erro acarretado não afeta a capacidade de $\zeta_{\mathrm{TiO} / \mathrm{CaH}}$ ser utilizado como um indicador de metalicidade para a amostra em questão.

A pequena influência da correção nos índices [CaH2], [CaH3] e [TiO5] é entendida pelo fato das franjas aparecerem predominante além de $7500 \AA$ e todos os índices serem calculados para comprimentos de onda inferiores a esse, de forma que para a região de interesse a correção pode ser desconsiderada. 


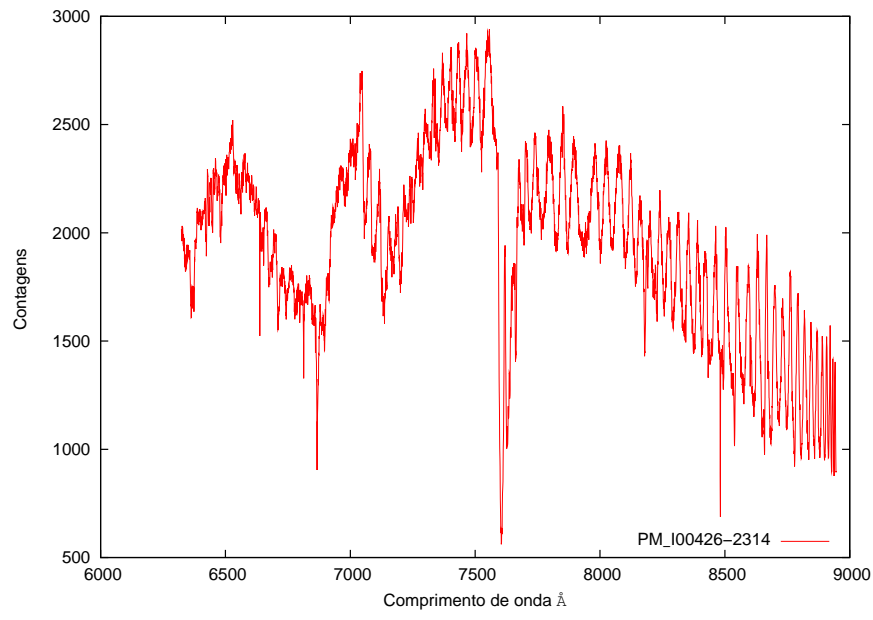

(a) Espectro sem correção de franjas

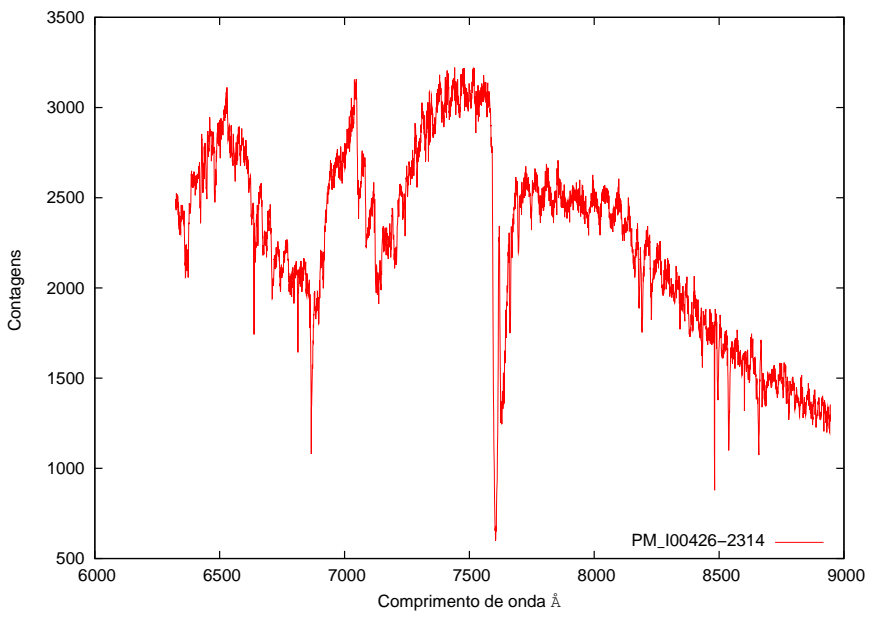

(b) Espectro com correção de franjas

Figura 4.3: Exemplo de espectro com correção de franjas eficiente.

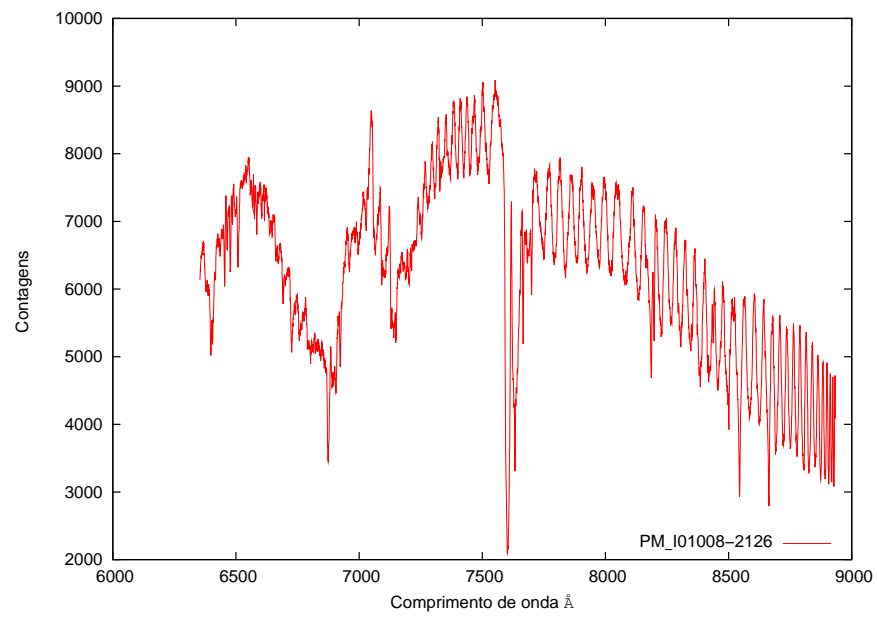

(a) Espectro sem correção de franjas

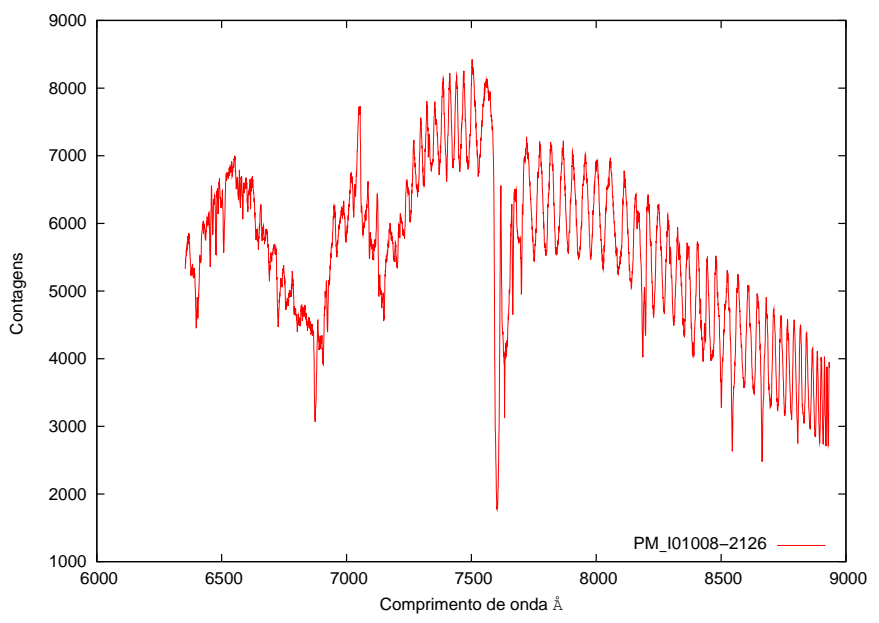

(b) Espectro com correção de franjas

Figura 4.4: Exemplo de espectro com correção de franjas ineficiente. 
Tabela 4.3 - Comparação entre os índices espectroscópicos obtidos com e sem correção de franjas.

\begin{tabular}{|c|c|c|c|c|c|c|c|c|c|c|}
\hline \multicolumn{2}{|c|}{ Anãs M } & \multicolumn{3}{|c|}{ Com correção de franjas } & \multicolumn{3}{|c|}{ Sem correção de franjas } & \multicolumn{3}{|c|}{ Diferença } \\
\hline Identificação & Nome & {$[\mathrm{CaH} 2]+[\mathrm{CaH} 3]$} & [TiO5] & $\zeta_{\mathrm{TiO} / \mathrm{CaH}}(\mathrm{L} 12)$ & {$[\mathrm{CaH} 2]+[\mathrm{CaH} 3]$} & [TiO5] & $\zeta_{\mathrm{TiO} / \mathrm{CaH}}(\mathrm{L} 12)$ & {$[\mathrm{CaH} 2]+[\mathrm{CaH} 3]$} & {$[\mathrm{TiO} 5]$} & $\zeta_{\mathrm{TiO} / \mathrm{CaH}}$ \\
\hline 2 & $\mathrm{PM}_{-}-\mathrm{I} 01008-2126$ & 1.39 & 0.65 & 1.44 & 1.39 & 0.64 & 1.07 & 0.00 & 0.00 & 0.37 \\
\hline 3 & $\mathrm{PM}_{-}-\mathrm{I} 00426-2314$ & 1.53 & 0.66 & 1.03 & 1.46 & 0.63 & 1.34 & 0.07 & 0.03 & -0.31 \\
\hline 4 & $\mathrm{PM}_{--\mathrm{I} 01266-4842 \mathrm{E}}$ & 1.10 & 0.36 & 1.09 & 1.13 & 0.37 & 1.14 & -0.03 & 0.00 & -0.05 \\
\hline 5 & $\mathrm{PM}_{-}-\mathrm{I} 01543-2249 \mathrm{~N}$ & 1.16 & 0.42 & 1.09 & 1.18 & 0.41 & 1.13 & -0.02 & 0.00 & -0.04 \\
\hline 6 & $\mathrm{PM}_{-}-\mathrm{I} 02300-2402 \mathrm{E}$ & 1.53 & 0.74 & 1.11 & 1.58 & 0.75 & 1.34 & -0.05 & -0.01 & -0.23 \\
\hline 7 & $\mathrm{PM}_{-}-\mathrm{I} 02462-2311$ & 1.79 & 0.83 & 2.05 & 1.73 & 0.79 & 2.01 & 0.07 & 0.04 & 0.04 \\
\hline 8 & $\mathrm{PM}_{-}-\mathrm{I} 02542-6619$ & 1.25 & 0.48 & 1.15 & 1.30 & 0.48 & 1.25 & -0.04 & -0.01 & -0.11 \\
\hline 9 & PM_-I04325-5657S & 1.43 & 0.69 & 0.87 & 1.45 & 0.64 & 1.25 & -0.01 & 0.05 & -0.37 \\
\hline 10 & $\mathrm{PM}_{-}-\mathrm{I} 04477-3044 \mathrm{E}$ & 1.06 & 0.51 & 0.78 & 1.08 & 0.52 & 0.81 & -0.02 & 0.00 & -0.03 \\
\hline 12 & $\mathrm{PM}_{-}-\mathrm{I} 05425-4305 \mathrm{~W}$ & 1.17 & 0.40 & 1.14 & 1.14 & 0.38 & 1.14 & 0.03 & 0.02 & 0.00 \\
\hline 13 & $\mathrm{PM}_{-}-\mathrm{I} 05442-2417 \mathrm{~N}$ & 1.18 & 0.48 & 0.99 & 1.21 & 0.51 & 1.02 & -0.04 & -0.03 & -0.02 \\
\hline 14 & $\mathrm{PM}_{-}-\mathrm{I} 05560-4511$ & 1.09 & 0.38 & 1.04 & 1.10 & 0.38 & 1.06 & -0.01 & -0.01 & -0.02 \\
\hline 15 & PM_-I06276-2356 & 1.39 & 0.63 & 1.07 & 1.40 & 0.62 & 1.17 & -0.01 & 0.01 & -0.09 \\
\hline 16 & $\mathrm{PM}_{--\mathrm{I}} 08239-7549 \mathrm{E}$ & 0.99 & 0.35 & 0.98 & 1.00 & 0.34 & 0.99 & -0.01 & 0.00 & -0.01 \\
\hline 17 & $\mathrm{PM}_{-}-\mathrm{I} 08386-3857$ & 1.23 & 0.49 & 1.07 & 1.24 & 0.49 & 1.10 & 0.00 & 0.00 & -0.03 \\
\hline 18 & PM_-I13133-4153S & 1.19 & 0.60 & 0.77 & 1.17 & 0.58 & 0.80 & 0.03 & 0.02 & -0.03 \\
\hline 19 & $\mathrm{PM}_{--\mathrm{I} 18241-5107}$ & 1.09 & 0.40 & 1.01 & 1.10 & 0.40 & 1.03 & -0.01 & 0.00 & -0.03 \\
\hline 21 & $\mathrm{PM}_{-}-\mathrm{I} 20371-5816$ & 1.38 & 0.57 & 1.22 & 1.37 & 0.55 & 1.27 & 0.02 & 0.01 & -0.05 \\
\hline 22 & $\mathrm{PM}_{-}-\mathrm{I} 21175-4142 \mathrm{~W}$ & 1.36 & 0.57 & 1.15 & 1.36 & 0.58 & 1.18 & 0.00 & 0.00 & -0.02 \\
\hline 25 & $\mathrm{PM}_{-}-\mathrm{I} 22487-5613 \mathrm{E}$ & 1.28 & 0.53 & 1.07 & 1.29 & 0.54 & 1.10 & -0.01 & -0.01 & -0.04 \\
\hline 26 & $\mathrm{PM}_{--} \mathrm{I} 23034-5311$ & 1.39 & 0.70 & 0.88 & 1.38 & 0.70 & 0.89 & 0.00 & 0.00 & -0.01 \\
\hline 27 & $\mathrm{PM}_{-}-\mathrm{I} 23099-2056$ & 1.64 & 0.83 & 1.03 & 1.58 & 0.80 & 1.04 & 0.06 & 0.03 & -0.01 \\
\hline
\end{tabular}




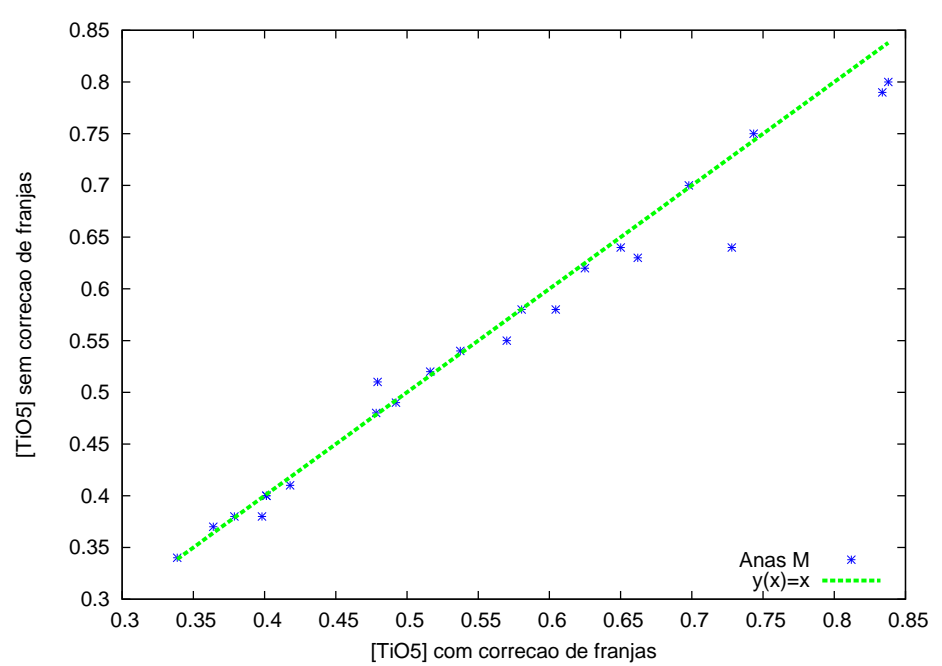

(a)

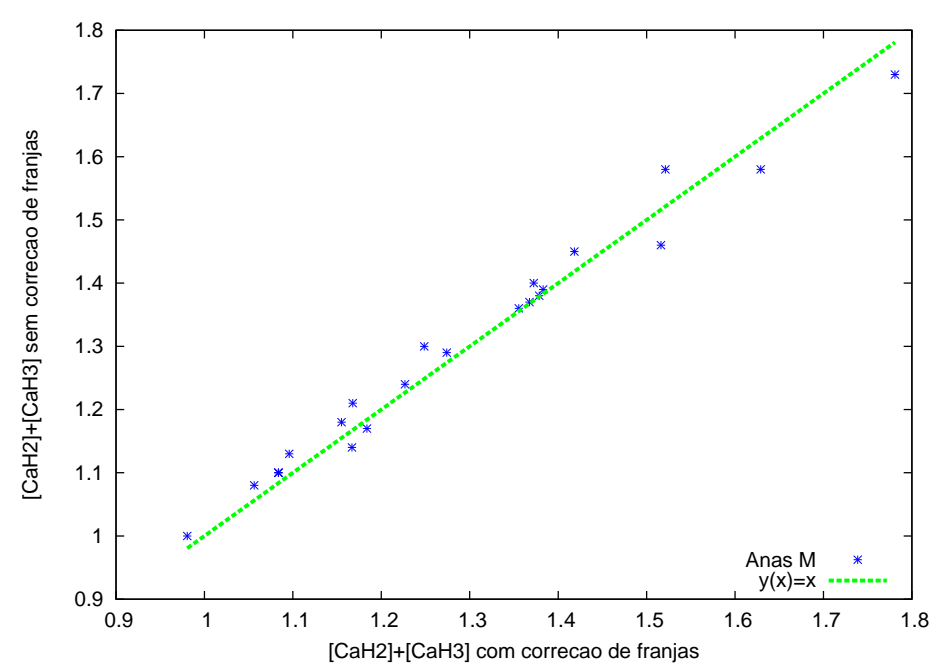

(b)

Figura 4.5: Efeito da correção de franjas: (a) Comparação entre o índice de TiO5 calculado com e sem correção de franjas. (b) Comparação entre a soma dos índices de CaH2+CaH3 calculado com e sem correção de franjas.

\subsection{Os sistemas triplos}

Como mostrado nas tabelas (2.1) e (2.2), a amostra conta com dois sistemas triplos. O par 6 é constituído de uma estrela de tipo espectral F ou G e duas de tipo M. Este sistema poderia ser usado para testar a consistência do índice $\zeta_{\mathrm{TiO} / \mathrm{CaH}}$ entre as secundárias, mas uma das estrelas M apresentou razão $\mathrm{S} / \mathrm{N}<20$ e não pôde ser utilizada na análise. Assim, este sistema será tratado como um sistema duplo apenas para fins de calibração de metalicidade.

O par 19, por sua vez, é constítuido de duas estrelas de tipo espectral F ou G e uma estrela de tipo M. Esperava-se que as estrelas F/G apresentassem a mesma metalicidade, assumindo a origem comum de ambas, mas não foi isto que ocorreu. A estrela PM_18240$5107 \mathrm{~N}$ apresentou $[\mathrm{Fe} / \mathrm{H}]=-0.54$ e a estrela $\mathrm{PM}_{-} 18240-5107 \mathrm{~S}[\mathrm{Fe} / \mathrm{H}]=-0.33$ (Tabela 4.1), mas, essa diferença poderia ser compensada pelo erro típico de 0.2dex. Também era esperado que essas estrelas compartilhassem a mesma velocidade radial, já que se trata de um sistema extenso. Mas, quando compara-se as velocidades radias fornecidas pelo nsspp de $134.6 \pm 34.6 \mathrm{~km} / \mathrm{s}$ para $\mathrm{PM}_{-} 18240-5107 \mathrm{~N}$ e $76.5 \pm 8.4 \mathrm{~km} / \mathrm{s}$ para $\mathrm{PM}_{-} 18240-5107 \mathrm{~S}$, nota-se que elas diferem em um valor que ultrapassa o erro. Por este sistema levantar dúvidas quanto a sua natureza múltipla e origens, a estrela M, PM_18241-5107 sempre 
será apresentada distintamente nos gráficos de abundâncias.

\subsection{Subtipos espectrais}

Gizis et al. (1997) calibraram relações baseadas nas bandas de CaH2, CaH3, TiO5, VO1, TiO6, VO2 e TiO7 para determinação de subtipos espectrais (Sp) de estrelas M entre M0 e M6. Apesar das relações serem válidas apenas neste intervalo, $\mathrm{Sp} \approx-1$ indica uma estrela $\mathrm{K} 7$ e $\mathrm{Sp} \approx-2$ indica uma K5. A fim de determinar os subtipos espectrais da amostra aqui tratada, utilizou-se as calibrações de G97 com as bandas de CaH2, CaH3 e TiO5:

$$
\begin{aligned}
& S p=7.91[\mathrm{CaH} 2]^{2}-20.63[\mathrm{CaH} 2]+10.71 \\
& S p=-18[\mathrm{CaH} 3]+15.80 \\
& S p=-9.64[\mathrm{TiO} 5]+7.76 .
\end{aligned}
$$

Os subtipos espectrais são determinados arredondando-se os resultados das equações (4.1, 4.2 e 4.3) pelo seu inteiro ou semi-inteiro mais próximo. A Tabela (4.4) apresenta os subtipos espectrais determinados para a amostra. A dispersão média nas determinações utilizando-se as diferentes bandas foi de 0.6 classes espectrais. É importante notar que nenhuma das estrelas foi classificada com subtipo $\mathrm{M} \geq 5$, ou seja tem-se, neste trabalho, uma amostra somente de estrelas M tipo jovem.

Ainda em relação à amostra deste trabalho, também se pode notar mudanças nas profundidades das bandas de CaH e TiO5 em função da variação dos subtipos espectrais, mostradas na Figura (1.2). A Figura (4.6) mostra como a força das bandas de CaH2 e TiO5 vão se igualando conforme caminha-se para subtipos mais tardios, conforme o esperado. 


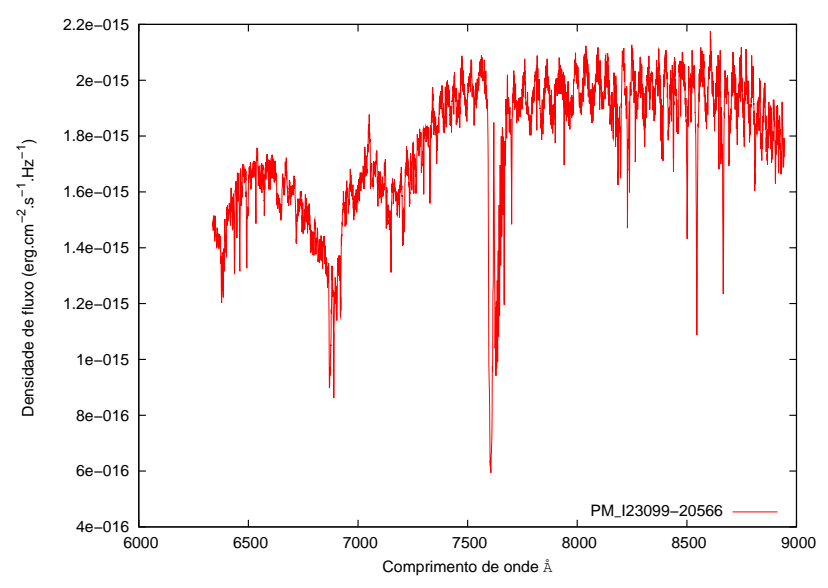

(a) M0

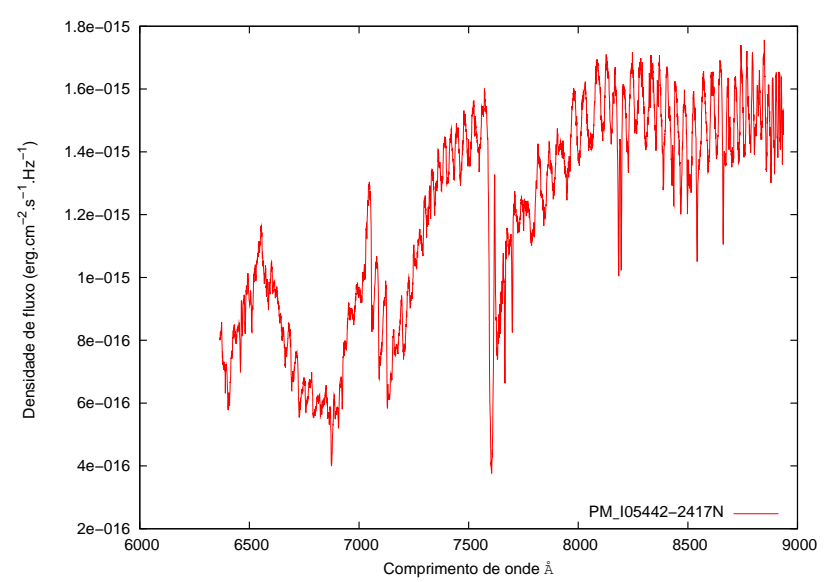

(c) M3.0

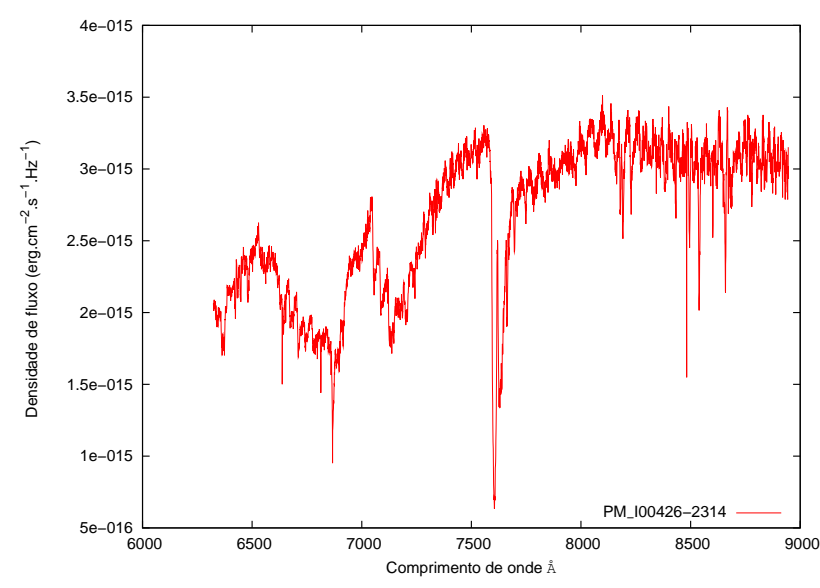

(b) M1.5

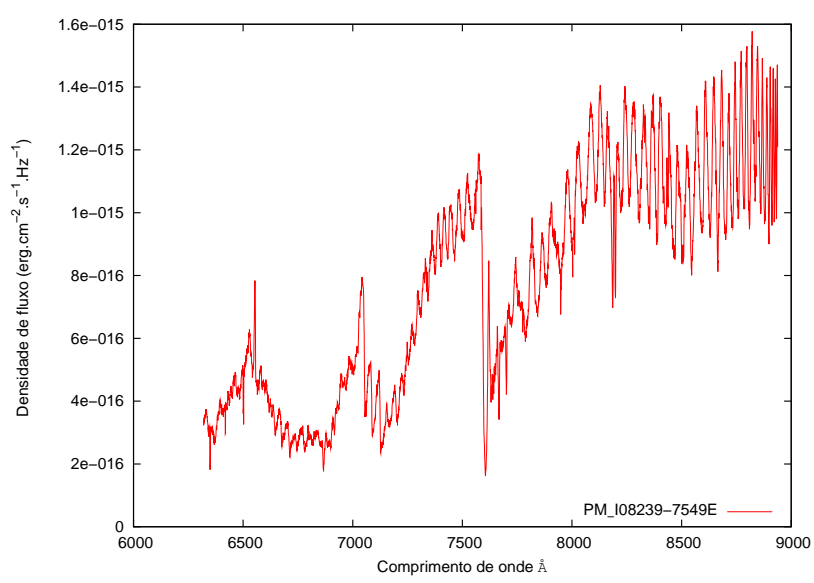

(d) M4.5

Figura 4.6: Variação nas bandas de $\mathrm{CaH}$ e TiO5 com a mudança dos subtipos espectrais 
Tabela 4.4 - Subtipos espectrais das estrelas secundárias da amostra desse trabalho de acordo com as calibrações de G97. As determinações foram feitas utilizando as bandas de $\mathrm{CaH} 2$, CaH3 e TiO5.

\begin{tabular}{|c|l|c|c|c|c|}
\hline \multicolumn{2}{|c|}{ Estrelas } & \multicolumn{2}{c|}{ Subtipos espectrais } & \multirow{2}{*}{ Desvio } \\
\cline { 1 - 4 } Identificação & Nome & CaH2 & CaH3 & TiO5 & padrão \\
\cline { 1 - 3 } 3 & PM_I01008-2126 & M0.5 & M0.5 & M1.5 & 0.6 \\
4 & PM_I00426-2314 & M1.0 & M1.5 & M1.5 & 0.3 \\
5 & PM_I01266-4842E & M3.5 & M3.5 & M4.5 & 0.6 \\
6 & PM_I01543-2249N & M3.0 & M3.0 & M3.5 & 0.3 \\
7 & PM_I02300-2402E & M0.5 & M0.5 & M0.5 & 0.0 \\
8 & PM_I02462-2311 & K7 & K7 & M0 & 0.6 \\
9 & PM_I02542-6619 & M2.5 & M2.0 & M3.0 & 0.5 \\
10 & PM_I04325-5657S & M1.0 & M1.0 & M0.5 & 0.3 \\
12 & PM_I04477-3044E & M3.5 & M4.5 & M3.0 & 0.8 \\
13 & PM_I05425-4305W & M3.0 & M2.5 & M4.0 & 0.8 \\
14 & PM_I05442-2417N & M3.0 & M3.0 & M3.0 & 0.0 \\
15 & PM_I05560-4511 & M3.5 & M3.5 & M4.0 & 0.3 \\
16 & PM_I06276-2356 & M1.0 & M2.0 & M1.5 & 0.5 \\
17 & PM_I08239-7549E & M4.0 & M4.5 & M4.5 & 0.3 \\
18 & PM_I08386-3857 & M2.5 & M2.5 & M3.0 & 0.3 \\
19 & PM_I13133-4153S & M2.5 & M3.0 & M2.0 & 0.5 \\
21 & PM_I18241-5107 & M3.5 & M4.0 & M4.0 & 0.3 \\
22 & PM_I20371-5816 & M1.5 & M1.5 & M2.5 & 0.6 \\
25 & PM_I21175-4142W & M1.5 & M1.5 & M2.0 & 0.3 \\
26 & PM_I22487-5613E & M2.0 & M2.5 & M2.5 & 0.3 \\
27 & PM_I23099-2056 & M0 & M0 & M0 & 0.0 \\
\hline
\end{tabular}

\subsection{Comparação com outros trabalhos}

\subsubsection{Diagrama $[\mathrm{CaH} 2]+[\mathrm{CaH} 3]$ versus $[\mathrm{TiO} 5]$}

Como primeira análise, construiu-se diagramas de [CaH2]+[CaH3] versus [TiO5] para fins de comparação com o obtido por Woolf et al. (2009) (Figura 3.2). O resultado está apresentado na Figura (4.7). Nota-se que o diagrama segue a mesma distribuição e escalas da Figura (3.2). Entretanto, não se percebe a mesma separação de metalicidades que a obtida por eles. Tal separação somente pode ser notada a partir de uma amostra mais numerosa de estrelas, como a utilizada por Woolf et al. (2009). Assim, o número reduzido de objetos neste trabalho pode ter impossibilitado a identificação da separação. 

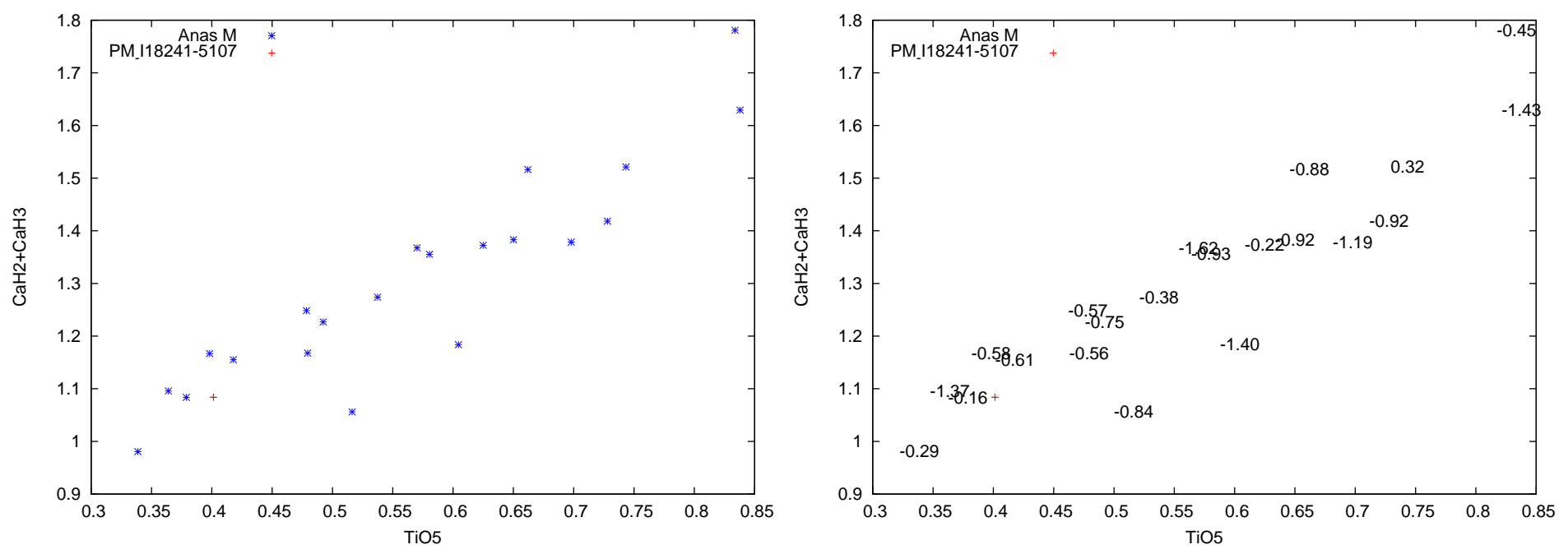

Figura 4.7: Diagrama [CaH2] +[CaH3] versus [TiO5] mostrando a localização da nossa amostra de anãs M (figura da esquerda) e as respectivas metalicidades delas (figura da direita).

\subsection{2 $K_{\text {index }}$ e a metalicidade}

Nos catálogos LSPM-north e LSPM-south, os autores apresentam um índice cinemático chamado $\mathrm{K}_{\text {index }}$ que está associado à metalicidade e, consequentemente, com a localização do par no halo galáctico. Os critérios de metalicidade para o $\mathrm{K}_{\text {index }}$ foram apresentados na seção (2.1). Nesta seção, tais critérios foram testados utilizando as metalicidades estimadas via n-sspp para os sistemas. A Figura (4.8) mostra que se existe uma relação entre a metalicidade e $\mathrm{K}_{\text {index }}$, ela é mínima. Esta verificação não necessita de nenhum dado obtido dos espectros das estrelas secundárias, de modo que a razão $\mathrm{S} / \mathrm{N}$ dos objetos não interfere na análise. Isso possibilitou construir um gráfico para todos os pares observados (Figura 4.8(b)) aumentando a amostra numérica e, consequentemente, a confiança nos resultados. Note-se a fraca correlação entre $\mathrm{K}_{\text {index }}$ e a metalicidade. Os dados de metalicidade para toda a amostra podem ser encontrados na Tabela (A.1). 


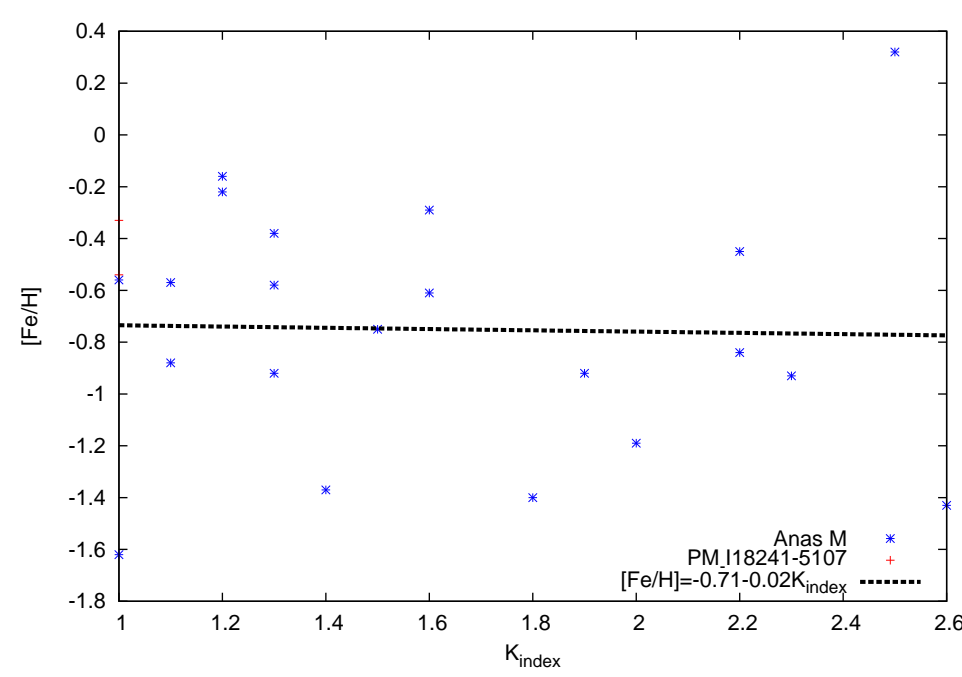

(a)

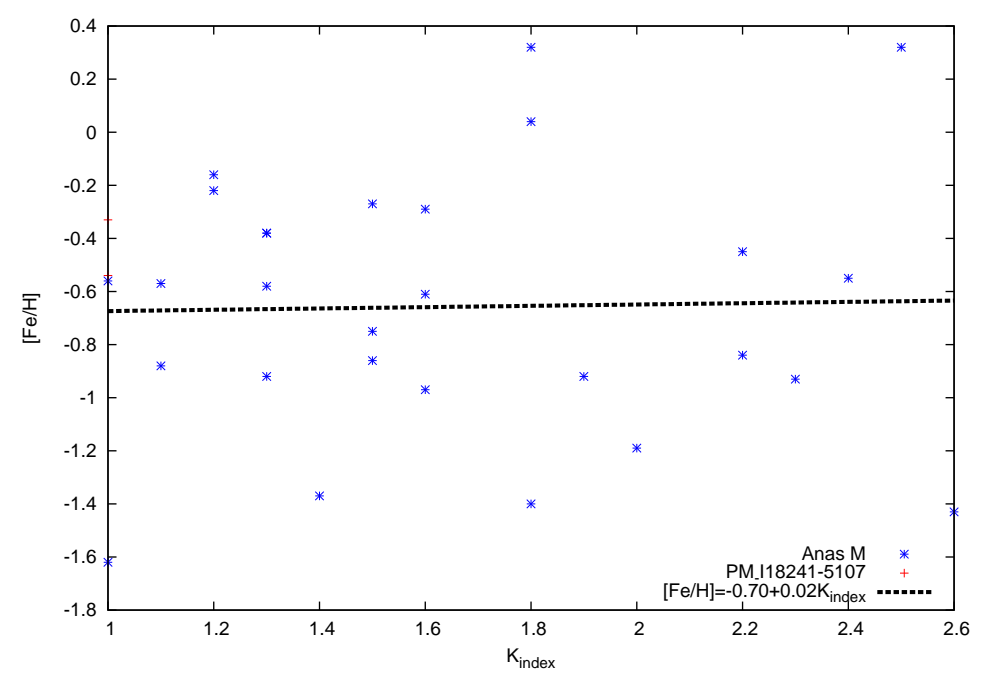

(b)

Figura 4.8: $[\mathrm{Fe} / \mathrm{H}]$ versus $\mathrm{K}_{\text {index }}$ : (a) Amostra de sistemas com anãs $\mathrm{M}$ com $\mathrm{S} / \mathrm{N}>20$. (b) Amostra com todos os sistemas observados.

\subsection{3 $\zeta_{\mathrm{TiO} / \mathrm{CaH}} \mathrm{como}$ indicador de metalicidade}

A fim de calibrar uma relação de metalicidade para anãs $\mathrm{M}$ em função de $\zeta_{T i O / C a H}$, assim como fez Woolf et al. (2009) (Figura 3.5), construiu-se gráficos de $[\mathrm{Fe} / \mathrm{H}]$ versus $\zeta_{\mathrm{TiO} / \mathrm{CaH}}$ utilizando as calibrações para $[\mathrm{TiO} 5]_{Z_{\odot}}$ de Lépine et al. $(2007,2012)$ e Dithal et al. (2012).

Como discutido anteriormente, a diferença entre as distintas calibrações de $[\mathrm{TiO} 5]_{Z_{\odot}}$ aparece predominantemente em estrelas $\mathrm{M}$ tipo jovem. Como a amostra deste trabalho é constituída por objetos desses tipo espectrais, pode-se prever que os gráficos utilizando tais calibrações serão nitidamente diferentes entre si. Os resultados estão apresentados nas figuras (4.9), (4.10) e (4.11).

Não é possível notar qualquer correlação entre $\zeta_{T i O / C a H}$ e $[\mathrm{Fe} / \mathrm{H}]$ nas figuras (4.9) e (4.11), mas uma fraca correlação pode ser percebida na Figura (4.10). Um ajuste foi obtido para a calibração de Dithal et al. (2012) utilizando toda amostra válida (curva rosa):

$$
[\mathrm{Fe} / \mathrm{H}]=(-1.6 \pm 0.7)+(0.88 \pm 0.78) \zeta_{\mathrm{TiO} / \mathrm{CaH}}
$$

Obteve-se também, um ajuste excluindo a estrela PM_02300-2402E (par 6) pois sua metalicidade estimada $([\mathrm{Fe} / \mathrm{H}] \sim 0.4)$ a situa fora do conjunto de estrelas pobres em metais. $\mathrm{O}$ 
ajuste (curva azul) nesta condição foi:

$$
[F e / H]=(-1.9 \pm 0.7)+(1.2 \pm 0.7) \zeta_{T i O / C a H}
$$

Com esta última calibração, os ajustes deste trabalho e de Woolf et al. (2009) se tornaram mais paralelos entre si quando comparado com a calibração da equação (4.4). Na Figura (4.12) mostra-se a reta obtida via equações (4.4) e (4.5), bem como o ajuste obtido por Woolf et al. (2009), para fins de comparação.

Note-se que a estrela do par número 7, classificada como K7, apresentou $\zeta_{\mathrm{TiO} / \mathrm{CaH}}$ fora do intervalo típico para este índice (Tabela 4.1) quando utilizou-se as equações de Lépine et al. (2007) e Lépine et al. (2012). Logo, a referida estrela foi retirada das Figuras (4.9) e (4.11).

Apesar do trabalho de Woolf et al. (2009) ter resultado em uma boa calibração de $[\mathrm{Fe} / \mathrm{H}]$ em função de $\zeta_{T i O / C a H}$, o trabalho de Lépine et al. (2012), realizado em baixa/média resolução, mostrou uma baixa correlação entre $\zeta_{T i O / C a H}$ e $[\mathrm{Fe} / \mathrm{H}]$, onde a metalicidade foi obtida através de outras calibrações como a de Neves et al. (2012) e Rojas-Ayala et al. (2010). A Figura (4.13) mostra os resultados.

Lépine et al. (2012) também mostraram que os índices espectrais [CaH2], [CaH3] e [TiO5] são dependentes da instrumentação utilizada durante as observações. Eles observaram uma amostra de 484 estrelas nos observatórios MDM e UH que já havia sido observada por Reid et al. (1995) no observatório Palomar-MSU e compararam os resultados para os diferentes observatórios entre si (Figura 4.14). Pode-se notar que a maior parte das diferenças são sistemáticas e podem ser corrigidas, mas uma parte delas é aleatória sem possibilidade de correção. Apesar dessas diferenças serem pequenas, fica claro que esses índices são bastante afetados pela instrumentação utilizada.

Outro teste importante feito por Lépine et al. (2012) foi a análise da precisão na determinação do $\zeta_{T i O / C a H}$ em função do subtipo espectral da estrela. O teste resultou na diminuição da precisão de $\zeta_{T i O / C a H}$ para subtipos espectrais mais iniciais, como pode ser observado na Figura (4.15). O espalhamento em $\zeta_{\mathrm{TiO} / \mathrm{CaH}}$ é bastante grande para subti- 
pos inferiores a M2, principalmente quando se compara com as diferenças em $\zeta_{\mathrm{TiO} / \mathrm{CaH}}$ das tabelas (4.2) e (4.3), que dizem respeito ao efeito da correção de franjas e calibração em fluxo sobre o índice. Considerando ainda que, o intervalo típico de $\zeta_{\mathrm{TiO} / \mathrm{CaH}}$ vai apenas de aproximadamente 0.2 a 1.2 , dispersões de 0.5 e 0.2 como as mostradas na Figura (4.15) representam incertezas de $36 \%$ e 14\%, respectivamente. Assim, uma parcela da má calibração entre $[\mathrm{Fe} / \mathrm{H}]$ e $\zeta_{T i O / C a H}$ que se obteve neste trabalho pode ser atribuida à imprecisão na determinação de $\zeta_{T i O / C a H}$ para subtipos mais jovens, à dependência de $\zeta_{T i O / C a H}$ com a instrumentação e aos erros em $[\mathrm{Fe} / \mathrm{H}]$ obtidos pelo estimador n-sspp.

Finalmente, pode-se concluir que, a utilização do índice $\zeta_{\mathrm{TiO} / \mathrm{CaH}}$ como indicador de metalicidade deve ser feita com bastante cautela, dada sua sensibilidade a diferentes fatores. Pode-se mesmo questionar a validade da relação entre o índice e a metalicidade. 


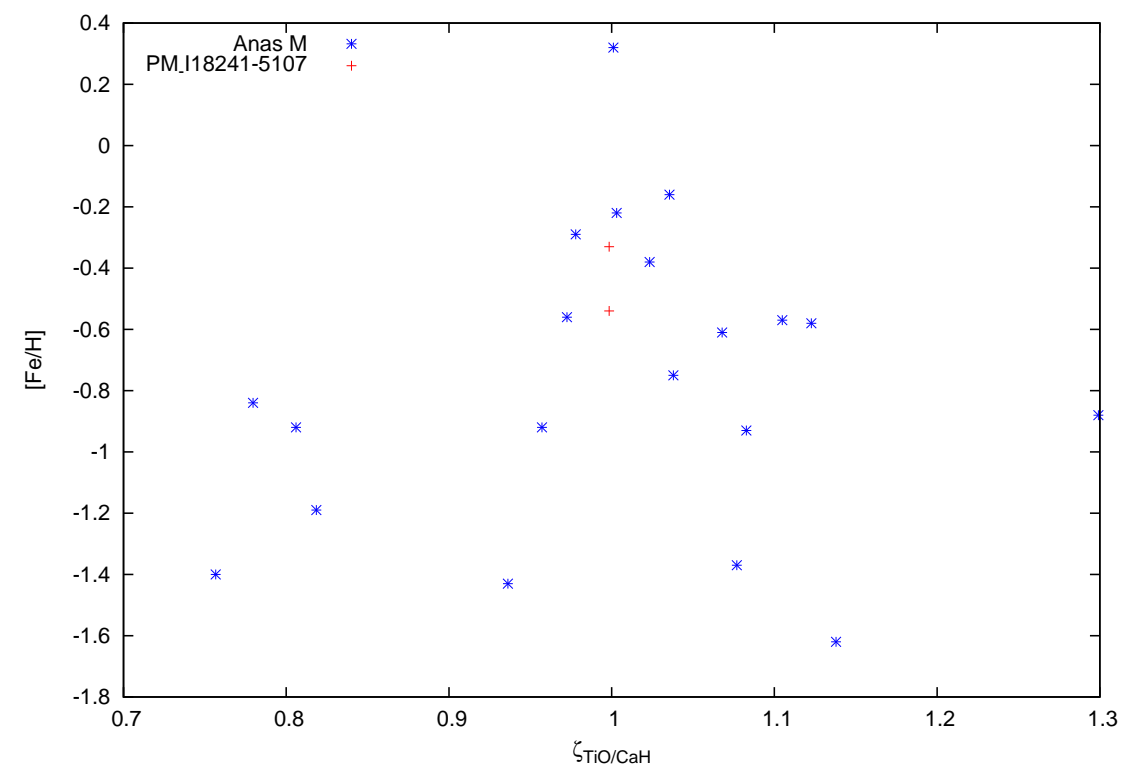

Figura 4.9: $[\mathrm{Fe} / \mathrm{H}]$ versus $\zeta_{\mathrm{TiO} / \mathrm{CaH}}$ utilizando a equação para $[\mathrm{TiO} 5]_{Z_{\odot}}$ de Lépine et al. $(2007)$

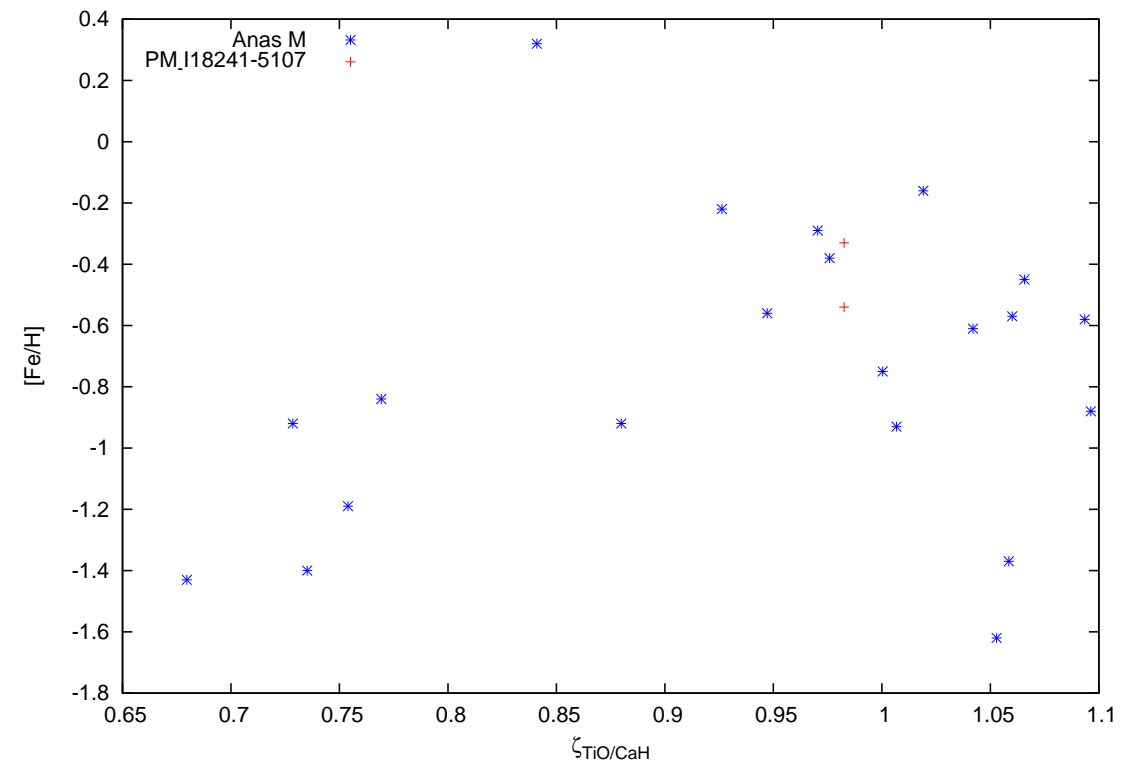

Figura 4.10: $[\mathrm{Fe} / \mathrm{H}]$ versus $\zeta_{\mathrm{TiO} / \mathrm{CaH}}$ utilizando a equação para $[\mathrm{TiO} 5]_{Z_{\odot}}$ de Dithal et al. (2012) 


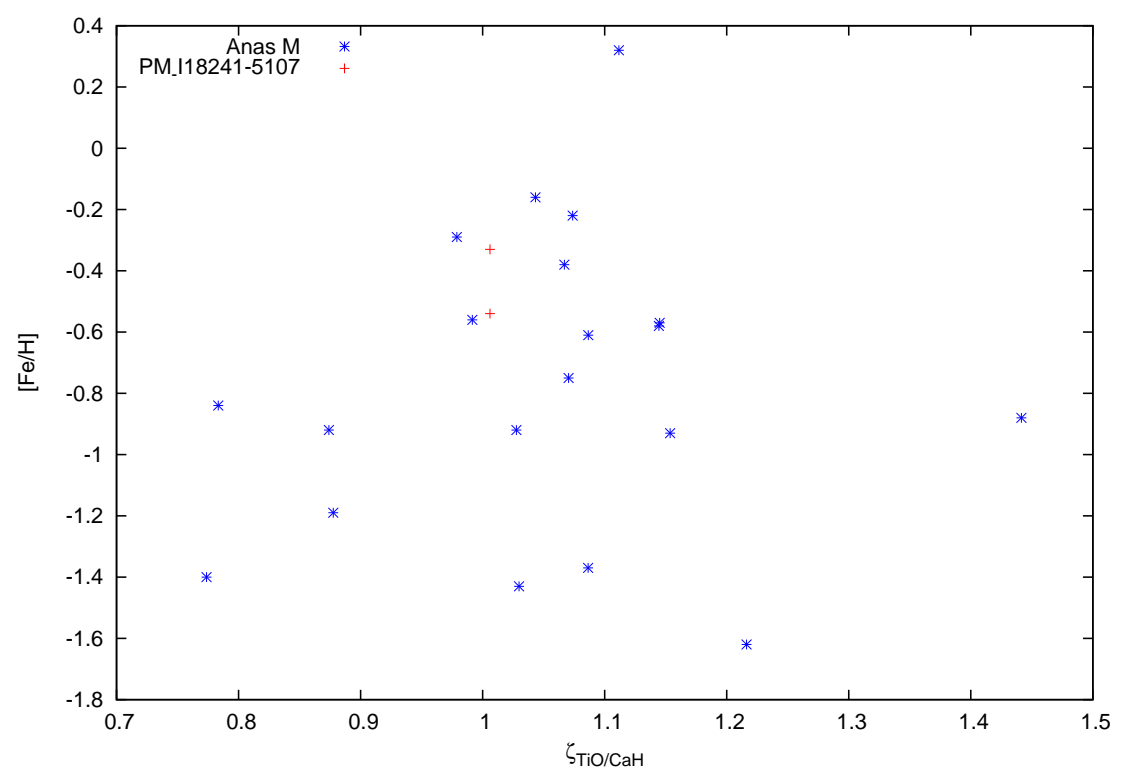

Figura 4.11: $[\mathrm{Fe} / \mathrm{H}]$ versus $\zeta_{T i O / C a H}$ utilizando a equação para $[\mathrm{TiO} 5]_{Z_{\odot}}$ de Lépine et al. (2012)

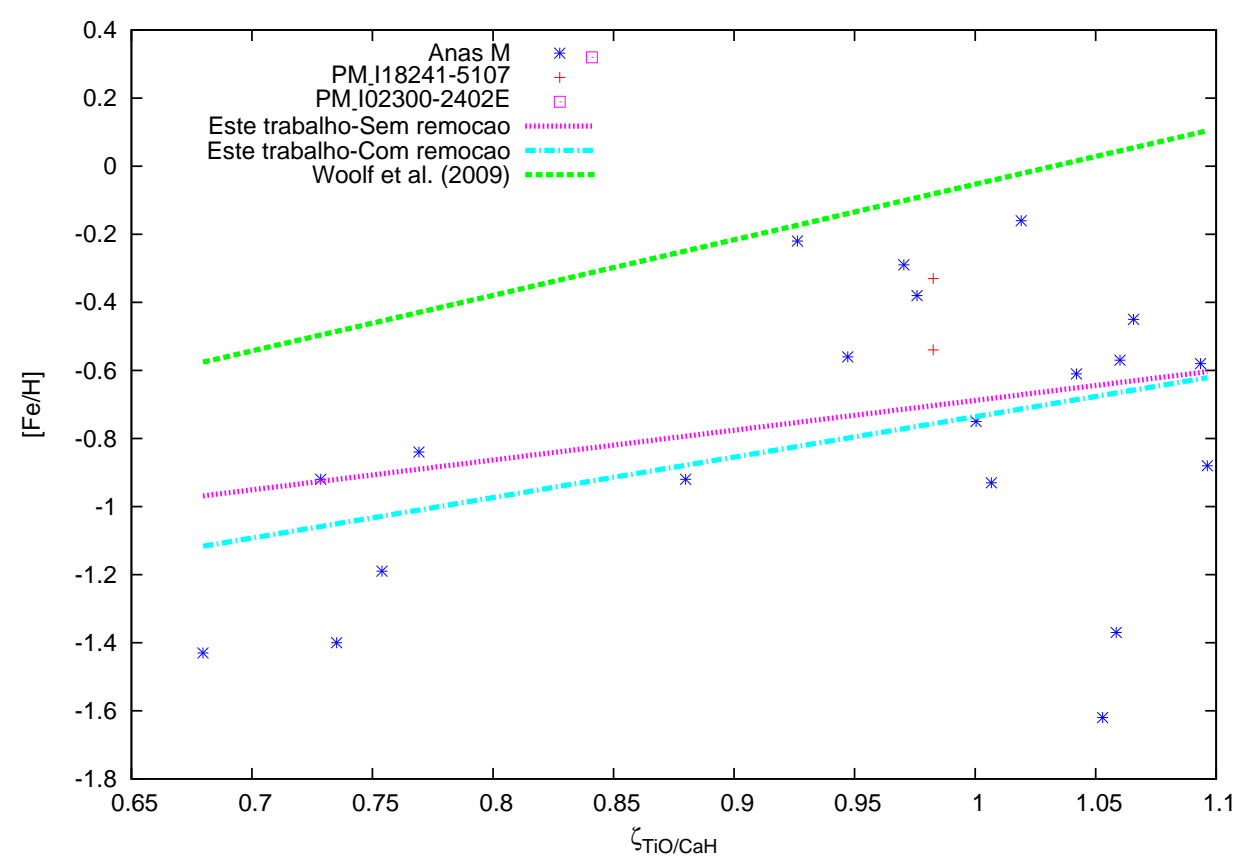

Figura 4.12: $[\mathrm{Fe} / \mathrm{H}]$ versus $\zeta_{T i O / C a H}$ utilizando a equação para $[\mathrm{TiO} 5]_{Z_{\odot}}$ de Dithal et al. (2012). A reta em rosa mostra o ajuste para toda amostra válida: $[\mathrm{Fe} / \mathrm{H}]=(-1.6 \pm$ $0.7)+(0.88 \pm 0.78) \zeta_{\mathrm{TiO} / \mathrm{CaH}}$. A reta em azul mostra o ajuste excluindo a estrela PM_02300-2402E: $[\mathrm{Fe} / \mathrm{H}]=(-1.9 \pm 0.7)+(1.2 \pm 0.7) \zeta_{\text {TiO/CaH}}$. A reta em verde mostra a calibração de Woolf et al. (2009). 

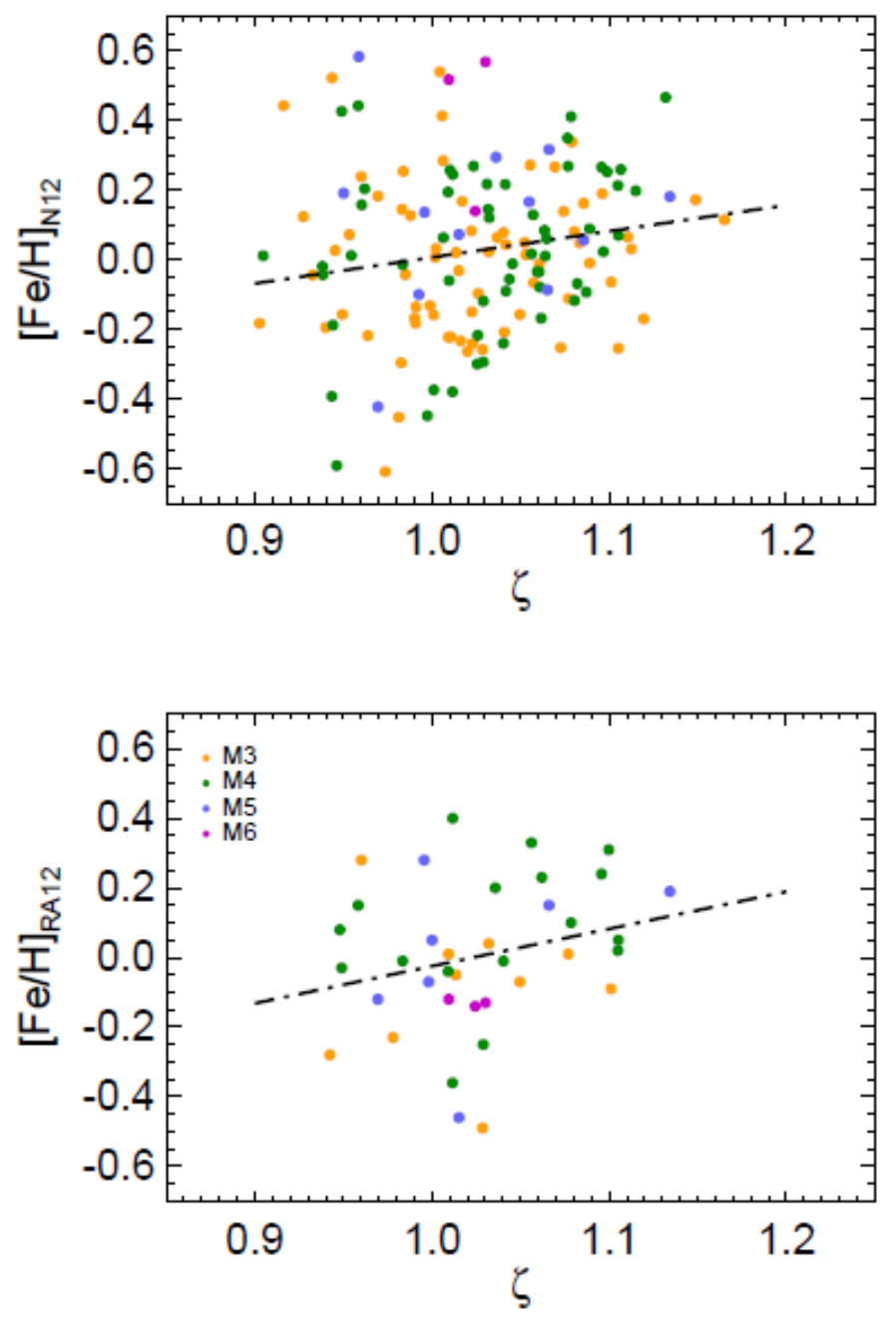

Figura 4.13: Comparação entre $\zeta_{\mathrm{TiO} / \mathrm{CaH}}$ e metalicidades obtidas por métodos independentes para uma amostra de anãs $\mathrm{M}$ feita por Lépine et al. (2012). O painel de cima compara $\zeta_{\text {TiO/CaH }} \operatorname{com}[\mathrm{Fe} / \mathrm{H}]$ obtido através da calibração fotométrica de Neves et al. (2012). O painel de baixo compara $\zeta_{\mathrm{TiO} / \mathrm{CaH}} \mathrm{com}[\mathrm{Fe} / \mathrm{H}]$ obtido pela calibração no infravermelho de Rojas-Ayala (2010). 

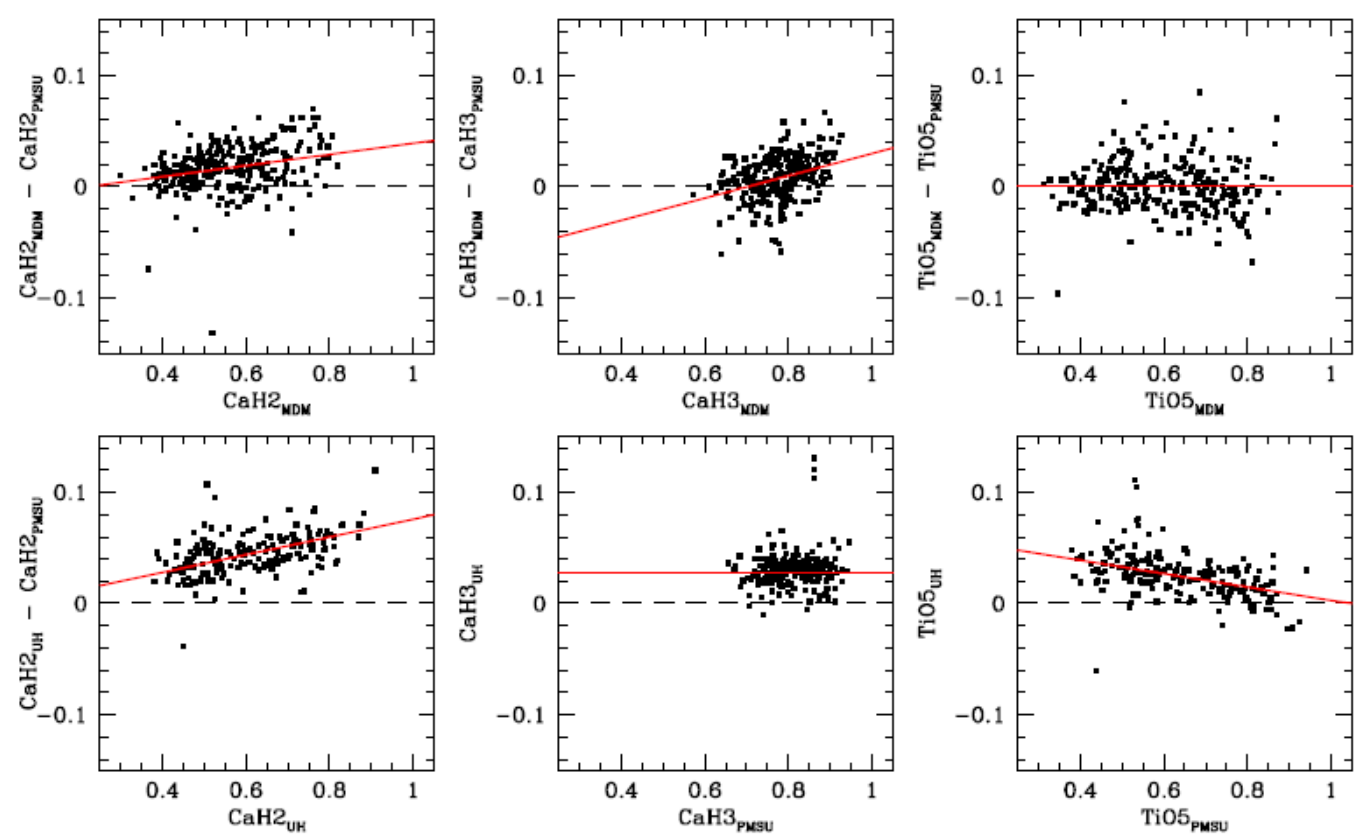

Figura 4.14: Comparação feita por Lépine et al. (2012) entre índices espectrais medidos a partir de três diferentes observatórios (MDM, Palomar-MSU e UH), mostrando a dependência instrumental desses índices.

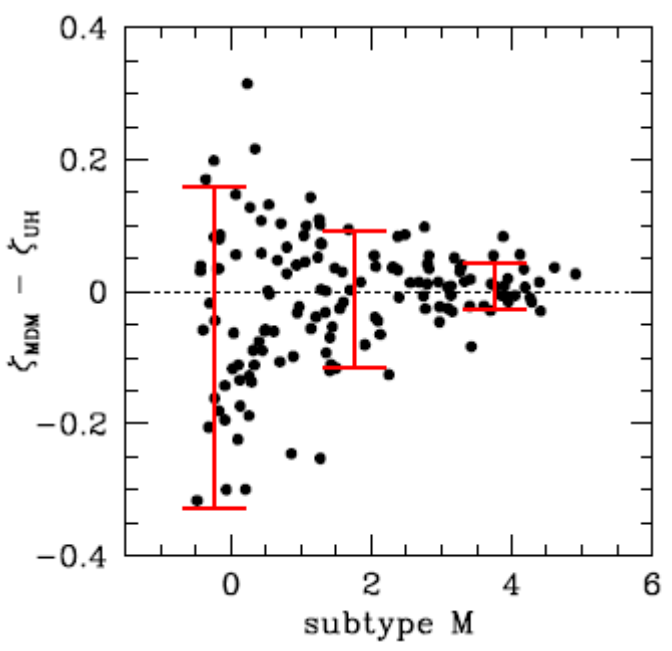

Figura 4.15: Teste feito por Lépine et al. (2012) do $\zeta_{T i O / C a H}$ em função do subtipo espectral mostrando que a imprecisão na medição de $\zeta_{\mathrm{TiO} / \mathrm{CaH}}$ aumenta para subtipos mais jovens. 


\subsection{Carbonicidade}

O n-sspp também forneceu estimativas de $[\mathrm{C} / \mathrm{Fe}]^{1}$ (chamado carbonicidade) para a amostra deste estudo (Tabela 4.1). Para análise, construíu-se, inicialmente, um gráfico de [C/Fe] versus $[\mathrm{Fe} / \mathrm{H}]$ (Figura 4.16) a fim de verificar o enriquecimeto em carbono $([\mathrm{C} / \mathrm{Fe}]>0.7$, Carollo et al. 2012) com a queda da metalicidade (Rossi et al. 2005). Em acordo com Carollo et al. (2012) pode-se notar o enriquecimento para metalicidades a partir de $[F e / H]<-1.0$.

Verificou-se, também, se $\zeta_{\mathrm{TiO} / \mathrm{CaH}}$ poderia se relacionar com a carbonicidade. Para isso, gráficos de $[\mathrm{C} / \mathrm{Fe}]$ versus $\zeta_{T i O / C a H}$ são mostrados na Figura (4.17). Note-se uma sutil tendência de $[\mathrm{C} / \mathrm{Fe}]$ aumentar com a queda de $\zeta_{T i O / C a H}$ na Figura $(4.17(\mathrm{a}))$, mas somente uma amostra maior poderia confirmar tal correlação. Nenhuma correlação pode ser percebida na Figura (4.17(b)).

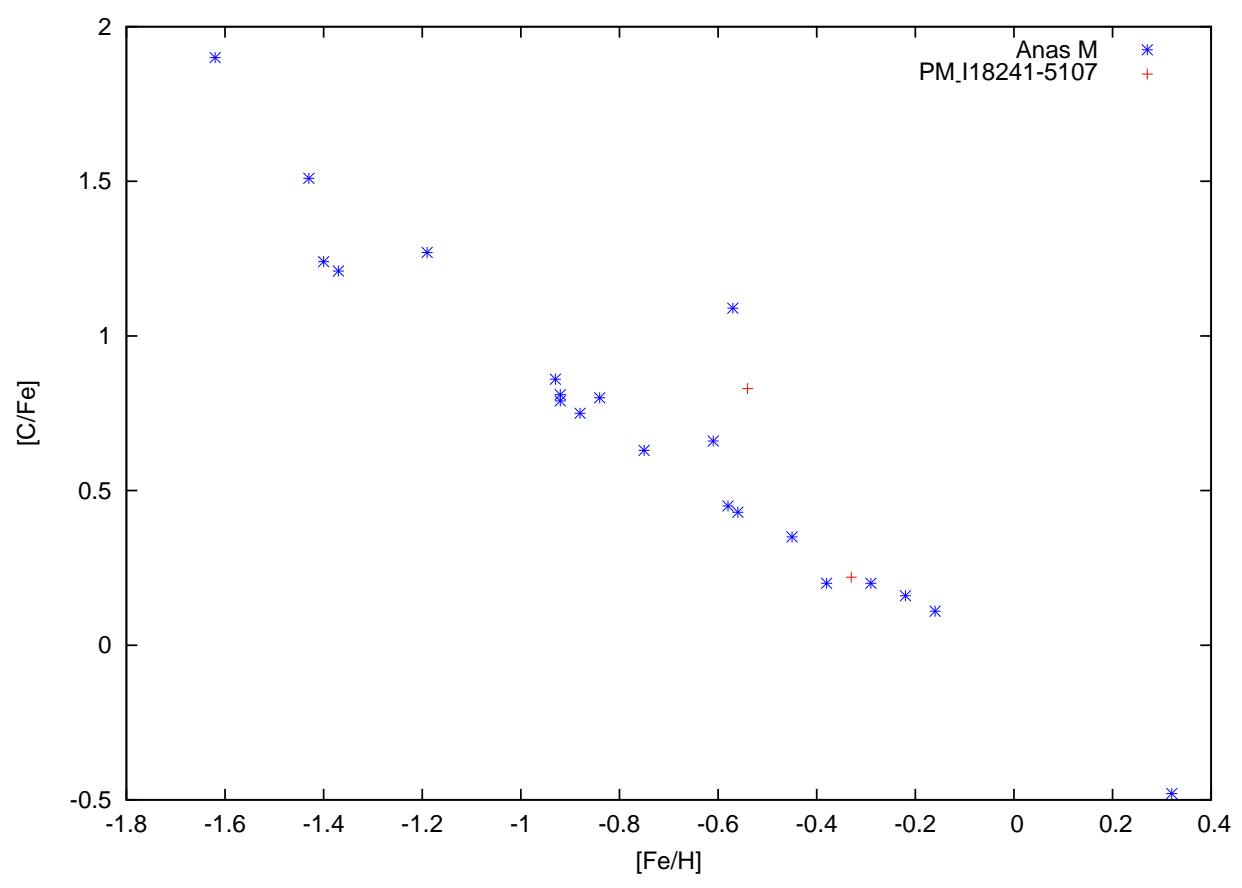

Figura 4.16: $[\mathrm{C} / \mathrm{Fe}]$ versus $[\mathrm{Fe} / \mathrm{H}]$ para a amostra de anãs M.

\footnotetext{
${ }^{1}[\mathrm{C} / \mathrm{Fe}]=\log \left(\frac{N_{C}}{N_{F e}}\right)_{*}-\log \left(\frac{N_{C}}{N_{F e}}\right)_{\odot}$, onde $N_{C}$ e $N_{F e}$ correspondem às abundâncias por número de átomos dos elementos $\mathrm{C}$ e Fe, respectivamente. Os índices $(*)$ e $(\odot)$ referem-se à estrela e ao Sol, respectivamente.
} 


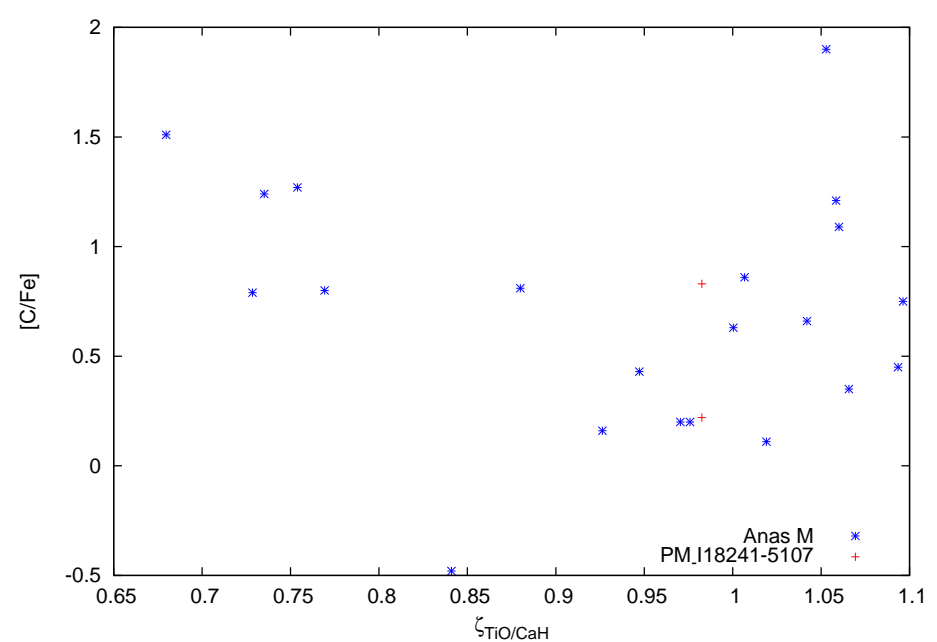

(a)

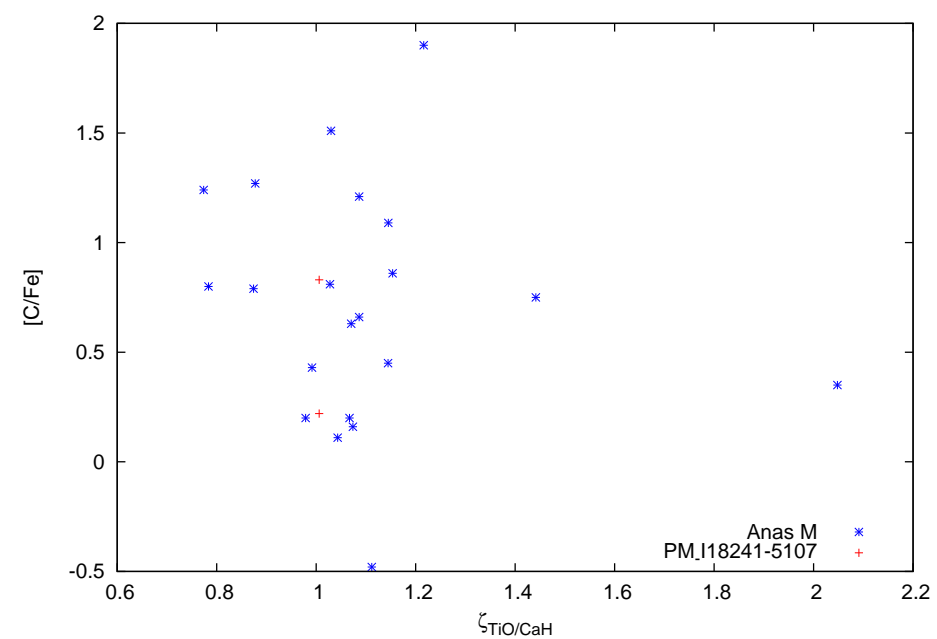

(b)

Figura 4.17: $[\mathrm{C} / \mathrm{Fe}]$ versus $\mathrm{K}_{\text {index }}$ : (a) $\zeta_{\mathrm{TiO} / \mathrm{CaH}} \mathrm{em}$ acordo com Dithal et al. (2012). $\zeta_{\mathrm{TiO} / \mathrm{CaH}}$ em acordo com Lépine et al. (2012). 
Capítulo 5

\section{Conclusões}

Este trabalho visou um estudo sobre calibrações de metalicidade de estrelas subanãs M. Primeiramente foi introduzido o contexto onde este trabalho está inserido. Em seguida, apresentou-se a metodologia utilizada, discutindo sobre o catálogo LSPM-south e a seleção de estrelas, sobre as configurações instrumentais do espectrógrafo Goodman e sobre a redução de dados. Apresentou-se também, a amostra de objetos observados neste trabalho (tabelas 2.1 e 2.2). Posteriormente, as calibrações de metalicidades para subanãs M existentes na literatura foram avaliadas para a amostra em estudo e apresentou-se aquela na qual este trabalho se focou.

Foram apresentados os resultados, começando pela validação no cálculo dos índices espectroscópicos. Mostrou-se também, que a calibração em fluxo pouco interfere nos valores desses índices. O procedimento de correção de franjas, também pouco afetou o resultado final dos índices, considerando que a instrumentação pode introduzir incertezas maiores nos índices do que aquelas causadas pelo efeito da calibração em fluxo e da correção de franjas. Após estimar os subtipos espectrais da amostra de anãs M utilizando bandas moleculares de $\mathrm{CaH}$ e TiO, encontrou-se que a mesma é constituída de anãs $\mathrm{M}$ tipo jovem.

Apresentou-se as tentativas de utilização do índice $\zeta_{\mathrm{TiO} / \mathrm{CaH}}$ como um indicador de metalicidade. Infelizmente não foi possível obter uma boa correlação entre $\zeta_{T i O / C a H}$ e $[\mathrm{Fe} / \mathrm{H}]$. Uma possível causa dessa falha de calibração pode ser o fato do índice $\zeta_{T i O / C a H}$ depender da instrumentação utilizada e dos subtipos espectrais analisados como foi averiguado no trabalho de Lépine et al. (2012). Este último, também encontrou uma débil correlação entre $\zeta_{T i O / C a H}$ e $[\mathrm{Fe} / \mathrm{H}]$ para uma amostra de anãs $\mathrm{M}$ do hemisfério norte. A partir da 
análise feita neste trabalho, concluí-se que $\zeta_{T i O / C a H}$ não é um bom calibrador de metalicidade, uma vez que se mostra carregado de várias incertezas. Por fim, apresentou-se os resultados de carbonicidade para amostra demostrando como estrelas pobres em metais tendem a ser enriquecidas em carbono. 


\section{Referências Bibliográficas}

Bahcall, J. N., \& Soneira, R. M. 1981, ApJ, 246, 122

Bean, J. L., Benedict, G. F., \& Endl, M. 2006, ApJ, 653, L65

Bessel, M. S. 1982, Proc. Astron. Soc. Australia, 4, 417

Bessel, M. S. 1991,AJ, 101, 662

Bidelman, W. P., \& Smethells, W. G. 1976, in IAU Symp. 72, Abundance Effects in Classification ed.

B. Hauck, P. C. Keenan \& W. W. Morgan (Boston: D. Reidel), p. 205

Bochanski, J. J., et al. 2010, AJ, 139, 2679

Boeshaar, P.C. 1976, Ph.D. Thesis, Ohio State Univ.

Boeshaar, P.C., \& Tyson, J.A, 1985, AJ, 90, 817

Bonfils, X., Delfosse, X., Udry, S., et al. 2005, A\&A, 442, 635

Burgasser, A., et al. 2003, ApJ, 592, 1186

Burgasser, A., \& Kirkpatrick, J. D. 2006, ApJ, 645, 1485

Burwell, C.G. 1930, PASP, 42, 351

Carollo, D. et al. 2012, ApJ, 744:195

Chanamé, J., \& Gould, A. 2004, ApJ, 601, 289

Chiappini, C. 2004, Sky and Telescope, 108, 32

Delfosse, X., Forveille, T., Segrasan, D., et al. 2000, A \& A, 364, 217

Dithal, S. et al. 2012, AJ, 143, 67

Due, C., Ma, J., Wu, Z. \& Zhou, X. 2006 MNRAS, 372, 1304

Fowler, A. 1904. The Observatory, 27, 197

Gizis, J. E. 1997, AJ, 113, 806 (G97)

Gizis, J. E., \& Harvin, J. 2006, AJ, 132, 2372

Goudfrooij, P., Bohlin, R. C., Walsh, J. R., Baum, S. A., 1998, STIS Instrument Science Report 98-19 
Gray, R. O., Corbally, C. J. Stellar Spectral Classification. Princeton: Princeton Univerty Press, 2009.

Halbwachs, J. L., Mayor, M., Udry, S., Arenou, F. 2003, A\&A, 397, 159

Henry, T.J., Kirkpatrick, J.D., \& Simons, D.A. 1994, AJ, 108, 1437

Iwanowska, W., \& Wayman, P.A. 1952, ApJ, 115, 129

Jao et al. 2009, ApJ, 137, 3800

Jones, E. M. 1972, ApJ, 173, 671

Joy, A. H. 1947, ApJ, 105, 96

Johnson, J. A., \& Apps, K. 2009, ApJ, 699, 933

Kirkpatrick, J.D. 1991, Ph.D. Thesis, Univ. Arizona

Kirkpatrick, J.D., Henry, T.J., \& Simons, D.A. 1995, AJ, 109, 797

Kuiper, G.P. 1938, ApJ, 87, 592

Kuiper, G.P. 1940, Apj, 91, 269

Kuiper, G.P. 1942. ApJ, 95, 201

Latham, D.W., Tonry, J., Bahcall, J. N., Soneira, R.M., \& Schechter, P. 1984, ApJ, 281, L41

Laughlin, G., Bodenheimer, P., \& Adams, F. F. 1997, ApJL, 482, 420

Lee, Y.S., et al. 2008a, AJ, 136:2022-2049

Lee, Y.S., et al. 2008b, AJ, 136:2050-2069

Lee, Y.S, et al. 2011, AJ, 141:90

Leggett, S. K., Allard, F., \& Hauschildt, P. H. 1998, ApJ, 509, 836

Lépine, S., Rich, R. M., \& Shara, M. M. 2003, AJ, 125, 1598

Lepine, S., Rich, R. M., \& Shara, M. M. 2003b, ApJ, 591, L49

Lepine, S., Shara, M. M., \& Rich, R. M. 2003c, ApJ, 585, L69

Lepine, S., Shara, M. M., \& Rich, R. M. 2004, ApJ, 602, L125

Lépine, S. 2005, Aj, 130, 1247

Lépine, S., \& Shara, M. M. 2005, AJ, 129, 1483

Lépine, S. Rich, R. M., \& Shara, M. M. 2007, ApJ, 669, 1235

Lépine, S. 2008, arXiv:0803.4203v2

Lépine, S. 2009, AIP Conf. Proc., 1094, p. 545

Lépine, S., Hilton, E.J., Mann, A.W., Wilde, M., Rojas-Ayala, B., Cruz, K.L. 2012, arxiv:1206.5991v1

Liebert, J., \& Probst, R. G. 1987, ARA\&A, 25, 473

Lundmark, K., \& Luyten, W.J. 1923, AJ, 35, 93

Luyten, W.J. 1923a, PASP, 35, 175 
Luyten, W.J. 1923b, PASP, 35, 209

Luyten, W. J. 1979, NLTT Catalogue I, II, (Minneapolis, Minnesota)

Luyten, W. J. 1980, NLTT Catalogue III, IV (Minneapolis, Minnesota)

Luyten, W. J. 1987, The LDS Catalogue: Double Stars with Common Proper Motion, Publ. Astr.

Obs. Univ. Minnesota III, part 3, 35; Proper motion survey with the 48-inch Schmidt Telescope, XXI,

XXV, XIX, XL, L, LXIV, LV, LXXI, Univ. Minnes. (1940-1987)

Luyten, W. J. 1988, Ap\&SS, 142, 17

López-Morales, M. 2007, ApJ, 660, 732

Mallada, E. H., \& Fernandez, J. A. 2001, RevMexAA, 11, 27

Monet, D. G., et al. 1992, AJ, 103, 638

Monet, D. G., et al. 2000, AJ, 120, 1541

Morgan, W.W. 1938. ApJ, 87, 589

Morgan, W.W., Keenan, P.C., \& Kellman, E. 1943, An Atlas of Stellar Spectra, Chicago: University of Chicago Press

Mould, J. R., \& Hyland, A. R. 1976, ApJ, 208, 399

Neves, V., Bonfils, X., Santos, N.C., et al. 2012, A\&A, 538, A25

Öhman, Y. 1934, Apj, 80, 171

Öhman, Y. 1936a, Stockholms Observatoriums Annaler, 12, No.3

Öhman, Y. 1936b, Stockholms Observatoriums Annaler, 12, No.8

Phan-Bao, N., \& Bessel, M. S. 2006, A\&A, 446, 515

Pickering, E.C, 1890, Annals of Harvard College Observatory, 27, 1

Prieto, C.A., et al. 2008, AJ, 136:2070-2082

Reid, I. N., Hawley, S., \& Gizis, J. E. 1995, AJ, 110, 1838

Reid, I. N., \& Gizis, J. E. 2005, PASP, 117, 676

Retterer, J. M., \& King, I. R. 1982, ApJ, 254, 214

Rojas-Ayala, B., et al. 2010, ApJ, 720, L113

Rossi, S., Beers, T.C., Sneden, C., et al. 2005, AJ, 130, 2804

Salim, S., \& Gould, A. 2002, ApJ, 575, L83

Schalauffman, K.C., \& Laughlin, G. 2010, A\&A, 519, A105

Schweitzer, A., et al. 1999, A\&A, 350, L62

Scholz, R.-D., Lodieu, N., \& McCaughrean, M. J. 2004, A\&A, 428, L25

Scholz, R.-D., et al. 2004a, A\&A, 425, 519 
Secchi, A. 1866, CR Acad. Sci. Paris, 63, 621

Secchi, A. 1868, MNRAS, 28, 196

Skrutskie, M. F., et al. 2006, AJ, 131, 1163

Smolinski, J.P., et al. 2011, AJ, 141:89

Valenti, J.A., \& Fisher, D.A. 2005, VizieR Online Data Catalog, 215, 90141

Weinberg, M. D., Shapiro, S. L., \& Wasserman, I. 1987, ApJ, 312, 367

Wittkowski, M., Aufdenberg, J.P., \& Kervella, P. 2004, AA, 413, 711

Wittkowski, M., Aufdenberg, J.P., Driebe, T., Roccataglita, V., Szeifert, T., \& Wolff, B. 2006b, AA, 460,855

Wittkowski, M., Hummel, C.A., Aufdenberg, J.P., \& Roccatagliata, V. 2006a, AA, 460, 843

Woolf, V. M., \& Wallerstein, G. 2006, PASP, 118, 218

Woolf, V. M., Lépine, S., \& Wallerstein, G. 2009, PASP, 121, 117 
Apêndice 

Apêndice $\mathrm{A}$

Resultados para toda amostra observada 
Tabela A.1 - Resultados para $[\mathrm{Fe} / \mathrm{H}]$ e $[\mathrm{C} / \mathrm{Fe}]$ obtido pelo n-sspp para todos os pares observados. $\mathrm{O}$ incerteza nas abundâncias é de $0.2 \mathrm{dex}$

\begin{tabular}{|c|c|c|c|c|c|c|}
\hline Par & Primária & Abundâ & mpartilhadas & Secundári & & Sistema \\
\hline Identificação & Nome & {$[\mathrm{Fe} / \mathrm{H}]$} & {$[\mathrm{C} / \mathrm{Fe}]$} & Nome & $\mathrm{S} / \mathrm{N}>20$ & $\mathrm{~K}_{\text {index }}$ \\
\hline 1 & PM_I00471-2512W & 0.04 & -0.14 & PM_I00471-2514N & não & 1.8 \\
\hline 2 & PM_I01008-2125 & -0.88 & 0.75 & PM_I01008-2126 & $\operatorname{sim}$ & 1.1 \\
\hline 3 & PM_I00426-2315 & -0.92 & 0.81 & PM_I00426-2314 & $\operatorname{sim}$ & 1.9 \\
\hline 4 & PM_I01266-4842W & -1.37 & 1.21 & $\mathrm{PM}_{-} \mathrm{I} 01266-4842 \mathrm{E}$ & $\operatorname{sim}$ & 1.4 \\
\hline 5 & PM_I01543-2249S & -0.61 & 0.66 & PM_I01543-2249N & $\operatorname{sim}$ & 1.6 \\
\hline 6 & PM_I02300-2402W & 0.32 & -0.48 & PM_I02299-2401Ss & não & 1.8 \\
\hline 6 & PM_I02300-2402W & 0.32 & -0.48 & PM_I02300-2402E & $\operatorname{sim}$ & 2.5 \\
\hline 7 & PM_I02463-2312 & -0.45 & 0.35 & PM_I02463-2312 & $\operatorname{sim}$ & 2.2 \\
\hline 8 & PM_I02542-6620 & -0.57 & 1.09 & PM_I02542-6619 & $\operatorname{sim}$ & 1.1 \\
\hline 9 & PM_I04325-5657N & -0.92 & 0.79 & PM_I04325-5657S & $\operatorname{sim}$ & 1.3 \\
\hline 10 & PM_I04477-3044W & -0.84 & 0.80 & $\mathrm{PM}_{-} \mathrm{I} 04477-3044 \mathrm{E}$ & $\operatorname{sim}$ & 2.2 \\
\hline 11 & PM_I05418-5000 & -0.27 & 0.16 & PM_I05418-5002 & não & 1.5 \\
\hline 12 & PM_I05425-4305E & -0.58 & 0.45 & PM_I05425-4305W & $\operatorname{sim}$ & 1.3 \\
\hline 13 & PM_I05442-2416 & -0.56 & 0.43 & PM_I05442-2417N & $\operatorname{sim}$ & 1 \\
\hline 14 & PM_I0559-4511 & -0.16 & 0.11 & PM_I05560-4511 & $\operatorname{sim}$ & 1.2 \\
\hline 15 & PM_I06276-2355W & -0.22 & 0.16 & PM_I06276-2356 & $\operatorname{sim}$ & 1.2 \\
\hline 16 & PM_I08239-7549W & -0.29 & 0.20 & PM_I08239-7549E & $\operatorname{sim}$ & 1.6 \\
\hline 17 & PM_I08386-3856 & -0.75 & 0.63 & PM_I08386-3857 & $\operatorname{sim}$ & 1.5 \\
\hline 18 & PM_I13133-4153N & -1.4 & 1.24 & PM_I13133-4153S & $\operatorname{sim}$ & 1.8 \\
\hline 19 & PM_I18240-5107N & -0.54 & 0.83 & PM_I18241-5107 & $\operatorname{sim}$ & 1 \\
\hline 19 & PM_I18240-5107S & -0.33 & 0.22 & PM_I18241-5107 & $\operatorname{sim}$ & 1 \\
\hline 20 & PM_I20345-2219 & -0.86 & 1.23 & PM_I20345-2217 & não & 1.5 \\
\hline 21 & PM_I20371-5815 & -1.62 & 1.90 & PM_I20371-5816 & $\operatorname{sim}$ & 1 \\
\hline 22 & PM_I21175-4142E & -0.93 & 0.86 & $\mathrm{PM}_{-} \mathrm{I} 21175-4142 \mathrm{~W}$ & $\operatorname{sim}$ & 2.3 \\
\hline 23 & PM_I21229-7141 & -0.55 & 0.94 & PM_I21229-7142 & não & 2.4 \\
\hline 24 & PM_I21545-7102 & -0.38 & 0.24 & PM_I21547-7103 & não & 1.3 \\
\hline 25 & PM_I22487-5613W & -0.38 & 0.20 & $\mathrm{PM}_{-} \mathrm{I} 22487-5613 \mathrm{E}$ & $\operatorname{sim}$ & 1.3 \\
\hline 26 & PM_I23033-5311 & -1.19 & 1.27 & PM_I23034-5311 & $\operatorname{sim}$ & 2 \\
\hline 27 & PM_I230992057 & -1.43 & 1.51 & PM_I23099-2056 & $\operatorname{sim}$ & 2.6 \\
\hline 28 & PM_I23324-2154W & -0.97 & 1.12 & PM_I23324-2154E & não & 1.6 \\
\hline 36 & PM_I01147-4536 & 0.27 & -0.40 & não observada & - & - \\
\hline 37 & PM_I03317-2119 & -0.47 & 0.61 & não observada & - & - \\
\hline 38 & PM_I03533-2313 & -0.58 & 0.57 & não observada & - & - \\
\hline 39 & PM_I06291-2305Wn & -0.09 & -0.01 & não observada & - & - \\
\hline 40 & PM_I07194-7422 & -0.58 & 0.50 & não observada & - & - \\
\hline
\end{tabular}


Apêndice B

Espectros estelares 


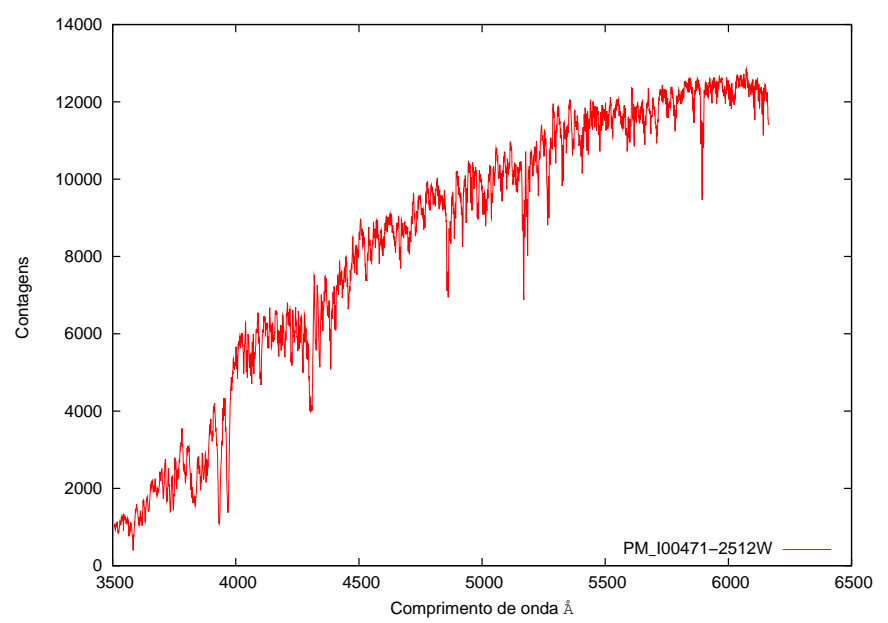

\section{$\mathrm{S} / \mathrm{N}<20$}

Figura B.1: Par 1
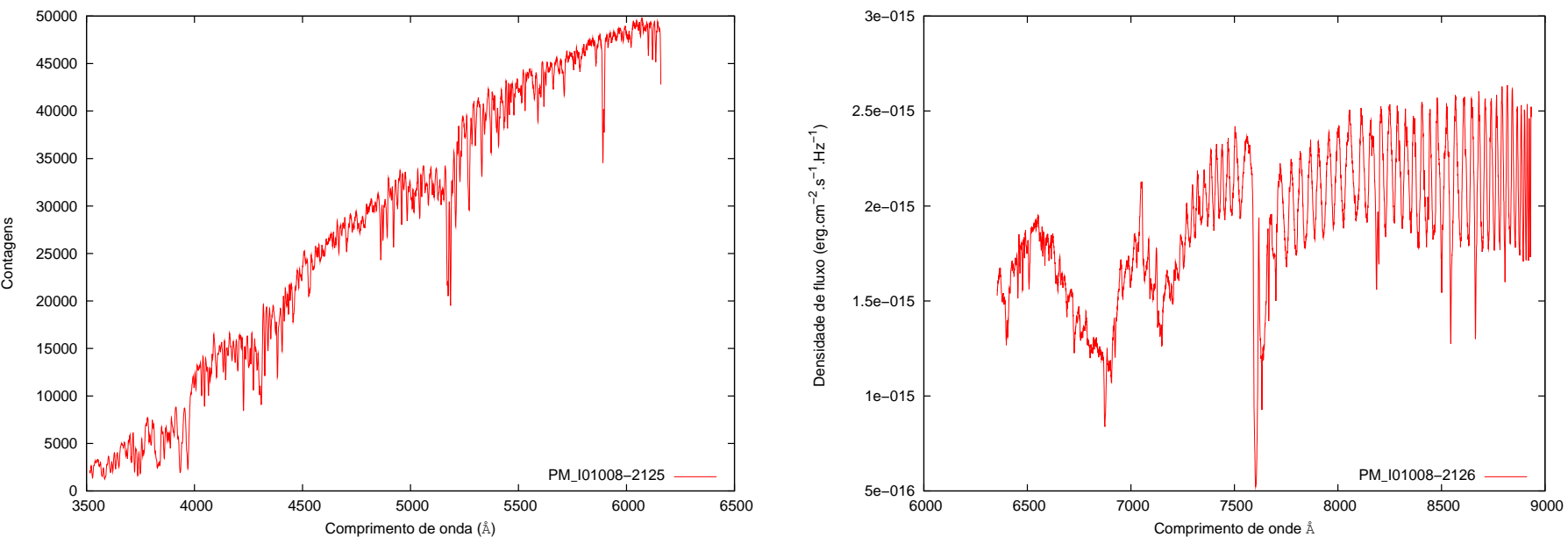

Figura B.2: Par 2
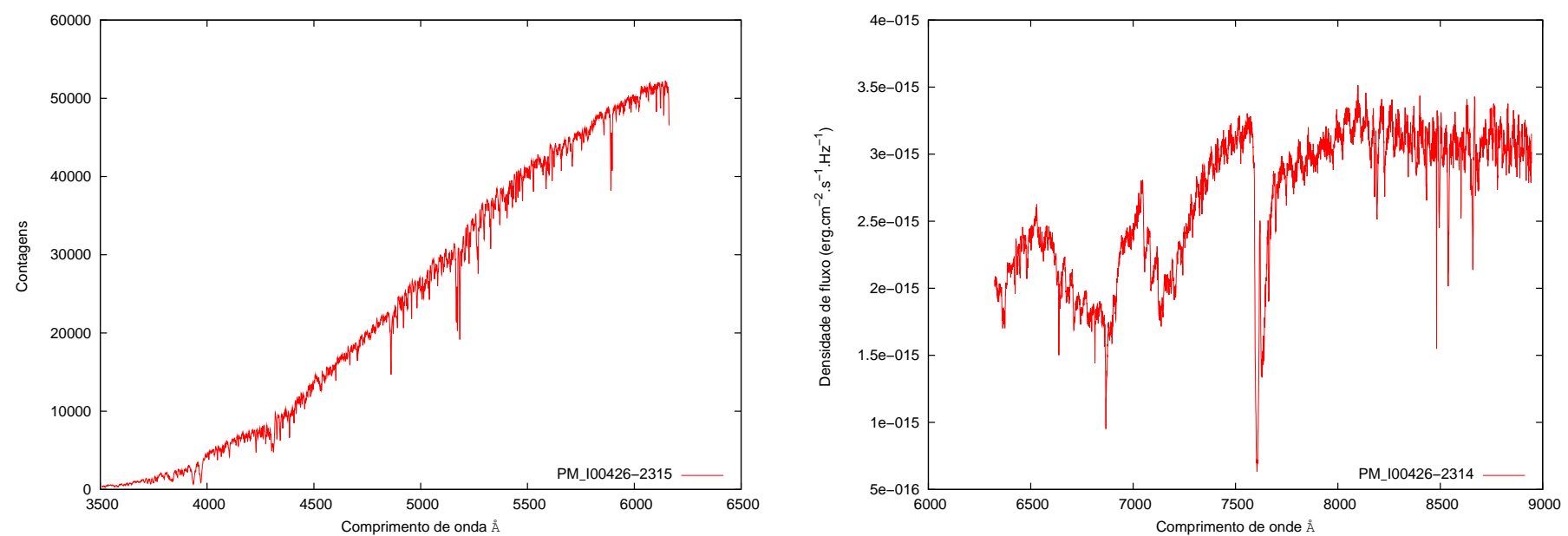

Figura B.3: Par 3 

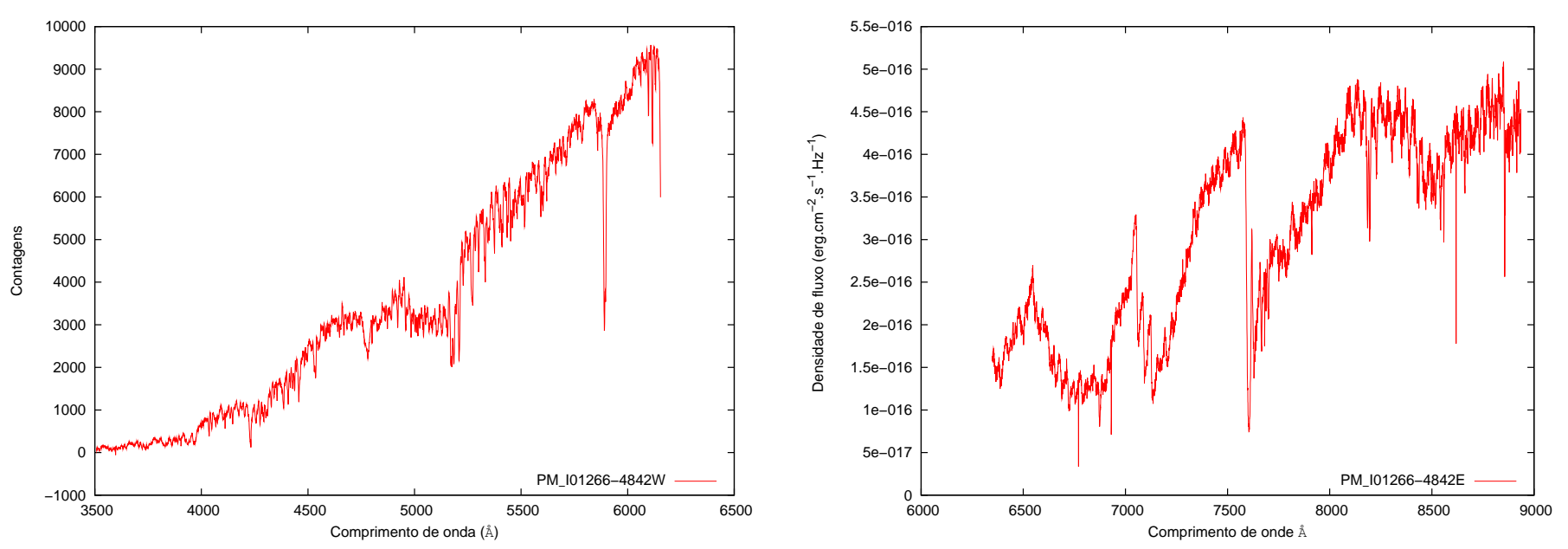

Figura B.4: Par 4
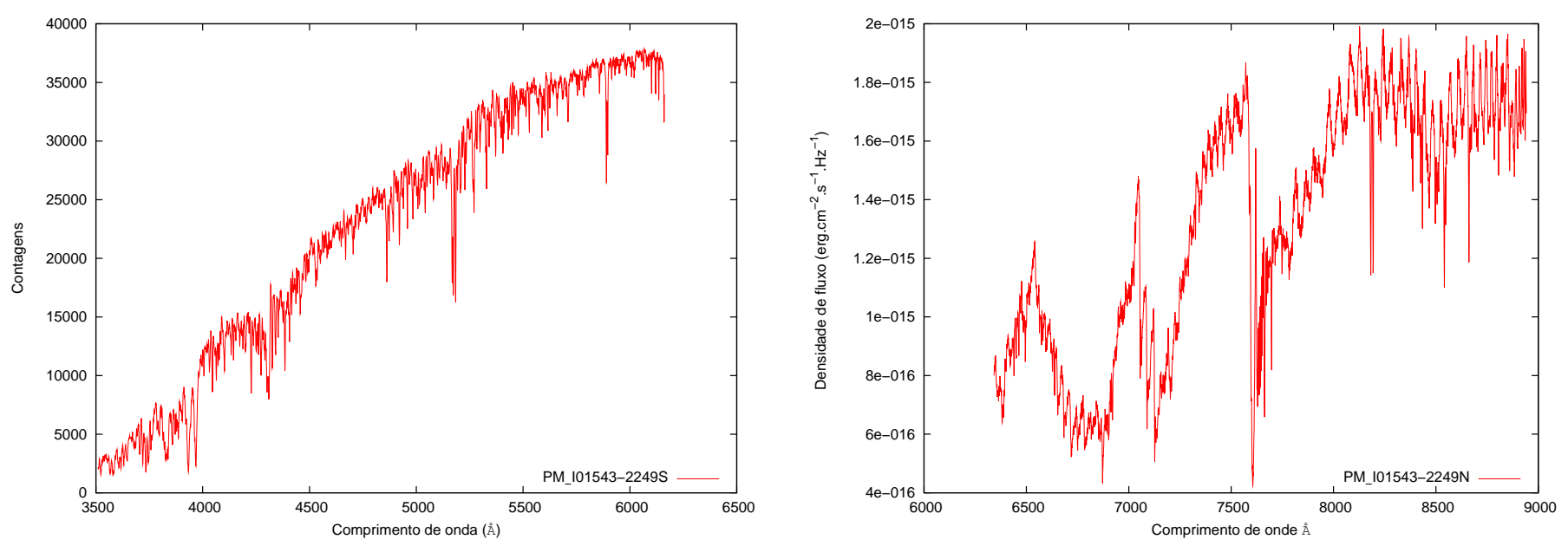

Figura B.5: Par 5

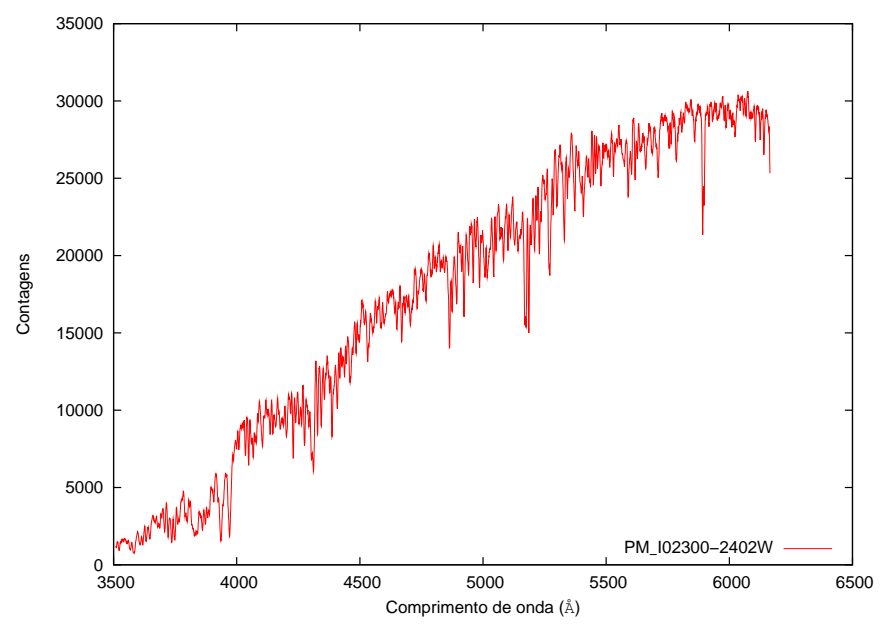

$\mathrm{S} / \mathrm{N}<20$

Figura B.6: Par 6 

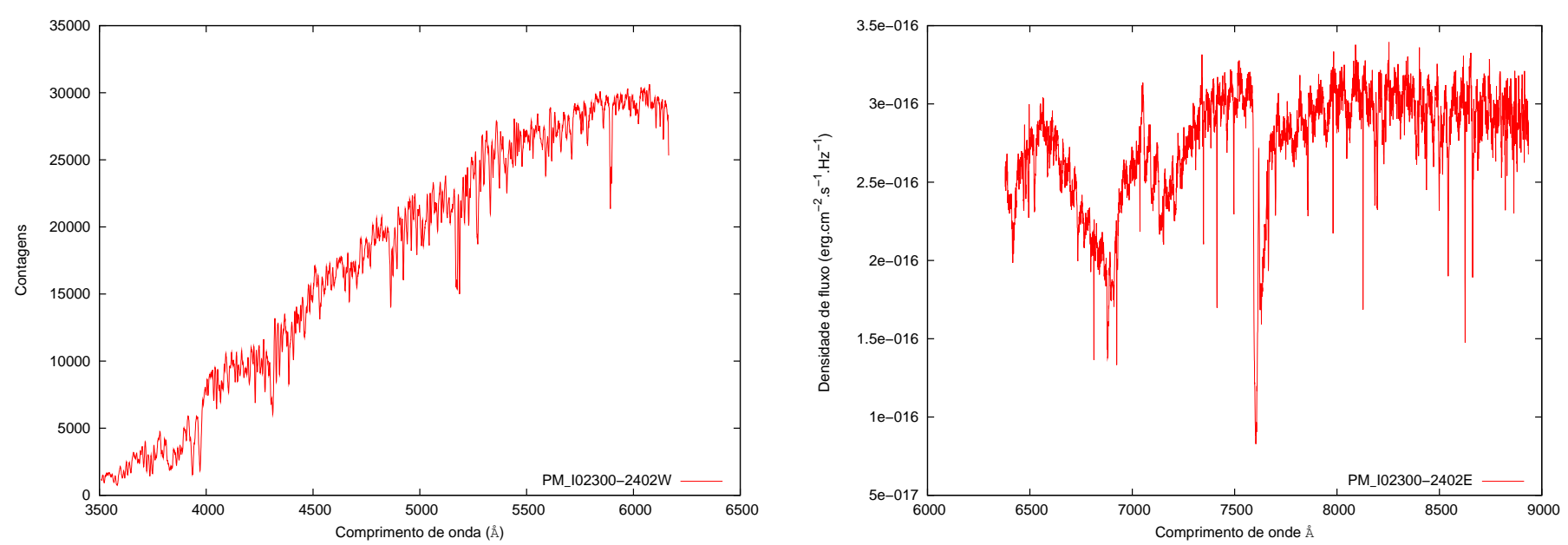

Figura B.7: Par6
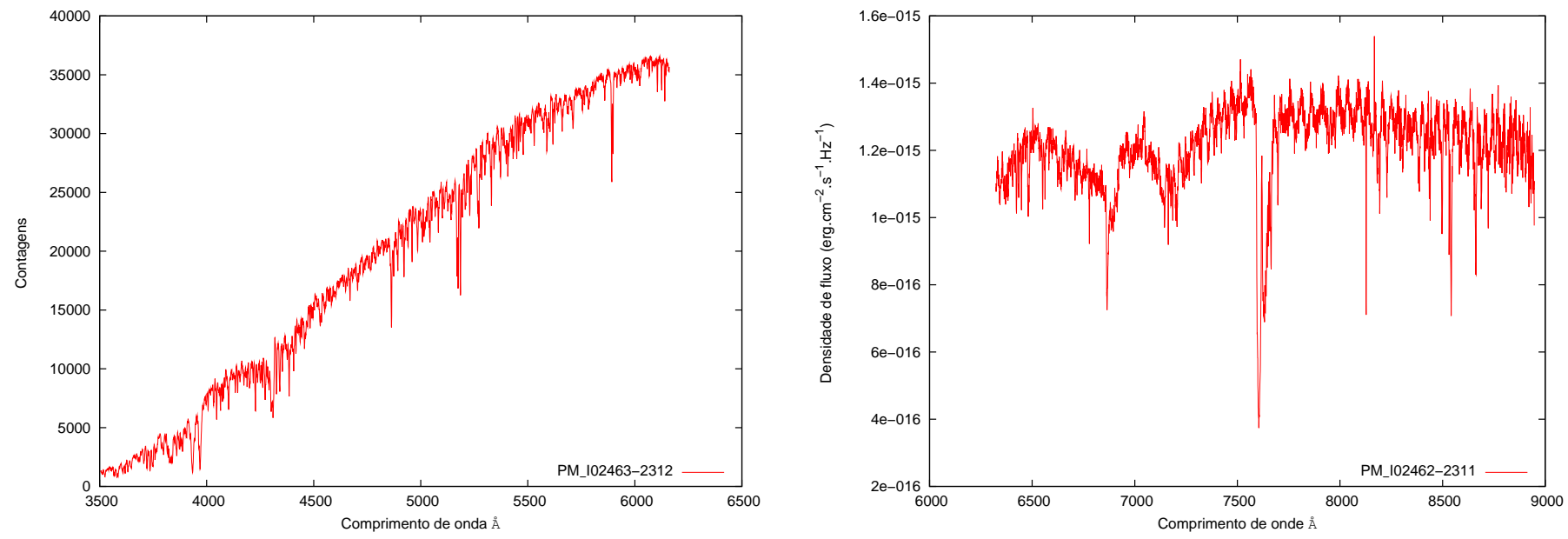

Figura B.8: Par 7
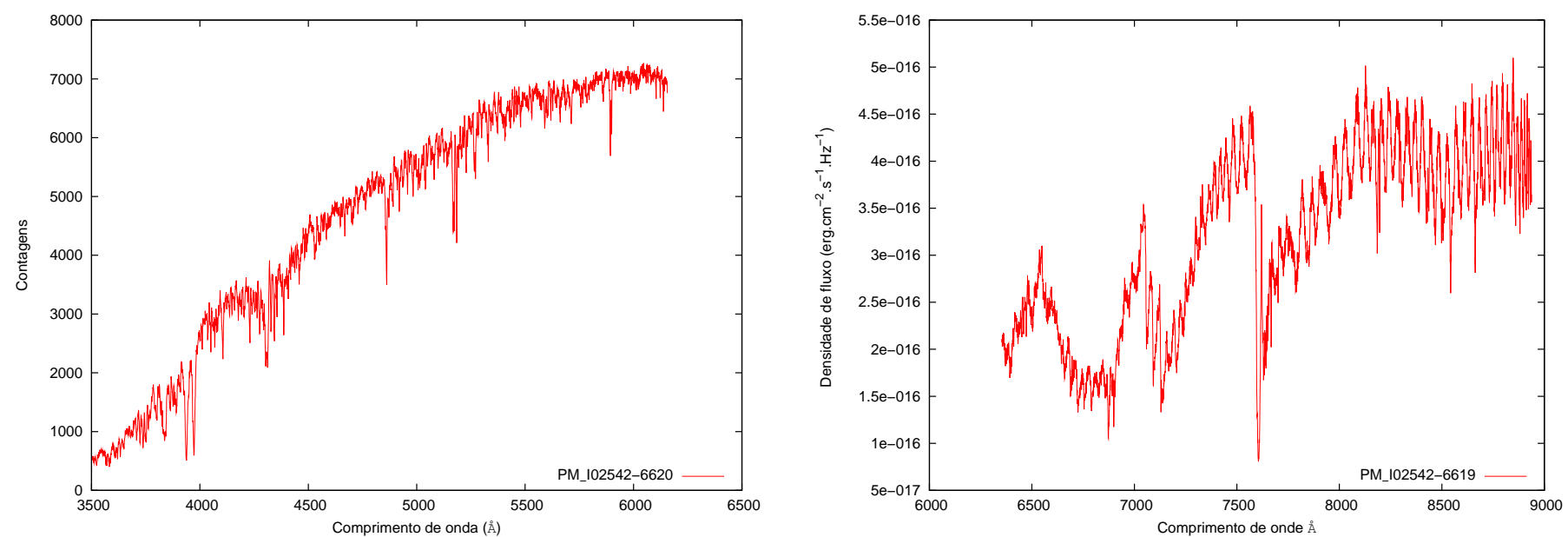

Figura B.9: Par 8 

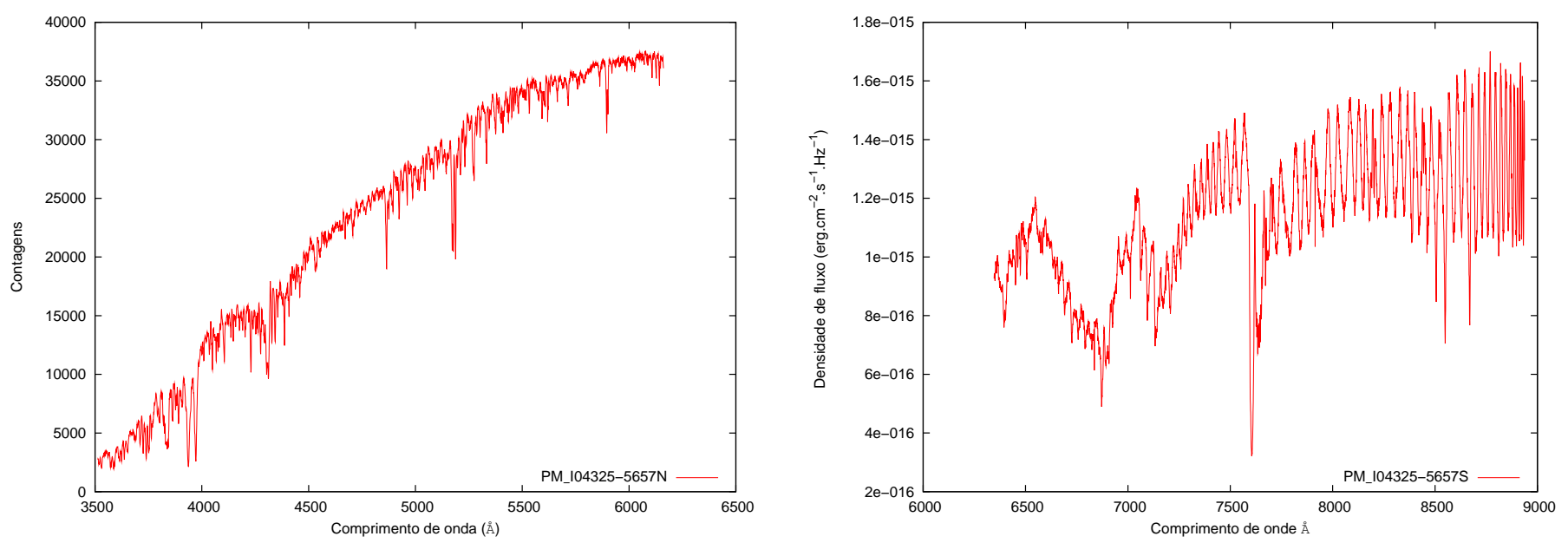

Figura B.10: Par 9
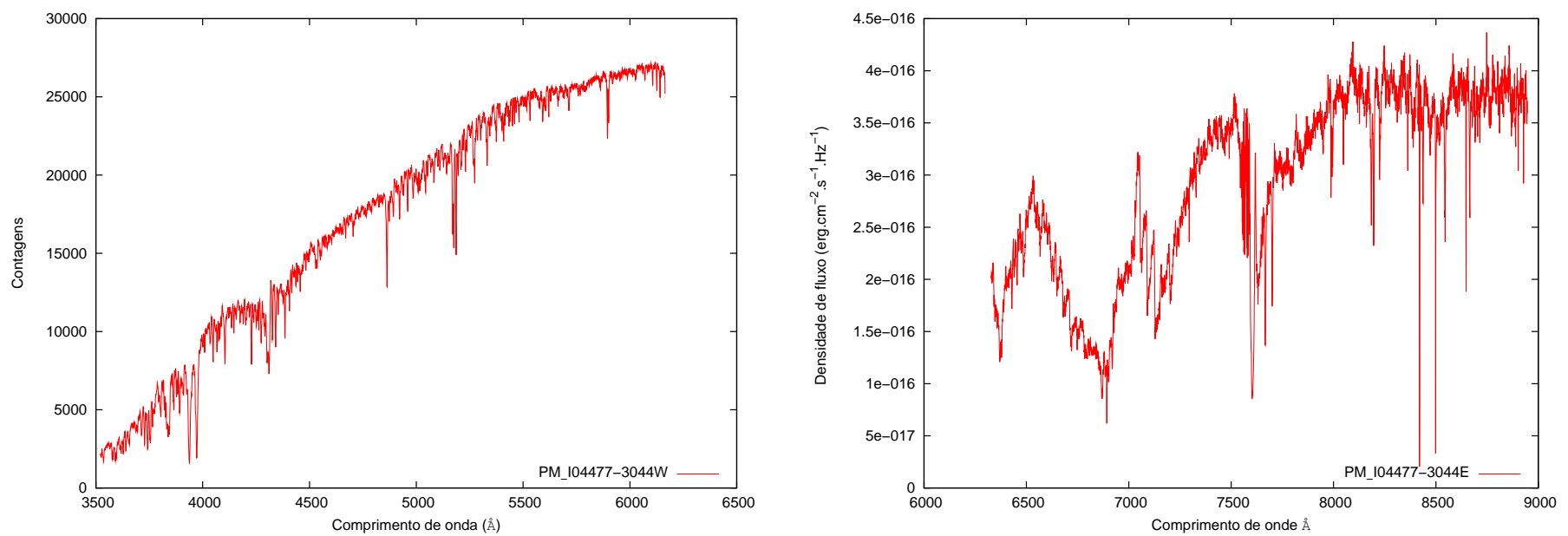

Figura B.11: Par 10

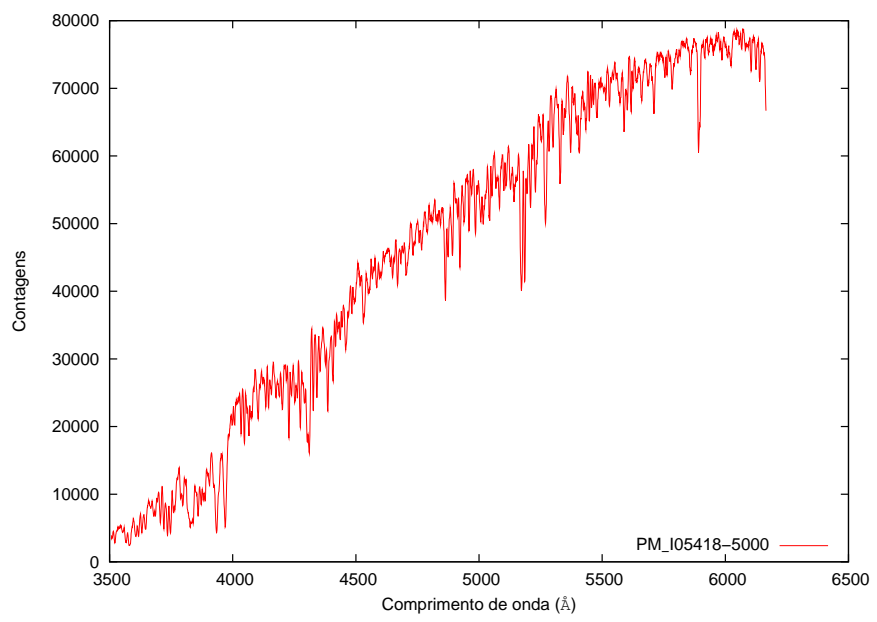

$\mathrm{S} / \mathrm{N}<20$

Figura B.12: Par 11 

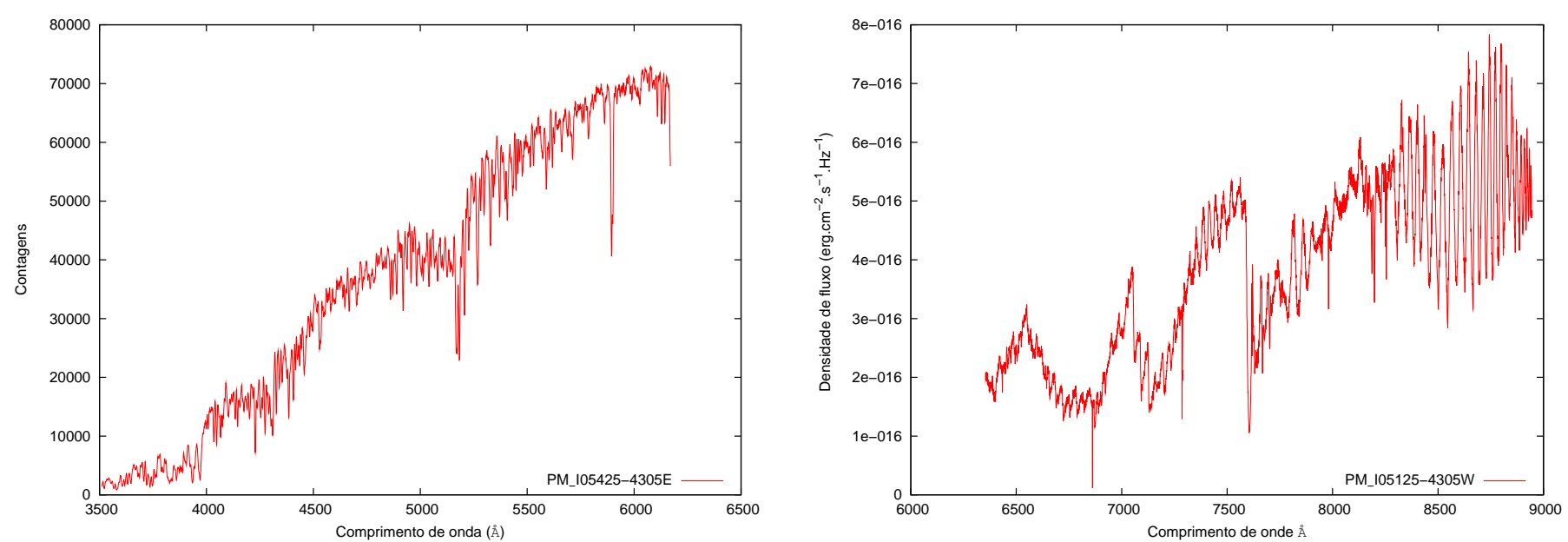

Figura B.13: Par 12
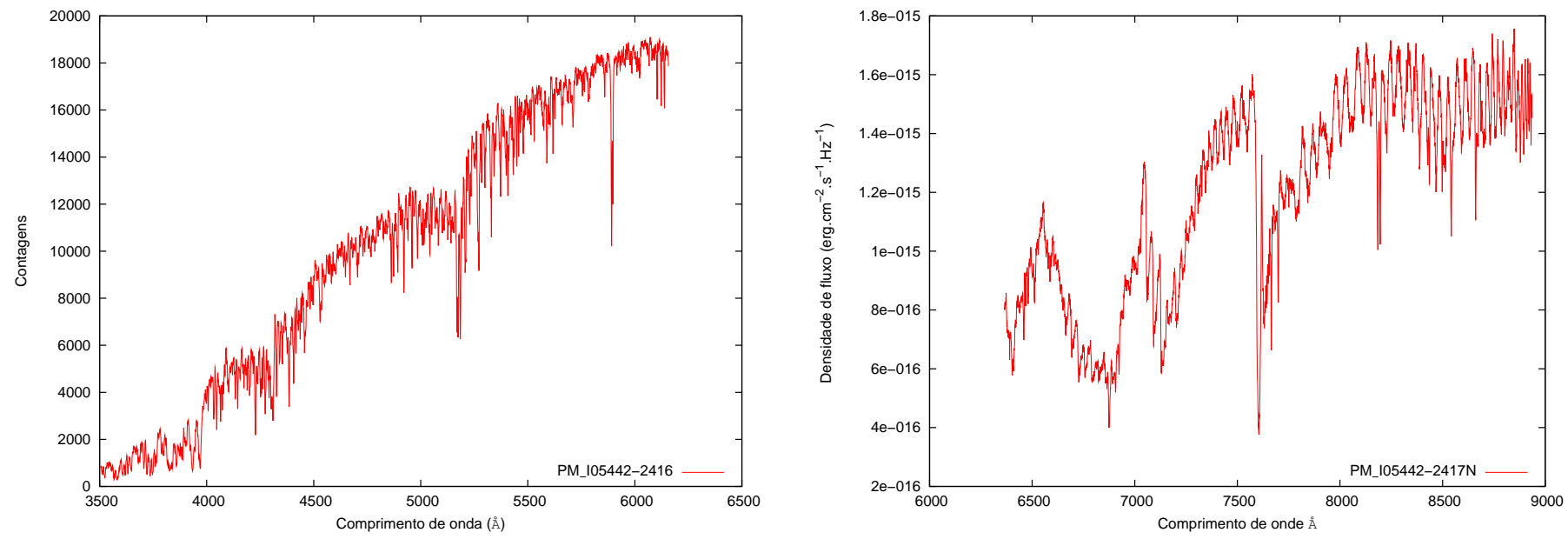

Figura B.14: Par 13
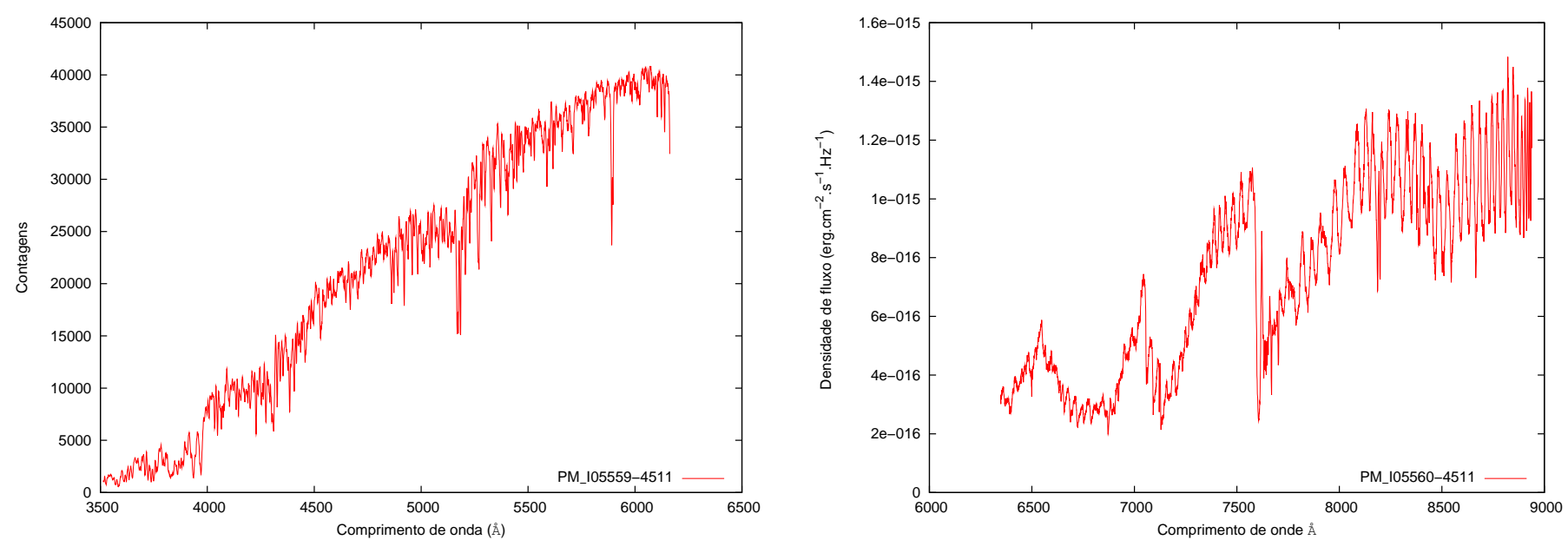

Figura B.15: Par 14 

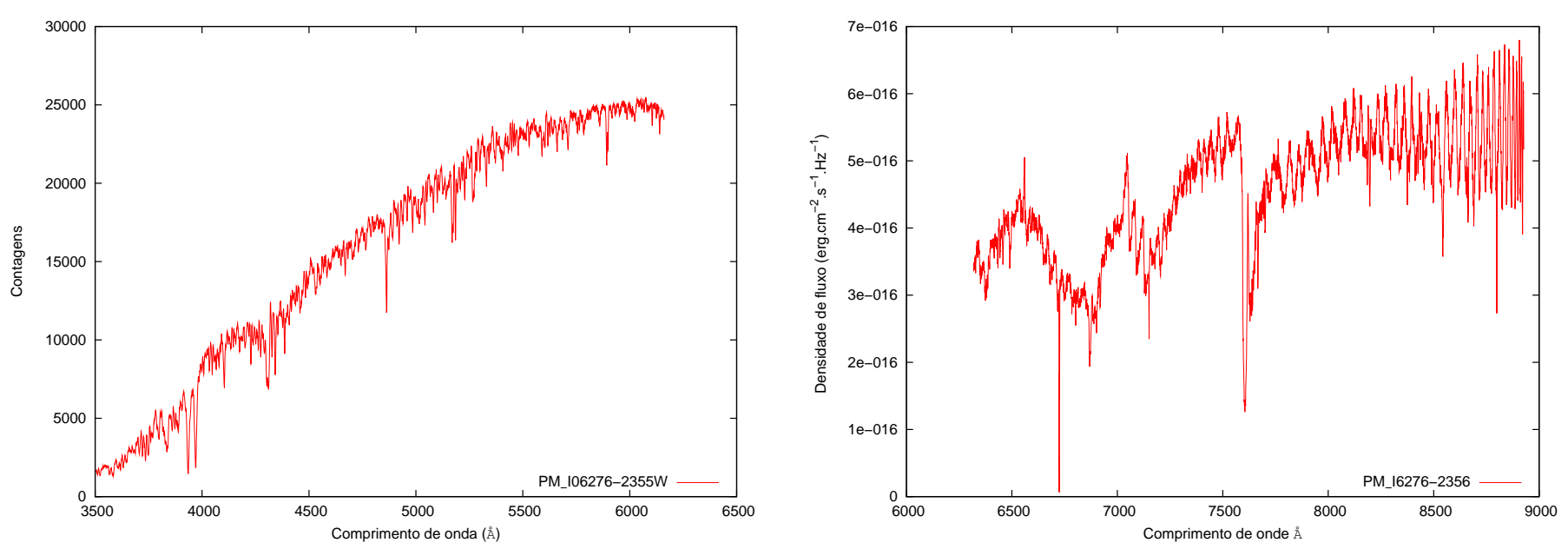

Figura B.16: Par 15
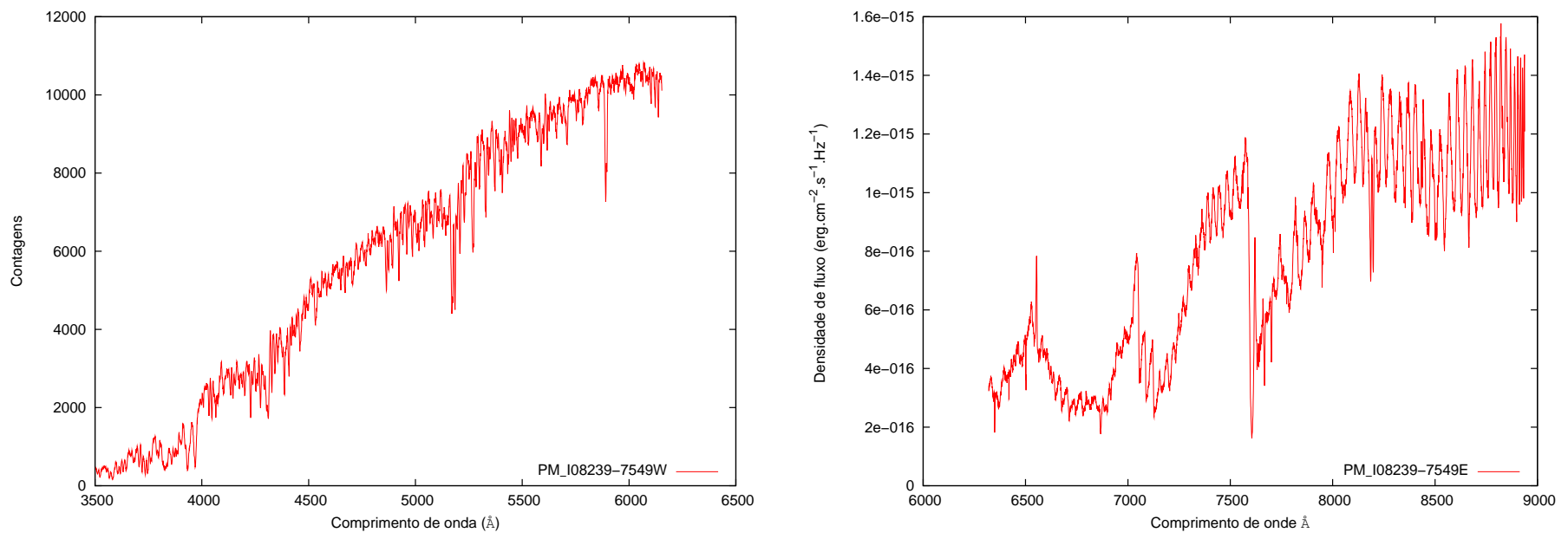

Figura B.17: Par 16
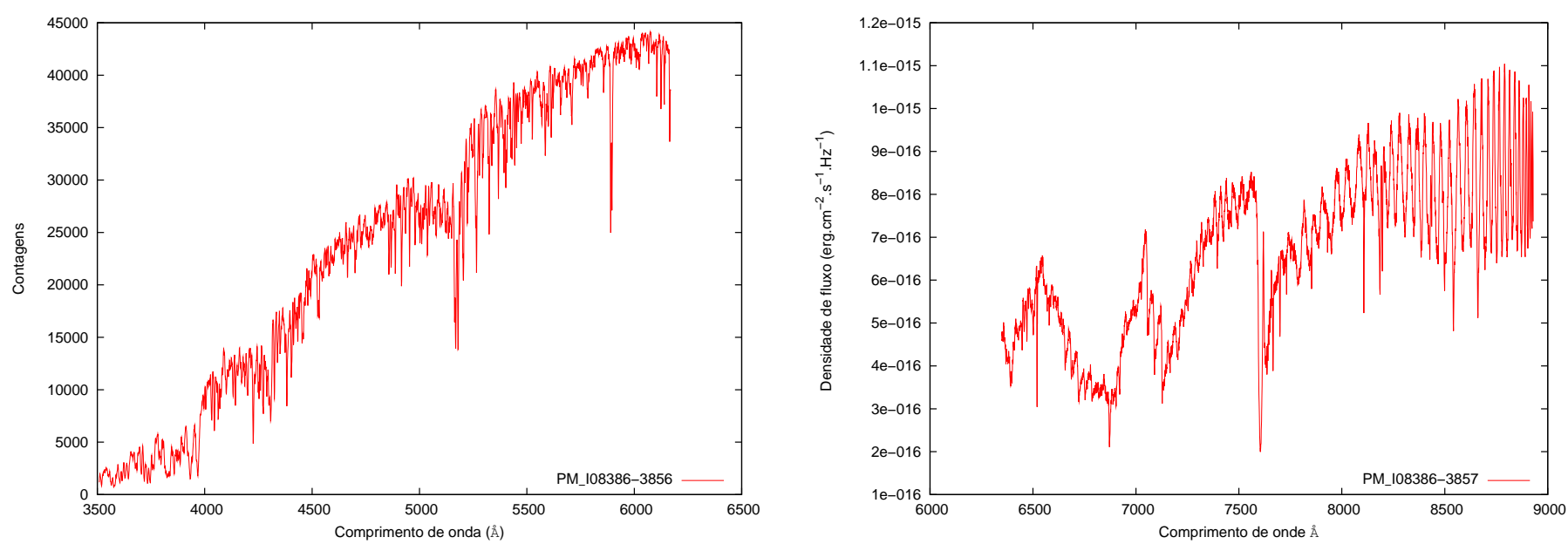

Figura B.18: Par 17 

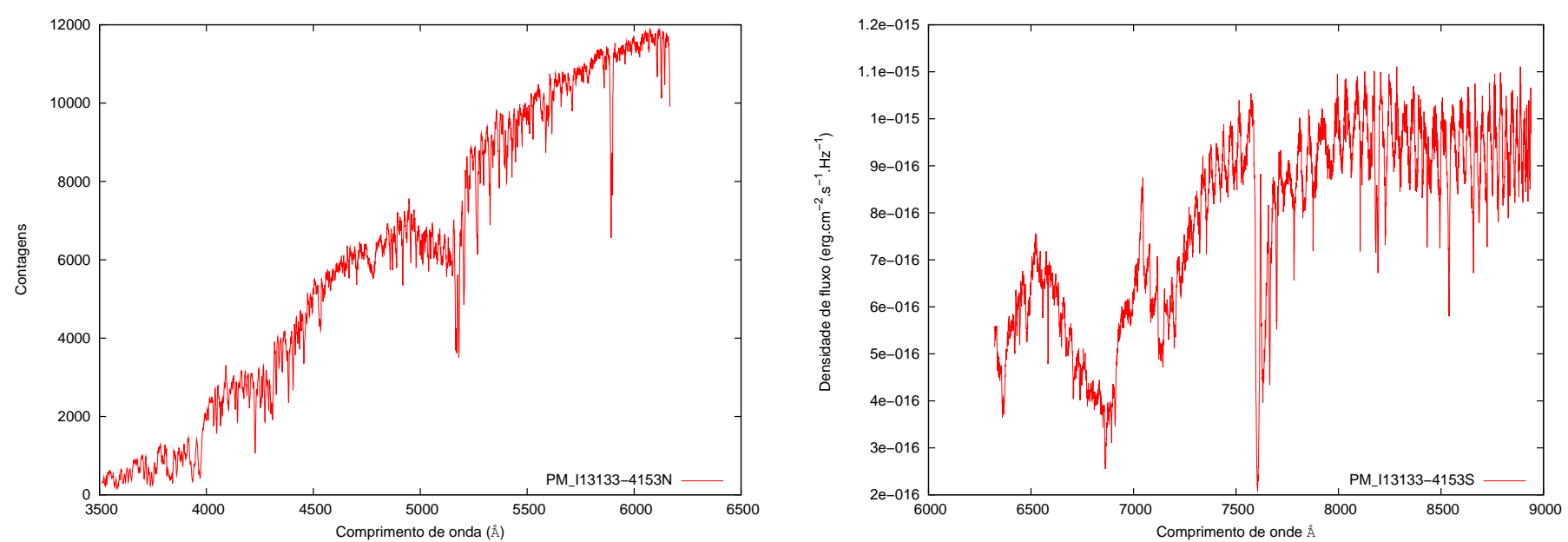

Figura B.19: Par 18
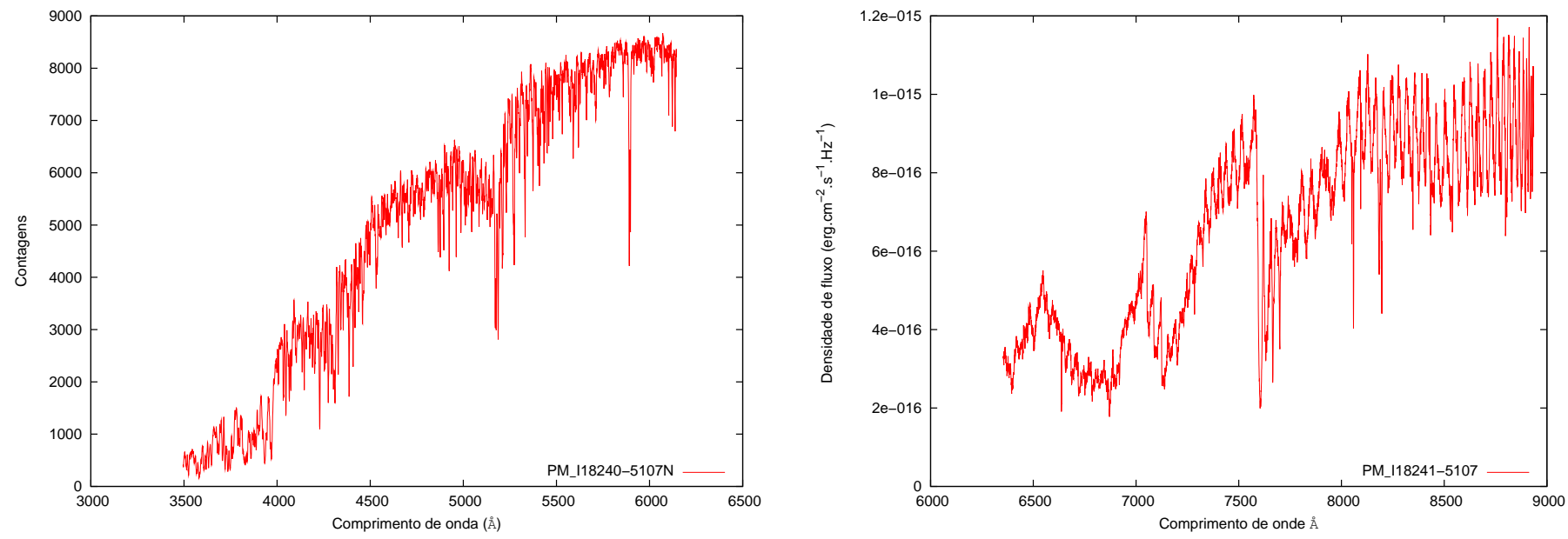

Figura B.20: Par 19
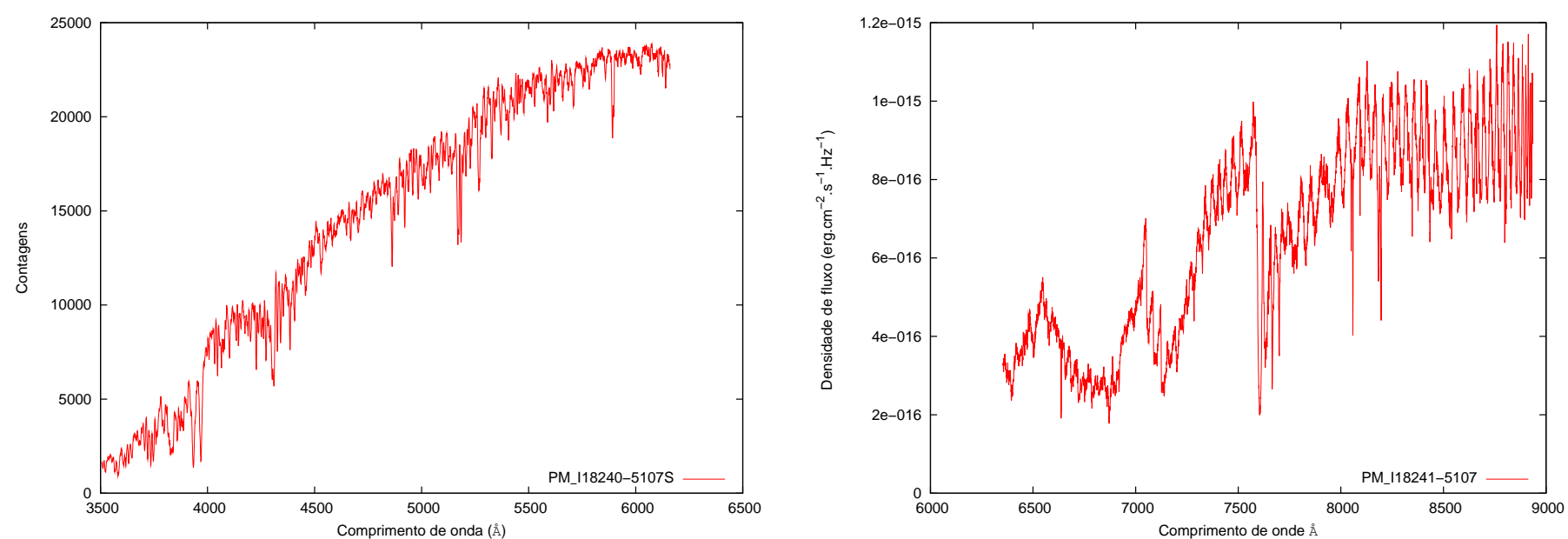

Figura B.21: Par 19 


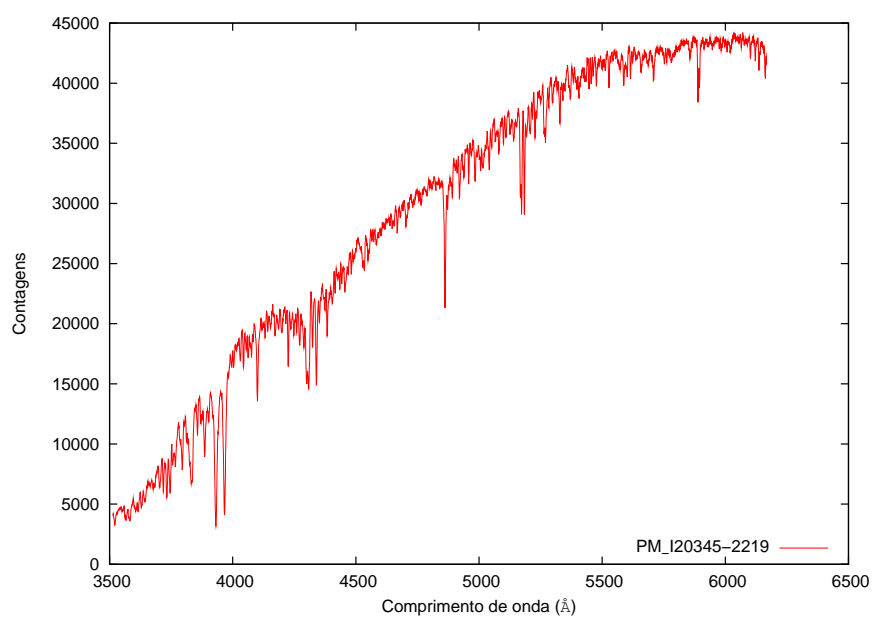

$\mathrm{S} / \mathrm{N}<20$

Figura B.22: Par 20
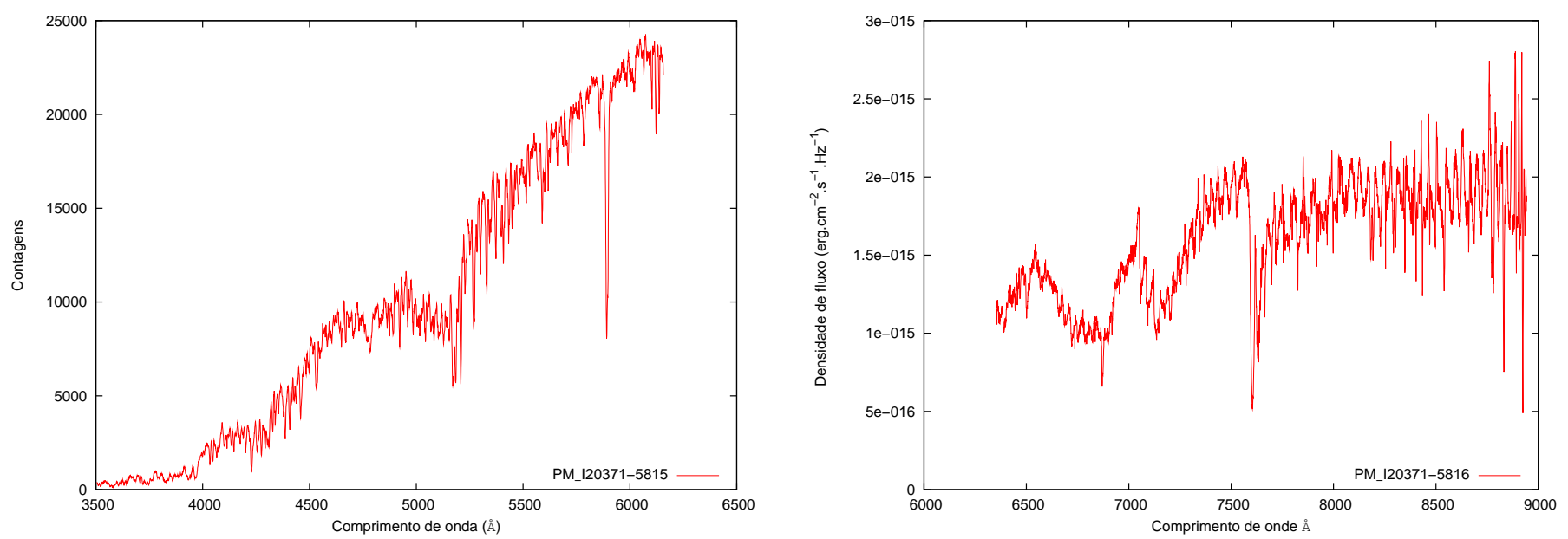

Figura B.23: Par 21
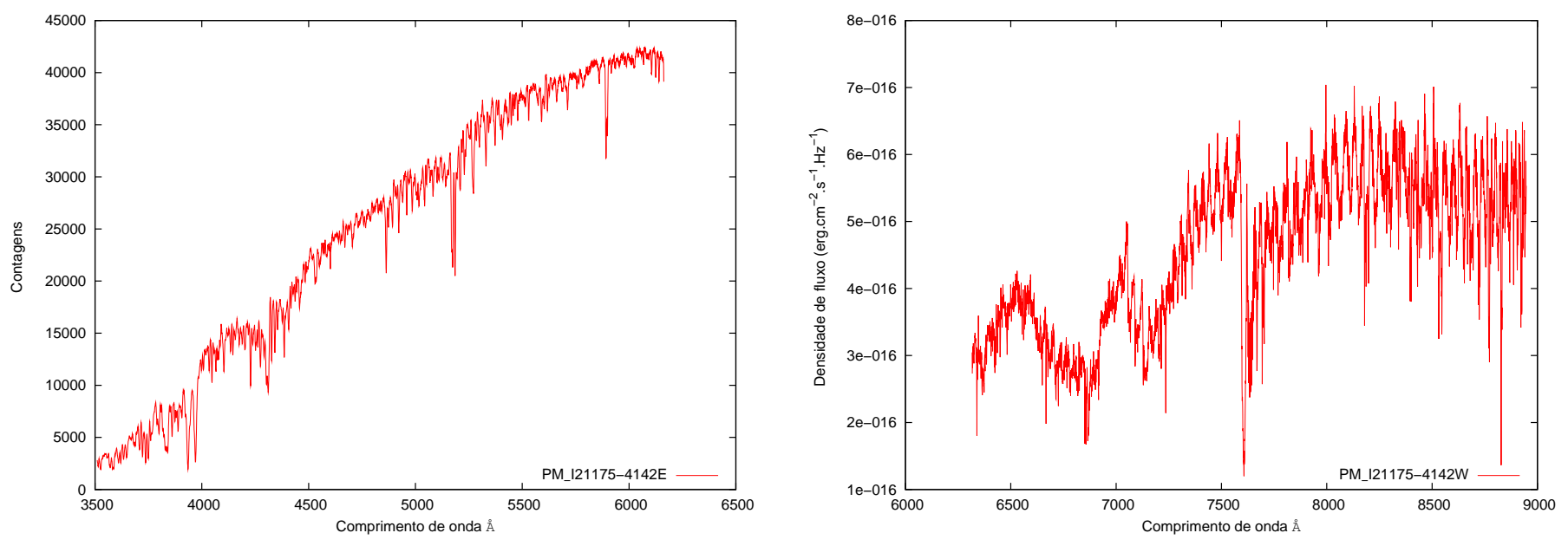

Figura B.24: Par 22 


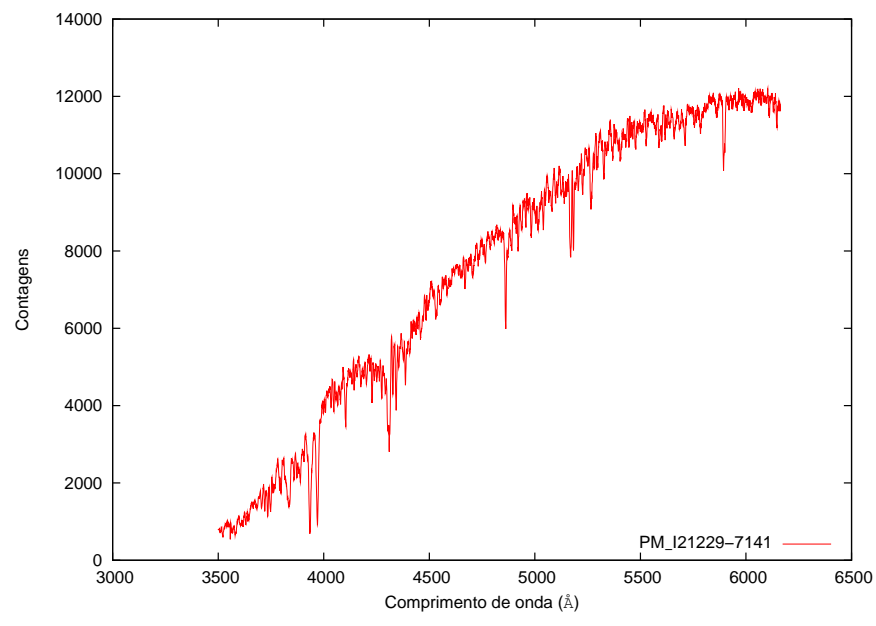

\section{$S / N<20$}

Figura B.25: Par 23

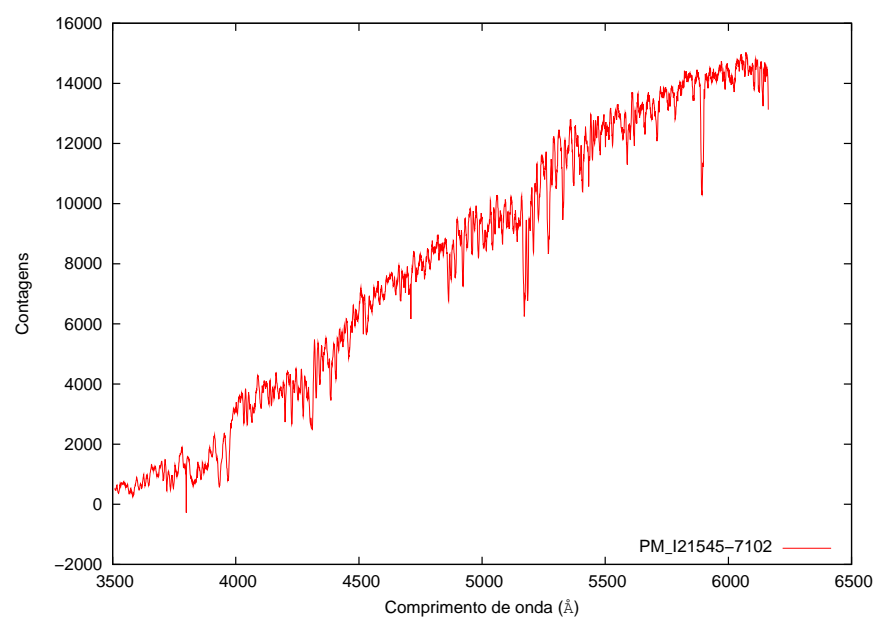

\section{$\mathrm{S} / \mathrm{N}<20$}

Figura B.26: Par 24
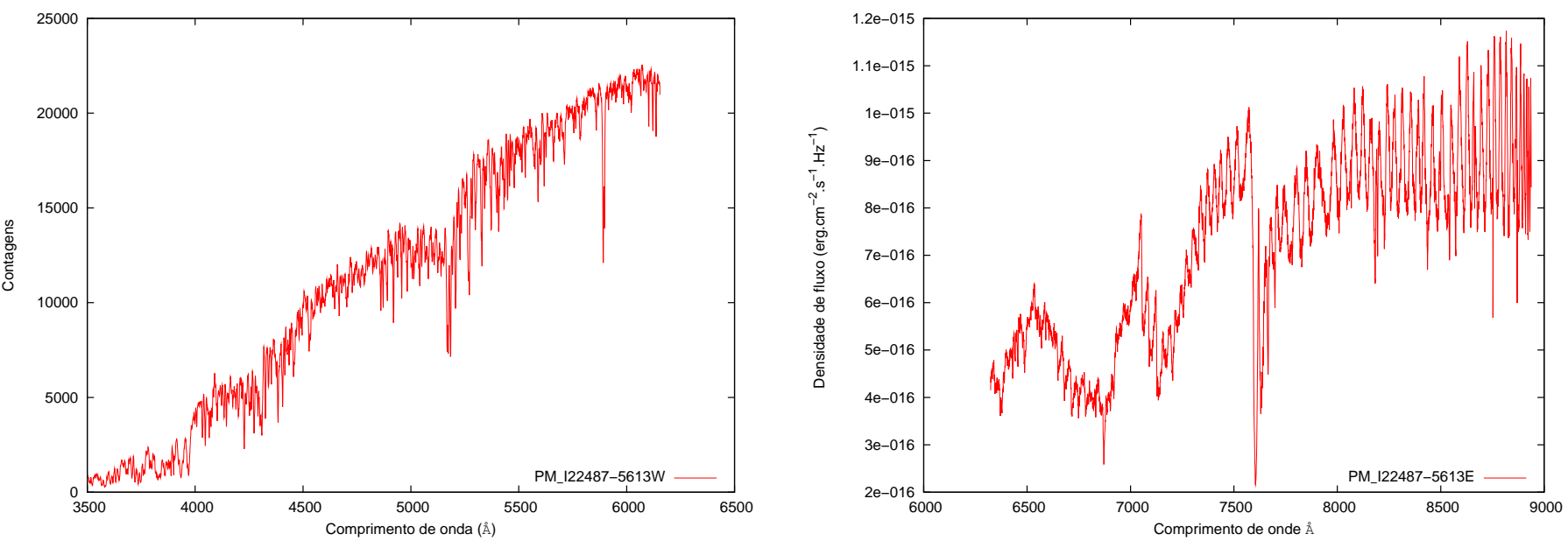

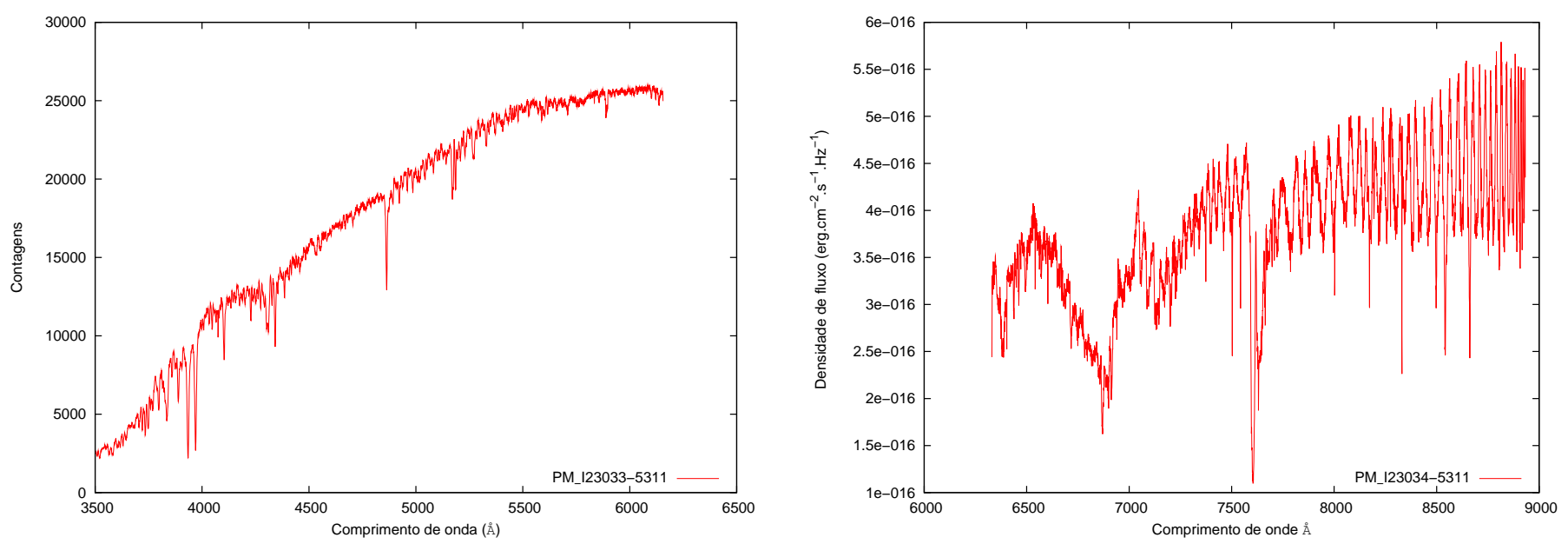

Figura B.28: Par 26
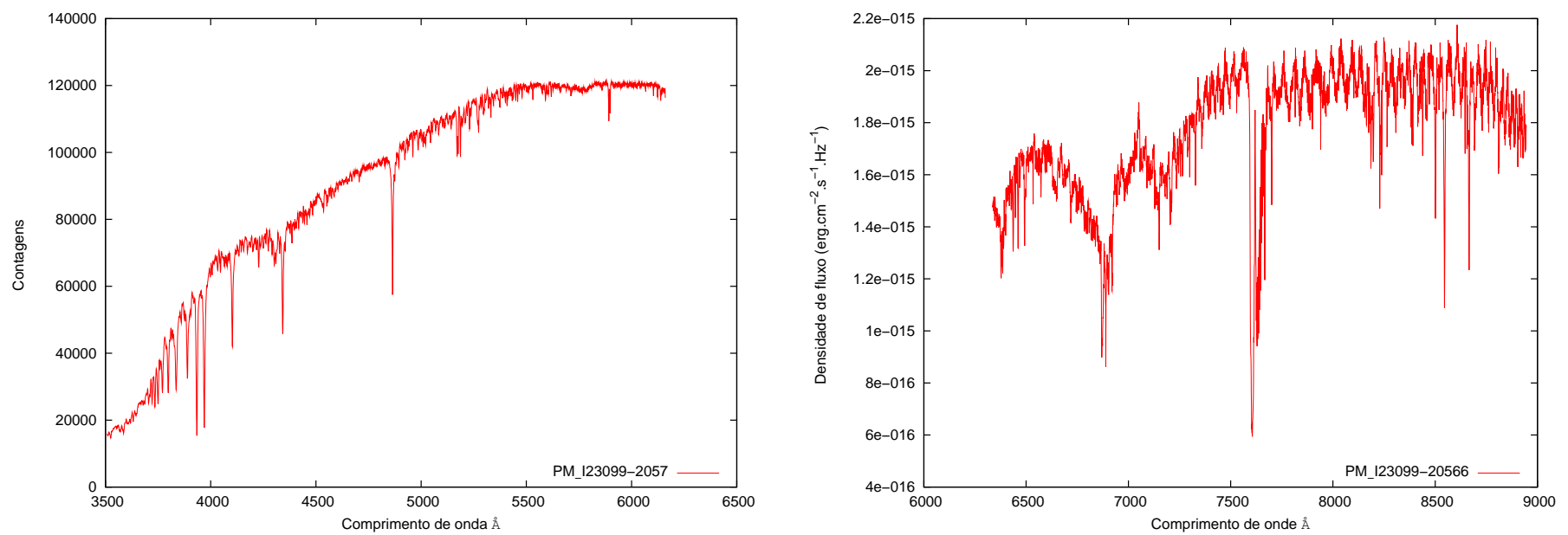

Figura B.29: Par 27

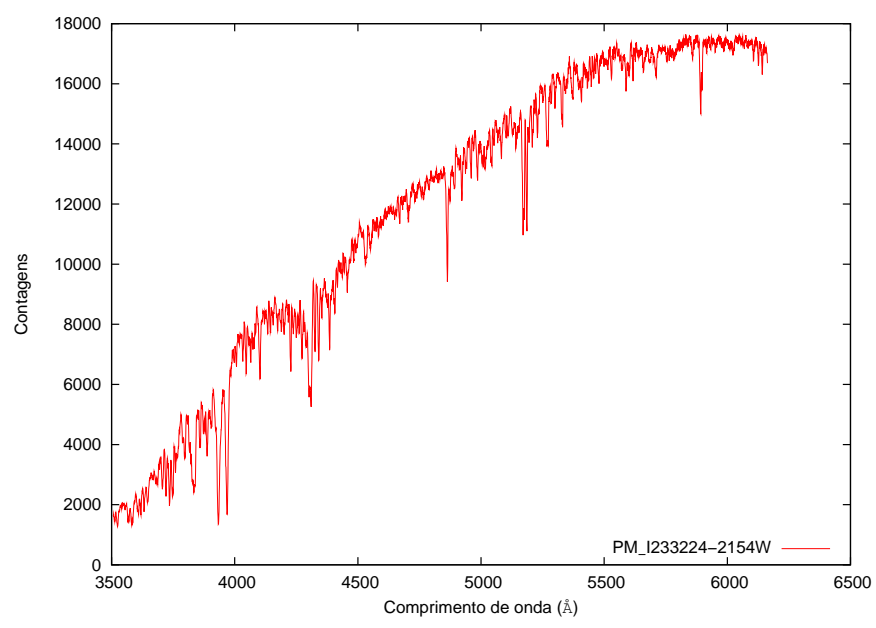

$\mathrm{S} / \mathrm{N}<20$

Figura B.30: Par 28 


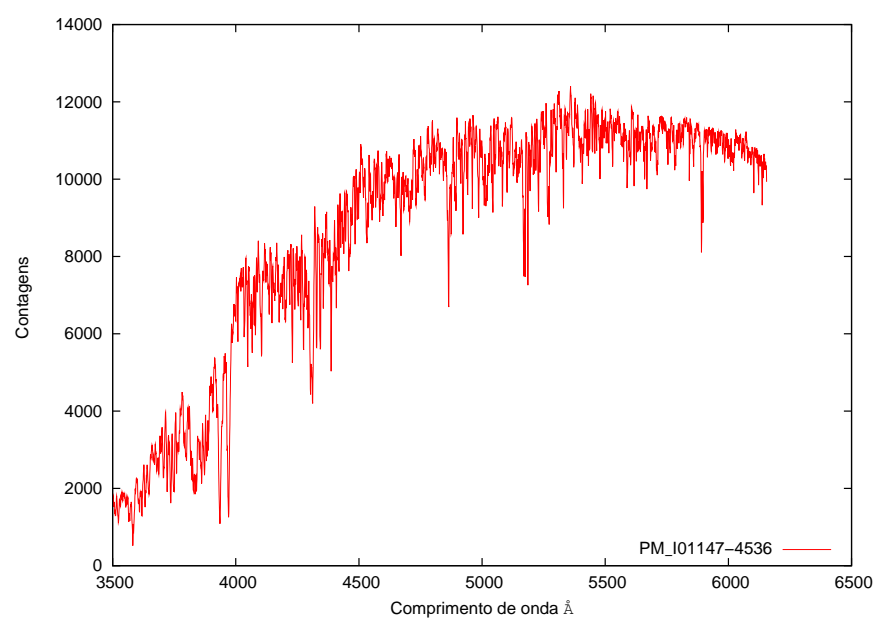

\section{Não observada}

Figura B.31: Par 36

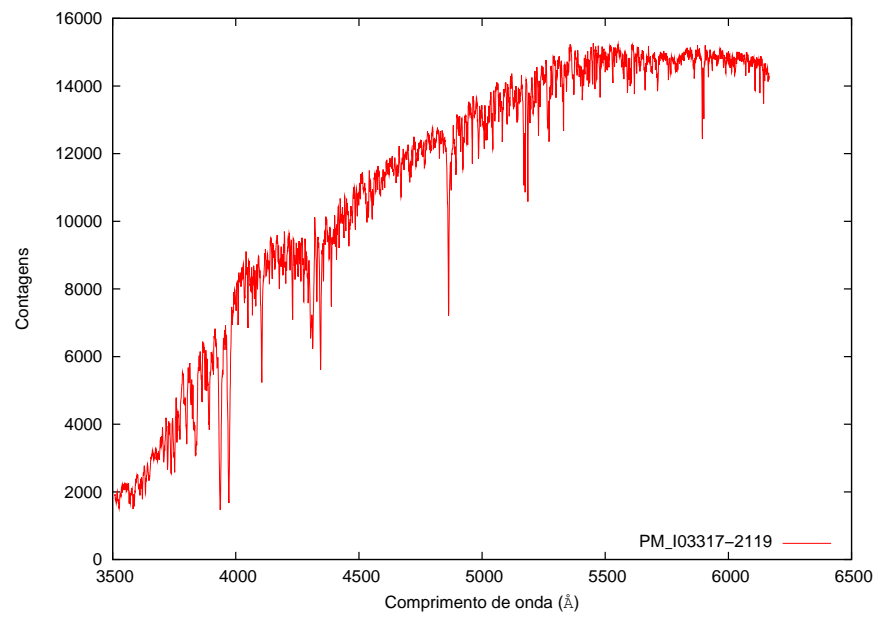

\section{Não observada}

Figura B.32: Par 37

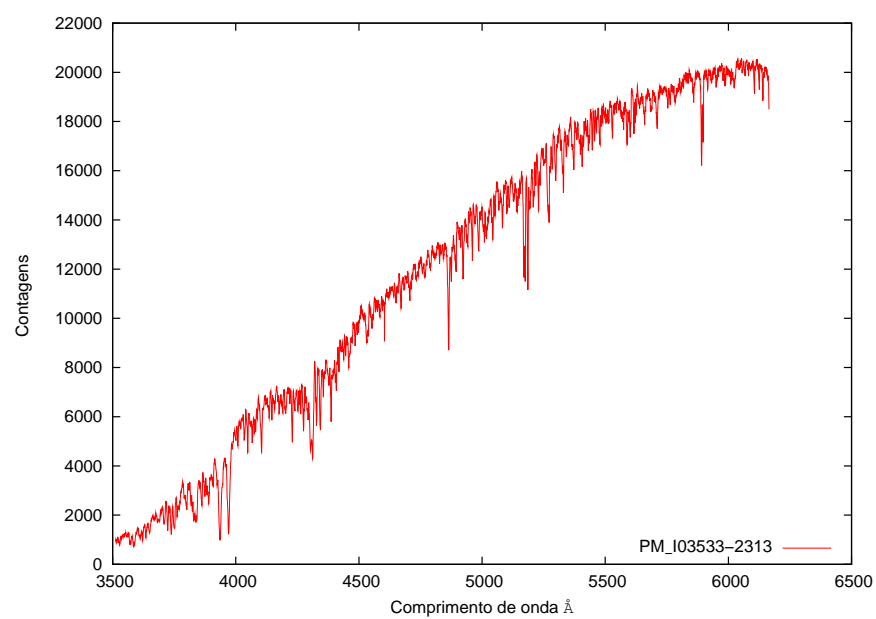

Não observada

Figura B.33: Par 38 


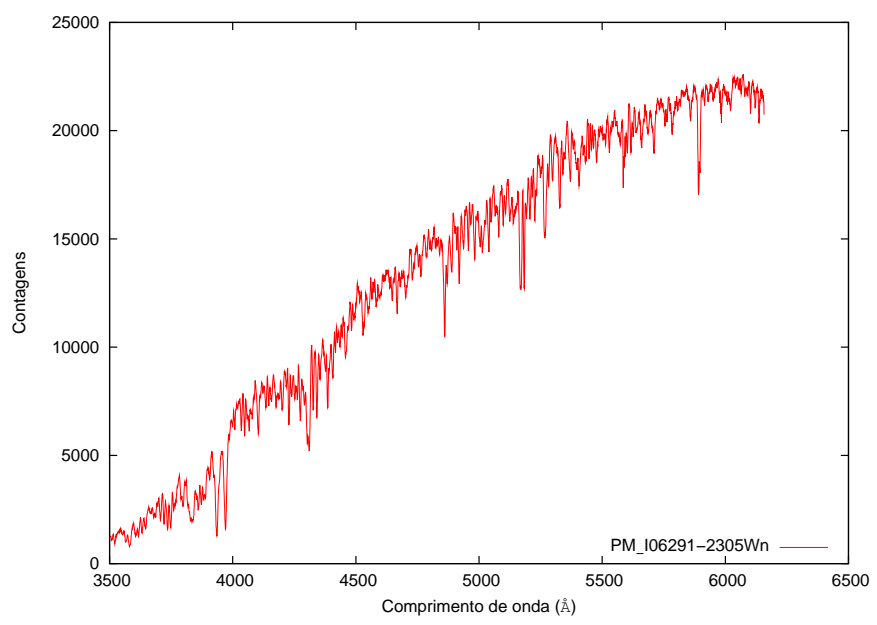

Não observada

Figura B.34: Par 39

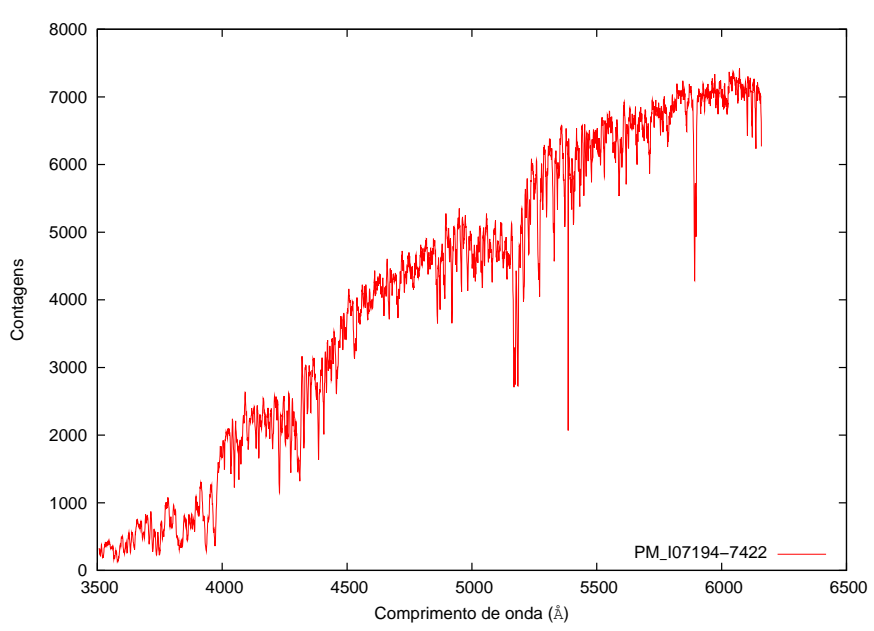

Não observada

Figura B.35: Par 40 